\title{
THE LICK AGN MONITORING PROJECT 2011: SPECTROSCOPIC CAMPAIGN AND EMISSION-LINE LIGHT CURVES
}

\author{
Aaron J. Barth ${ }^{1}$, Vardha N. Bennert ${ }^{2}$, Gabriela Canalizo ${ }^{3}$, Alexei V. Filippenko ${ }^{4}$, Elinor L. Gates ${ }^{5}$, Jenny E. Greene ${ }^{6}$, \\ Weidong Li $^{4,26}$, Matthew A. Malkan ${ }^{7}$, Anna Pancoast ${ }^{8}$, David J. Sand ${ }^{9}$, Daniel Stern ${ }^{10}$, Tommaso Treu ${ }^{7}$, \\ Jong-HaK Woo $^{11}$, Roberto J. Assef ${ }^{12}$, Hyun-Jin Bae ${ }^{13}$, Brendon J. Brewer ${ }^{14}$, S. Bradley Cenko ${ }^{15,16}$, Kelsey I. Clubb ${ }^{4}$, \\ Michael C. Cooper $^{1}$, Aleksandar M. Diamond-Stanic ${ }^{17}$, Kyle D. Hiner ${ }^{18}$, Sebastian F. Hönig ${ }^{19}$, Eric Hsiao ${ }^{20}$, \\ Michael T. KandrashofF ${ }^{4}$, Mariana S. Lazarova ${ }^{21}$, A. M. Nierenberg ${ }^{22}$, Jacob Rex ${ }^{4}$, JeFFrey M. Silverman ${ }^{23}$, \\ ERIK J. TOLLERUd 24,27 , AND JONELle L. WALSH ${ }^{25}$ \\ ${ }^{1}$ Department of Physics and Astronomy, 4129 Frederick Reines Hall, University of California, Irvine, CA, 92697-4575, USA; barth@uci.edu \\ ${ }^{2}$ Physics Department, California Polytechnic State University, San Luis Obispo, CA 93407, USA \\ 3 Department of Physics and Astronomy, University of California, Riverside, CA 92521, USA \\ Department of Astronomy, University of California, Berkeley, CA 94720-3411, USA \\ ${ }^{5}$ Lick Observatory, P.O. Box 85, Mount Hamilton, CA 95140, USA \\ ${ }^{6}$ Department of Astrophysical Sciences, Princeton University, Princeton, NJ 08544, USA \\ ${ }^{7}$ Department of Physics and Astronomy, University of California, Los Angeles, CA 90095-1547, USA \\ ${ }^{8}$ Department of Physics, University of California, Santa Barbara, CA 93106, USA \\ ${ }^{9}$ Texas Tech University, Physics Department, Box 41051, Lubbock, TX 79409-1051, USA \\ ${ }^{10}$ Jet Propulsion Laboratory, California Institute of Technology, 4800 Oak Grove Boulevard, Pasadena, CA 91109, USA \\ ${ }^{11}$ Astronomy Program, Department of Physics and Astronomy, Seoul National University, Seoul 151-742, Korea
${ }^{12}$ Núcleo de Astronomía de la Facultad de Ingeniería, Universidad Diego Portales, Av. Ejército Libertador 441, Santiago, Chile \\ ${ }^{13}$ Department of Astronomy and Center for Galaxy Evolution Research, Yonsei University, Seoul 120-749, Korea \\ ${ }_{15}^{14}$ Department of Statistics, The University of Auckland, Private Bag 92019, Auckland 1142, New Zealand \\ 15 Astrophysics Science Division, NASA Goddard Space Flight Center, MC 661, Greenbelt, MD 20771, USA \\ 16 Joint Space-Science Institute, University of Maryland, College Park, MD 20742, USA \\ ${ }^{17}$ Department of Astronomy, University of Wisconsin, Madison, WI 53706, USA \\ ${ }^{18}$ FONDECYT Postdoctoral Fellow, Departamento de Astronomía, Universidad de Concepción, Chile \\ ${ }^{19}$ School of Physics \& Astronomy, University of Southampton, Southampton SO17 1BJ, UK \\ 20 Department of Physics and Astronomy, Aarhus University, Ny Munkegade, DK-8000 Aarhus C, Denmark
${ }_{21}$ \\ ${ }^{21}$ Dept.of Physics and Physical Science, University of Nebraska at Kearney, Kearney, NE 68849, USA \\ ${ }^{22}$ Center for Cosmology and Astro-Particle Physics, The Ohio State University, Columbus OH 43210, USA \\ ${ }^{23}$ Department of Astronomy, The University of Texas, Austin, TX 78712-0259, USA \\ ${ }^{24}$ Astronomy Department, Yale University, New Haven, CT 06510, USA \\ ${ }^{25}$ George P. and Cynthia Woods Mitchell Institute for Fundamental Physics and Astronomy, Department of Physics and Astronomy, \\ Texas A\&M University, College Station, TX 77843-4242, USA \\ Received 2014 December 31; accepted 2015 February 24; published 2015 April 15
}

\begin{abstract}
In the Spring of 2011 we carried out a 2.5 month reverberation mapping campaign using the $3 \mathrm{~m}$ Shane telescope at Lick Observatory, monitoring 15 low-redshift Seyfert 1 galaxies. This paper describes the observations, reductions and measurements, and data products from the spectroscopic campaign. The reduced spectra were fitted with a multicomponent model in order to isolate the contributions of various continuum and emission-line components. We present light curves of broad emission lines and the active galactic nucleus (AGN) continuum, and measurements of the broad $\mathrm{H} \beta$ line widths in mean and rms spectra. For the most highly variable AGNs we also measured broad $\mathrm{H} \beta$ line widths and velocity centroids from the nightly spectra. In four AGNs exhibiting the highest variability amplitudes, we detect anticorrelations between broad $\mathrm{H} \beta$ width and luminosity, demonstrating that the broad-line region "breathes" on short timescales of days to weeks in response to continuum variations. We also find that broad $\mathrm{H} \beta$ velocity centroids can undergo substantial changes in response to continuum variations; in NGC 4593 , the broad $\mathrm{H} \beta$ velocity shifted by $\sim 250 \mathrm{~km} \mathrm{~s}^{-1}$ over a 1 month period. This reverberation-induced velocity shift effect is likely to contribute a significant source of confusion noise to binary black hole searches that use multi-epoch quasar spectroscopy to detect binary orbital motion. We also present results from simulations that examine biases that can occur in measurement of broad-line widths from rms spectra due to the contributions of continuum variations and photon-counting noise.
\end{abstract}

Key words: galaxies: active - galaxies: nuclei - galaxies: Seyfert - techniques: spectroscopic

Supporting material: machine-readable table

\section{INTRODUCTION}

Broad emission lines are a hallmark property of active galactic nuclei (AGNs), first highlighted in pioneering work by

\footnotetext{
26 Deceased 2011 December 12.

27 Hubble Fellow.
}

Seyfert (1943). Originating from the broad-line region (BLR), they serve as important diagnostics of physical conditions within the AGN as well as dynamical tracers providing information about the mass of the supermassive black hole at the core of the AGN central engine. Unfortunately, even for the nearest AGNs the BLR is too small to be directly resolved by any current observational capability. For example, in the well- 
studied nearby Seyfert 1 galaxy NGC 5548, the radius of the $\mathrm{H} \beta$-emitting portion of the BLR is $\sim 4-20$ light-days (Bentz et al. 2007), corresponding to an angular radius of just 9-45 microarcseconds.

Although the spatial structure of the BLR cannot be directly resolved, the time-variable nature of AGN emission makes it possible to resolve the BLR's size and structure in the time domain via reverberation mapping (Blandford \& McKee 1982; Peterson 2001). Ionizing photons from the AGN's central engine are reprocessed by BLR gas into emission-line photons. From the vantage point of a distant observer, random variations in the flux of the AGN continuum will be followed by corresponding variations in the fluxes of emission lines from the BLR, with a time delay that depends on the light-travel time across the BLR. Cross-correlation of emission-line light curves against light curves of the AGN continuum gives a measure of the BLR radius $r_{\mathrm{BLR}}$, under the assumption that the size of the continuum emission region is much smaller than $r_{\mathrm{BLR}}$. Reverberation mapping thus requires measurement of light curves of the variable AGN continuum and emission lines, with a cadence sufficient to resolve the temporal fluctuations adequately. When $r_{\mathrm{BLR}}$ is combined with a velocity parameter measured from the width of the broad line, a so-called virial mass estimate can be derived for the central black hole. Virial masses have been determined through reverberation mapping for several dozen low-redshift AGNs, and these measurements form the foundation of secondary "single-epoch" virial mass methods, which provide nearly all of the empirical information currently available on the cosmological growth history and redshift evolution of supermassive black holes (for a comprehensive review, see Shen 2013).

A major goal for reverberation mapping observations is the detection of velocity-resolved emission-line variations- that is, variations in the shapes of broad emission-line profiles, not merely variations in total emission-line flux. Line profile variability conveys information about the kinematics of BLR gas. Measurements of profile variability can provide a means to discriminate between rotationally dominated, inflowing, or outflowing BLR kinematics (e.g., Welsh \& Horne 1991), while high-cadence and high signal-to-noise ratio $(\mathrm{S} / \mathrm{N})$ observations can potentially reveal a wealth of detail about BLR structure and kinematics (Horne et al. 2004).

It is a nontrivial challenge to obtain observations with sufficiently high cadence and high $\mathrm{S} / \mathrm{N}$ suitable for measurement of broad-line profile variations. Consequently, many of the largest reverberation-mapping programs have focused largely on measurement of flux variations and derivation of black hole virial masses (e.g., Kaspi et al. 2000; Peterson et al. 2004, and references therein). Over the past decade or so, various observing programs have succeeded in measuring velocity-resolved variability signatures in broad $\mathrm{H} \beta$; an early example was a sparsely sampled program at the Hobby-Eberly telescope targeting Mrk 110 (Kollatschny 2003). More recently, densely sampled observing campaigns have led to rapid progress. Using data from the 2008 Lick AGN Monitoring Project campaign, a 64-night program carried out at the Lick $3 \mathrm{~m}$ telescope, Bentz et al. $(2008,2009 \mathrm{~b})$ detected a strong gradient in the reverberation lag across the $\mathrm{H} \beta$ line profile in Arp 151 (Mrk 40), and the campaign provided some degree of velocity resolution of the $\mathrm{H} \beta$ reverberation lag in five additional AGNs. Similarly, intensive monitoring campaigns carried out at the MDM Observatory have recently yielded impressive detections of velocity-resolved reverberation (Denney et al. 2009b; Grier et al. 2013a). The collective results of these programs suggest a surprising diversity in the kinematic properties of AGNs, with different objects exhibiting behavior consistent with rotation, infall, or outflow as the dominant kinematic behavior in the BLR.

These high-cadence monitoring programs at Lick and MDM have spurred the development of new methods for analysis of reverberation data. The traditional approach of deriving black hole masses by applying a simple recipe to derive the "virial product" $\left[\mathrm{VP}=r_{\mathrm{BLR}}(\Delta V)^{2} / G\right.$, where $r_{\mathrm{BLR}}$ is determined from the reverberation lag and $\Delta V$ is a measure of the broad-line width] suffers from several shortcomings. In particular, the "BLR radius" measured from the reverberation lag gives a highly oversimplified measure of BLR structure, since the BLR actually spans a very broad range in radius. Similarly, a single measure of BLR velocity derived from an emission-line width is a gross oversimplification of the complex velocity structure of a real BLR. More worrying is the possibility that the BLR dynamics might be affected by outflows or other nongravitational motions, in which case simple virial mass estimates might be entirely misleading (Krolik 2001). Pancoast et al. (2011) proposed a different approach to reverberation data analysis, in which black hole masses can be determined via a forward-modeling procedure that computes the response to continuum variations of a physically motivated dynamical model for BLR clouds. By exploring a broad parameter space of BLR dynamical models and comparing the model predictions directly with the observed time series of broad emissionline profiles, direct constraints can be placed on the black hole mass, BLR inclination and opening angle, radial emissivity profile, and other parameters. This approach has been applied to several AGNs from our high-cadence Lick programs (Brewer et al. 2011; Pancoast et al. 2012, 2014b). Li et al. (2013) presented applications of an independent implementation of a similar method to archival data from several observing campaigns.

Although reverberation mapping has seen dramatic progress over the past several years stemming from these high-cadence monitoring campaigns, there are still only a handful of AGNs having time-series data with sufficient sampling and $\mathrm{S} / \mathrm{N}$ for these dynamical modeling methods to be optimally successful. In order to obtain more velocity-resolved reverberation data suitable for dynamical modeling, we embarked on a new reverberation mapping program in the spring of 2011. Spectroscopic observations were conducted at the Lick $3 \mathrm{~m}$ Shane telescope, and $V$-band photometric monitoring was carried out using several queue-scheduled and robotic telescopes. This paper describes the spectroscopic campaign and presents measurements from the Lick data.

A new aspect of our data analysis for this 2011 program is the use of spectral fitting and decomposition techniques to isolate different portions of the variable spectra. In the past, nearly all emission-line light curves for reverberation mapping were measured by subtracting a local, linear continuum model underlying strong emission lines such as $\mathrm{H} \beta$ in order to isolate the emission-line flux. This method is generally effective for measuring integrated light curves of strong emission lines having high variability amplitude. However, simple linear continuum subtraction is unable to remove host-galaxy starlight features that can affect the broad-line profiles and widths, and it cannot disentangle blended emission lines. The alternative 
approach of fitting models to deblend components such as Fe II from $\mathrm{H} \beta$ had seldom been applied to nightly spectra from reverberation campaigns (e.g., Bian et al. 2010). Our initial experiments with spectral decomposition using observations of Mrk 50 from this program established that multicomponent fits made it possible to measure light curves for He II $\lambda 4686$ even when the line was heavily diluted by starlight, and to detect variability and measure reverberation lags of the optical Fe II blends (Barth et al. 2011b, 2013). Using a similar fitting method, Park et al. (2012a) carried out decompositions of spectra from the 2008 Lick AGN Monitoring Project campaign (Bentz et al. 2009b). This work demonstrated clear improvements in determination of the mean and rms line profiles for $\mathrm{H} \beta$ after removal of the host-galaxy starlight and other blended line components from the spectra. In this paper, we present a complete description of our fitting method and results from applying the spectroscopic decomposition procedure to our full 2011 monitoring sample.

The outline of this paper is as follows. Section 2 describes the sample selection, and Section 3 describes the spectroscopic observations carried out at Lick. Details of the data reduction, calibrations, and the fitting procedure applied to the blue-side spectra are given in Section 4. Sections 5 and 6 present the measurements of emission-line light curves, broad $\mathrm{H} \beta$ line widths, and velocity centroid variations. Section 7 examines the anticorrelation between broad $\mathrm{H} \beta$ width and luminosity in the single-epoch spectra of highly variable sources. In Section 8 we describe how broad-line velocity shifts induced by asymmetric emission-line reverberation can potentially mimic the observational signature of orbital motion in a binary black hole system, and we consider the implications of broad-line reverberation for spectroscopic binary black hole searches. Section 9 presents a summary of our results and conclusions. In Appendix A, we display spectra of additional AGNs that were observed during the first few nights of our program as we finalized our target list for monitoring. Appendices $\mathrm{B}$ and $\mathrm{C}$ present simulations designed to clarify the relationship between line widths measured from mean and rms spectra, and to demonstrate potential sources of bias that can afflict broad-line widths measured from rms spectra.

\section{SAMPLE SELECTION}

The major science goal for this program was to measure velocity-resolved reverberation signals in $\mathrm{H} \beta$ for bright Seyfert 1 galaxies, and we primarily selected targets having strong broad $\mathrm{H} \beta$ emission from the Sloan Digital Sky Survey (SDSS) Data Release 7 archive (Abazajian et al. 2009) and other AGN catalogs in the literature. Our reverberation campaign began in late March and continued through the middle of June. This set a requirement for the sample to contain AGNs distributed over a broad range of right ascension (R.A.) such that the entire sample could be observed over nearly the full duration of the program. Targets were selected to have apparent magnitudes of $V \lesssim 17$ so that high-S/N spectra could be obtained in short exposures (10-40 minutes). We chose AGNs for which estimated $\mathrm{H} \beta$ reverberation lags were $<25$ days based on either the radius-luminosity relation from Bentz et al. (2009a) or previous reverberation measurements. Finally, the standard configuration of the Kast spectrograph employs a 600-line grism in the blue camera and a dichroic with cutoff wavelength $5500 \AA$, and the blue-camera throughput drops steeply at $\lambda>5400 \AA$. This imposes a practical redshift limit of $z \lesssim 0.08$ in order for $[\mathrm{O} \mathrm{III}] \lambda 5007$ to fall on the high-sensitivity portion of the blue camera. This line is used as the reference for flux calibration of the spectral time series.

Two targets, Mrk 817 and Zw 229-015, were included in the sample in order to coordinate with a Spitzer infrared monitoring program taking place in 2011 (Spitzer program ID 70119; PI V. Gorjian). Zw 229-015 was also a Kepler monitoring target during this time (Mushotzky et al. 2011; Carini \& Ryle 2012; Edelson et al. 2014).

We assembled an initial set of 15 high-priority targets plus several alternates which included narrow-line Seyfert 1 galaxies, AGNs that were somewhat fainter than our primary targets, and candidate AGNs for which no high-quality recent spectra were available in the literature. During the first few usable nights of the campaign, we found that three of our toppriority targets selected from SDSS had transformed into Type 1.9 Seyferts with essentially no broad $\mathrm{H} \beta$ emission remaining (see Appendix A for details). Several alternate targets were then observed and we chose the most promising ones to fill the gaps in the sample's R.A. range. Our final monitoring sample is listed in Table 1, and Appendix A presents spectra of the alternate and discarded targets.

Some of these AGNs have been observed in previous reverberation programs, including Mrk 40 (Arp 151; Bentz et al. 2008, 2009b), Mrk 279 (Maoz et al. 1990; Santos-Lleó et al. 2001), Mrk 817 (Peterson et al. 1998; Denney et al. 2010), NGC 4593 (Dietrich et al. 1994; Onken et al. 2003; Denney et al. 2006), and Zw 229-015 (Barth et al. 2011a). Revised lag measurements of Mrk 279, Mrk 817, and NGC 4593 were also given in the compilation of Peterson et al. (2004). Recently, Wang et al. (2014) presented results from a reverberation campaign that included Mrk 486 and Mrk 493 as well. New observations of previously well-observed targets such as Mrk 40 can potentially test whether velocity-resolved reverberation lags remain constant or vary over durations comparable to the dynamical timescale of the BLR.

Owing to the combination of criteria given above, the observed sample is not complete or unbiased in terms of black hole mass, luminosity, broad-line width, or any other fundamental or observed property. This is a recurring issue for all AGN reverberation mapping campaigns to date, and should be kept in mind when extrapolating reverberation results to derive single-epoch masses for AGNs at high luminosities or Eddington ratios not probed by the reverberation-mapped sample. This point has been emphasized by Richards et al. (2011) and Shen (2013) in the context of examining biases inherent in single-epoch masses derived from the $\mathrm{C}$ IV line.

\section{OBSERVATIONS}

This project was allocated 69 nights at the Lick $3 \mathrm{~m}$ Shane telescope, distributed between 2011 March 27 and June 13 (all dates are UT). Interspersed with our nights were occasional gaps, mostly during or close to full moon, for other projects such as exoplanet search programs.

Observations were conducted using the Kast double spectrograph (Miller \& Stone 1993). On the blue side (spatial scale 0.43 pixel $^{-1}$ ), we used a 600 lines $\mathrm{mm}^{-1}$ grism covering 3440-5515 $\AA$ at $1.02 \AA$ pixel $^{-1}$. On the red side (spatial scale 0 .78 pixel $^{-1}$ ), we used a 600 lines $\mathrm{mm}^{-1}$ grating blazed at $7500 \AA$, giving coverage of $5410-8200 \AA$ at $2.35 \AA$ pixel ${ }^{-1}$. 
Table 1

Sample Properties and Observation Parameters

\begin{tabular}{|c|c|c|c|c|c|c|c|c|c|}
\hline Galaxy & Alt. Name & $z$ & $\begin{array}{c}D_{L} \\
(\mathrm{Mpc})\end{array}$ & $\begin{array}{c}A_{V} \\
(\mathrm{mag})\end{array}$ & $\begin{array}{l}\text { Slit PA } \\
\text { (deg) }\end{array}$ & $N_{\text {obs }}$ & $\begin{array}{l}\text { Mean Sampling } \\
\text { (days) }\end{array}$ & $\begin{array}{l}\text { Median } \\
\text { Airmass }\end{array}$ & $\begin{array}{c}\text { Median } \\
\text { S/N }\end{array}$ \\
\hline Mrk 40 & Arp 151 & 0.0211 & 92.3 & 0.039 & 90 & 39 & 1.97 & 1.07 & 72 \\
\hline Mrk 50 & $\ldots$ & 0.0234 & 102.5 & 0.044 & 180 & $55^{\mathrm{a}}$ & $1.81^{\mathrm{c}}$ & 1.29 & 77 \\
\hline Mrk 141 & $\ldots$ & 0.0417 & 185.2 & 0.028 & 90 & 36 & 1.79 & 1.16 & 107 \\
\hline Mrk 279 & PG 1351+695 & 0.0305 & 134.3 & 0.044 & 120 & 34 & 2.30 & 1.19 & 106 \\
\hline Mrk 486 & PG $1535+547$ & 0.0389 & 172.4 & 0.040 & 90 & 27 & 2.28 & 1.08 & 94 \\
\hline Mrk 493 & $\cdots$ & 0.0313 & 137.9 & 0.068 & 70 & 32 & 2.39 & 1.08 & 88 \\
\hline Mrk 504 & PG 1659+294 & 0.0359 & 158.7 & 0.135 & 60 & 36 & 2.18 & 1.05 & 89 \\
\hline Mrk 704 & $\cdots$ & 0.0292 & 128.5 & 0.079 & 45 & 38 & 1.68 & 1.15 & 70 \\
\hline Mrk 817 & PG $1434+590$ & 0.0315 & 138.8 & 0.019 & 90 & 27 & 2.42 & 1.08 & 84 \\
\hline Mrk 841 & PG $1501+106$ & 0.0364 & 161.0 & 0.082 & 45 & 35 & 1.82 & 1.14 & 94 \\
\hline Mrk 1392 & $\ldots$ & 0.0361 & 160.1 & 0.125 & 45 & 38 & 2.02 & 1.24 & 98 \\
\hline Mrk 1511 & NGC 5940 & 0.0339 & 149.7 & 0.112 & 45 & 40 & 2.00 & 1.21 & 72 \\
\hline NGC 4593 & Mrk 1330 & 0.0090 & 39.0 & 0.068 & 45 & 43 & 1.85 & 1.44 & 121 \\
\hline PG 1310-108 & II SZ 10 & 0.0343 & 151.5 & 0.143 & 180 & 35 & 2.30 & 1.58 & 50 \\
\hline Zw 229-015 & $\ldots$ & 0.0279 & 122.6 & 0.198 & 56.5 & $29^{\mathrm{b}}$ & $2.72^{\mathrm{c}}$ & 1.07 & 49 \\
\hline
\end{tabular}

Note. Redshifts are taken from NED. Luminosity distances $\left(D_{L}\right)$ are calculated from redshifts assuming a WMAP9 cosmology with $H_{0}=69.7 \mathrm{~km} \mathrm{~s}^{-1} \mathrm{Mpc}^{-1}$, $\Omega_{M}=0.281$, and $\Omega_{\Lambda}=0.7185$ (Hinshaw et al. 2013), using the Wright (2006) calculator. Galactic extinctions $\left(A_{V}\right)$ are from NED and are based on Schlafly \& Finkbeiner (2011), assuming $R_{V}=3.1 . N_{\mathrm{obs}}$ is the total number of spectroscopic observations of each AGN. Mean sampling is the mean time interval between successive observations, in days. (The median sampling interval is 1.0 days for all AGNs.) The median S/N gives the median S/N per pixel between rest wavelengths 4600 and $4700 \AA$ in the extracted blue-side spectra, for the full series of observations of each AGN.

${ }^{a}$ Twelve of the 55 spectra of Mrk 50 were obtained before the start of our main campaign during nights assigned to other projects.

${ }^{b}$ Three of the 29 spectra of $Z w 229-015$ were obtained after the end of our main campaign during nights assigned to other projects.

${ }^{\mathrm{c}}$ Mean sampling for Mrk 50 and Zw 229-015 was calculated based on observations taken during the main campaign.

The d55 dichroic was employed to split the light between the blue and red cameras. ${ }^{28} \mathrm{~A}$ slit width of $4^{\prime \prime}$ was adopted in order to mitigate slit losses while also preserving moderate spectral resolution. Calibration frames taken each afternoon included bias exposures, dome flats, and wavelength-calibration lamps including $\mathrm{He}, \mathrm{Ne}, \mathrm{Ar}, \mathrm{Hg}$, and $\mathrm{Cd}$. The red-side $\mathrm{CCD}$ exhibits a steep change in bias level at the short-wavelength end, while the blue-side detector has extremely clean and flat bias structure with no discernible persistent features.

The red-side spectra are affected by strong fringing at $\lambda \gtrsim 7000 \AA$, but it can be removed fairly well by flat-field division if a dome-flat exposure is taken at the same telescope position and spectrograph rotation angle as the object. While the dome-flat exposures are very short (1-2s), this requires additional overhead time for the dome to be rotated or even capped (if the target is close to the zenith). In order to maximize the on-source exposure time during the nights, we opted to use only afternoon dome-flat exposures rather than observing dome flats at the position of each AGN during the nights. This trade-off compromises the data quality of the redside exposures at wavelengths longer than $7000 \AA$, but it does not affect the blue-side data which are the main focus of our reverberation measurements.

Exposure times for each AGN were between 300 and 2400 s and were adjusted during the campaign as the nights grew shorter. Longer integrations were usually split into two individual exposures to facilitate cosmic-ray rejection.

Each AGN was observed at a fixed slit position angle (PA) throughout the program in order to maintain consistency in the amount of spatially extended host-galaxy emission within the slit (Table 1). Additionally, the flux-scaling method applied to

\footnotetext{
28 In our 2008 Lick campaign (Bentz et al. 2009b), we used only the red arm of the Kast spectrograph because the blue-side CCD had recently failed and was replaced at the time with a temporary, lower-efficiency detector.
}

the spectra relies on the assumption of a constant [O III] flux, and observing at a fixed PA ensures consistency in the portion of the narrow-line region subtended by the slit, which can be important for low-redshift AGNs having spatially extended emission. The PA for each object was chosen to be close to the parallactic angle (Filippenko 1982) during the portion of the campaign when the object would be observed at relatively higher airmass. Since the observations were not carried out with the slit at the parallactic angle, wavelength-dependent slit losses have an impact on the quality of the relative flux calibration, although those losses are somewhat mitigated by the use of the 4 "-wide slit. Slit losses contribute spurious scatter to the continuum light curves measured from the blue end of the spectra and to the $\mathrm{H} \alpha$ light curves measured from the redside data. For Zw 229-015, the slit PA was set to 56:5 in order to include a foreground star in the slit and for consistency with previous measurements (Barth et al. 2011a).

On clear nights we attempted to observe each AGN in the sample, but Zw 229-015 (at R.A. 19 hr) was only occasionally observed during the first month of the program. As the campaign progressed and nights became shorter, exposure times were shortened for most objects. We also ended monitoring for Mrk 141, Mrk 486, Mrk 817, and Mrk 841 about two weeks before the end of the campaign in order to conserve observing time for higher-priority targets. The weather at Lick was worse than average during the spring of 2011. Of the 69 nights, 20 were entirely lost to bad weather, and another 12 nights suffered $\gtrsim 50 \%$ losses of observing time to clouds or high humidity. Only 23 nights were fully usable with no significant weather-related losses, while the remaining 14 nights were mildly to moderately impacted by clouds. Consequently, the number of observations per AGN was relatively small, and for most targets we obtained between 30 and 40 observations during the 69-night program. Table 1 lists the number of exposures, median airmass, and median $\mathrm{S} / \mathrm{N}$ at 
$4600-4700 \AA$ for each object. During partially usable nights, the objects observed at highest priority included Mrk 40, Mrk 50, Mrk 1511, and NGC 4593.

In order to extend our light curves for two AGNs, we also requested additional observations from other observers using the Kast spectrograph: Mrk 50 from 2011 January through March, and Zw 229-015 in June and July. The Mrk 50 data (including observations by other observers) were previously presented by Barth et al. (2011b). Unfortunately, the 12 observations taken prior to the start of our campaign suffered from large sampling gaps owing to winter weather, and do not add much additional information to the lag measurements. For Zw 229-015, three additional observations were taken 20-23 days after the end of our main campaign.

During each night, time was also set aside for one spectroscopic observation of a bright supernova or other transient. Observations taken during our program have been included in publications examining the spectral evolution of $\mathrm{SN}$ 2010j1 (Smith et al. 2012), SN 2011ay (Foley et al. 2013), SN 2011 by (Silverman et al. 2013), and the young stellar object PTF 10nvg (Hillenbrand et al. 2013), and have been described in Central Bureau Electronic Telegrams (CBETs) 2681 (SN 2011ay), 2699 (SN 2011bp), 2701 (SN 2011bq), 2702 (SN $2011 \mathrm{br}$ ), 2712 (SN 2011cc), 2716 (SN 2011cf), 2721 (SN $2011 \mathrm{cj})$, and 2752 (SN 2011dt). ${ }^{29}$

\section{DATA PROCESSING}

\subsection{Spectroscopic Data Reduction}

The initial data reduction followed standard procedures including overscan subtraction, two-dimensional bias subtraction (for the red-side CCD only), flat-fielding, and cosmic-ray cleaning using the LA-Cosmic routine (van Dokkum 2001) implemented as an $\operatorname{IRAF}^{30}$ script. The IRAF response task was used to produce normalized flat-field frames having pixel values close to unity. Spectral extractions were done with the IRAF apall task. An unweighted extraction of width 10'3 was adopted in order to minimize the impact of nightly seeing variations. The wavelength scale was established by applying a polynomial fit to the line-lamp spectra observed each afternoon, and then applied to the extracted spectra. For each wavelengthcalibrated spectrum, a small final linear shift to the wavelength scale was applied based on offsets measured from strong emission lines in the corresponding night-sky spectrum. Flux calibration and removal of telluric absorption features (following the procedure of Wade \& Horne 1988) were carried out using IDL routines as described by Matheson et al. (2000). The calibrated spectra were rebinned to a linear wavelength scale of $1.0 \AA \mathrm{pixel}^{-1}$ on the blue side and $2.0 \AA \mathrm{pixel}^{-1}$ on the red side. When two exposures of an AGN were taken on the same night, they were combined to produce a final weighted average spectrum.

Error spectra produced by the apall task were propagated through all steps of the calibration pipeline. For spectra of these spatially extended AGNs, it is necessary to use the unweighted extractions because optimal extractions (Horne 1986) have a tendency to truncate the peaks of strong emission lines such as

\footnotetext{
29 CBETs are distributed via http://www.cbat.eps.harvard.edu/index.html.

30 IRAF is distributed by the National Optical Astronomy Observatories, which are operated by the Association of Universities for Research in Astronomy, Inc., under cooperative agreement with the National Science Foundation.
}

[O III] when they have a different spatial extent than the surrounding continuum flux. However, the apall error spectrum actually gives the pixel uncertainties (owing to photon counting and readout noise) on the optimally weighted extraction, not on the unweighted extraction. For our high-S/N observations (typically with $\mathrm{S} / \mathrm{N} \approx 50-100 \mathrm{pixel}^{-1}$ ), the differences between the unweighted and optimally weighted extractions are very small, and the error spectra will only slightly underestimate the actual photon-counting uncertainties. As described below, systematic uncertainties in flux calibration are often a major or dominant source of noise in the spectroscopic light curves. This additional source of error is not accounted for in the extracted error spectra and must be added separately to the error budget after the spectral rescaling procedure is applied.

\subsection{Photometric Scaling}

Conditions at Lick are rarely photometric during the spring season, and the flux-calibrated spectra do not share a consistent overall flux scale. The wavelength scales of the calibrated spectra also exhibit small offsets from night to night, caused by random miscentering of the AGN in the spectrograph slit. Before carrying out the emission-line measurements, the wavelength and flux scales of the spectra must be aligned.

The blue-side data were treated as follows for each AGN individually. First, wavelength shifts between the nightly spectra were measured using a cross-correlation procedure, sampling in steps of 0.1 pixel. The wavelength shifts determined from cross-correlation were removed from the wavelength vector of each spectrum, and the aligned spectra were then averaged to produce an initial mean reference spectrum.

In order to place the reference spectrum on an approximate absolute photometric scale, we examined the nightly observing logs and selected nights listed by the observers as being either photometric or very clear; these candidate clear nights numbered only 14 during the 69-night campaign. For each AGN, the $\left[\mathrm{O}_{\text {III }}\right] \lambda 5007$ flux was measured from each candidate clear night's spectrum by integrating the line flux above a straight-line fit to adjacent continuum regions on either side of the line. Then, the standard deviation of these line fluxes was calculated, and $>2 \sigma$ outliers were discarded from the list of candidate photometric observations. This process was then repeated until there were no remaining $2 \sigma$ outliers in $[\mathrm{O}$ III] flux, and the mean $[\mathrm{O}$ III] flux from the remaining spectra was taken to be the best estimate of the true line flux. The number of final "clear-night" spectra varied for each AGN and ranged between 6 and 9. Table 2 lists the $[\mathrm{O}$ III] fluxes based on this procedure; the quoted uncertainty in the table is the standard deviation of the fluxes measured on candidate photometric nights after excluding outliers. The reference spectrum was then multiplied by a scaling factor to normalize its $[\mathrm{O}$ III] flux to this photometric value. We caution that these $[\mathrm{O}$ III] fluxes should be considered only as estimates of the true fluxes, given the scarcity of truly photometric conditions at Lick.

Then, the procedure described by van Groningen \& Wanders (1992) was applied to the data to align the wavelength and flux scales under the assumption that the $[\mathrm{O}$ III] flux is intrinsically constant during the duration of the monitoring campaign. Long-duration monitoring of NGC 5548 confirms that this is a reasonable assumption, although $[\mathrm{O}$ III $]$ flux variations have been detected on timescales of years (Peterson et al. 2013). The 
Table 2

[O III] and Featureless Continuum Fluxes

\begin{tabular}{lccc}
\hline \hline Object & $f([\mathrm{O}$ III $])$ & $\sigma_{\mathrm{nx}}([\mathrm{O}$ III $])$ & $f_{\lambda}(5100 \AA)$ \\
\hline Mrk 40 & $68.8 \pm 6.2$ & 0.007 & $0.56 \pm 0.08$ \\
Mrk 50 & $16.3 \pm 2.1$ & 0.033 & $1.20 \pm 0.20$ \\
Mrk 141 & $34.1 \pm 3.7$ & 0.016 & $1.09 \pm 0.16$ \\
Mrk 279 & $157.6 \pm 7.7$ & 0.006 & $0.93 \pm 0.10$ \\
Mrk 486 & $65.4 \pm 4.3$ & 0.019 & $2.15 \pm 0.26$ \\
Mrk 493 & $25.8 \pm 1.6$ & 0.023 & $1.43 \pm 0.17$ \\
Mrk 504 & $17.3 \pm 0.5$ & 0.015 & $0.57 \pm 0.06$ \\
Mrk 704 & $114.7 \pm 14.5$ & 0.005 & $2.32 \pm 0.37$ \\
Mrk 817 & $143.7 \pm 18.7$ & 0.013 & $5.69 \pm 0.92$ \\
Mrk 841 & $270.4 \pm 19.1$ & 0.009 & $3.63 \pm 0.45$ \\
Mrk 1392 & $180.0 \pm 28.8$ & 0.006 & $2.41 \pm 0.45$ \\
Mrk 1511 & $31.3 \pm 0.5$ & 0.015 & $0.86 \pm 0.09$ \\
NGC 4593 & $144.4 \pm 5.8$ & 0.023 & $2.64 \pm 0.29$ \\
PG 1310-108 & $128.2 \pm 2.7$ & 0.007 & $1.66 \pm 0.17$ \\
Zw 229-015 & $37.4 \pm 1.3$ & 0.007 & $0.56 \pm 0.06$ \\
\hline
\end{tabular}

Note. [O $\mathrm{III}]$ line fluxes are given in the observed frame in units of $10^{-15} \mathrm{erg} \mathrm{cm}$ ${ }^{-2} \mathrm{~s}^{-1}$, and uncertainties represent the standard deviation of [O III] fluxes measured on candidate photometric nights. The quantity $\sigma_{\mathrm{nx}}$ is the normalized excess scatter in the [O III] light curve, as defined in Section 4.2. For Mrk 50, data points taken before the start of the main campaign were excluded from the computation of $\sigma_{\mathrm{nx}}$, because the earlier observations were taken with heterogeneous spectrograph setups and had a much larger flux scaling scatter. The AGN featureless continuum flux density is given in the AGN rest frame at $5100 \AA$ in units of $10^{-15} \mathrm{erg} \mathrm{cm}^{-2} \mathrm{~s}^{-1} \AA^{-1}$, and is measured from the powerlaw component of the mean spectrum decomposition and corrected for Galactic extinction.

van Groningen \& Wanders (1992) procedure applies a scaling factor, a linear wavelength shift, and a Gaussian broadening to the individual night's spectrum, and finds the parameter values which minimize the value of $\chi^{2}$ when fitting a low-order polynomial to the difference between the reference spectrum and the adjusted individual spectrum, over a small wavelength range including $\left[\mathrm{O}_{\mathrm{III}}\right]$ and some redward continuum. Since the spectral focus and line-spread function can vary from night to night, and either the individual spectrum or the reference spectrum might have a narrower line-spread function, the procedure is repeated with the Gaussian broadening kernel applied to the reference spectrum instead of the individual spectrum, and the final scaled spectrum is taken to be the better of the two options in terms of the $\chi^{2}$ value obtained. Instead of using a grid search to find the best-fit values of the three free parameters as described by van Groningen \& Wanders (1992), our method uses downhill simplex optimization, implemented with the IDL amoeba procedure, to minimize $\chi^{2}$. We found that over the full time series of spectra for a given AGN, the shifts in wavelength scale needed to align the spectra were at the level of $\sim 1 \AA$ rms. The final blue-side spectra are rebinned to a uniform wavelength scale of $1.0 \AA$ pixel $^{-1}$ with integer wavelength values in $\AA$.

To test the effectiveness of the spectral scaling, we can examine the scatter in $[\mathrm{O}$ III $]$ flux values for each AGN over the course of the campaign. Since the scaling procedure essentially matches the $\left[\mathrm{O}_{\mathrm{III}}\right]$ profile for each nightly spectrum to the reference spectrum, but does not explicitly force all [O III] fluxes to be identical, the dispersion among [O III] fluxes for a given AGN gives a measure of the final random uncertainties in the flux calibration after the scaling procedure has been applied. We use the normalized excess variance $\sigma_{\mathrm{nx}}^{2}$ to quantify the light-curve variance over and above the amount expected from the propagated measurement uncertainties:

$$
\sigma_{\mathrm{nx}}^{2}=\frac{1}{N\langle f\rangle^{2}} \sum_{i=1}^{N}\left[\left(f_{i}-\langle f\rangle\right)^{2}-\delta_{i}^{2}\right],
$$

where $N$ is the number of observations in the light curve, $\langle f\rangle$ is the mean flux, and $f_{i}$ and $\delta_{i}$ are the individual flux measurements and their uncertainties from photon counting and detector read noise. ${ }^{31}$ Then, $\sigma_{\mathrm{nx}}$ is a measure of the fractional rms scatter in the light curve over and above the amount expected from the propagated uncertainties; we refer to this quantity as the "normalized excess scatter." We measured $\sigma_{\mathrm{nx}}$ from the $[\mathrm{O} \mathrm{III}]$ light curves for each AGN, and the values are listed in Table 2. The values of $\sigma_{\mathrm{nx}}$ range from 0.005 to 0.033 (i.e., the excess scatter in the light curves ranges from 0.5 to $3.3 \%$ of the mean [O III] fluxes). The objects with the highest [O III] photometric scatter tend to be AGNs having low [O III] equivalent width, such as Mrk 50 and Mrk 493, for which the van Groningen \& Wanders (1992) scaling method does not work optimally.

We interpret $\sigma_{\mathrm{nx}}$ as a measure of the level of random error in the final flux normalization of the spectra. For measurements of reverberation lags or other quantities from the light curve, this source of error can be incorporated into the measurement error budget by adding $\sigma_{\mathrm{nx}} \times\langle f\rangle$ in quadrature to the photoncounting uncertainties on each light-curve point $f$. Since the spectral scaling is optimized for the wavelength of the [O III] $\lambda 5007$ line, the relative scaling at much longer or shorter wavelengths is expected to be worse than the amount indicated by $\sigma_{\mathrm{nx}}$, as a result of atmospheric dispersion and wavelengthdependent slit losses.

After applying the scaling procedure, mean and rms spectra are constructed following Peterson et al. (2004). The mean spectrum is a simple average of the individual nightly spectra. The rms spectrum is calculated by taking the standard deviation of flux values at each pixel over the time series, and gives an illustration of the relative variability amplitude of different spectral regions. Other prescriptions for calculating rms spectra have been explored by Park et al. (2012a). Since the rms spectrum includes the contribution of photon-counting noise and other sources of noise or random error in the data, in addition to genuine AGN variability, the amplitude of the rms spectrum is higher than it would be for the ideal case of noisefree data. Simulations presented in Appendix C demonstrate that photon-counting noise will bias the widths of broad lines in rms spectra to values that are typically several percent lower than they would be in the absence of noise.

The red-side data were generally flux-calibrated using standard-star observations obtained simultaneously with the blue-side standard-star observations. In the reduced data, the region of wavelength overlap between the blue-side and redside spectra is $5420-5500 \AA$. For each AGN observation, we normalized the red-side flux scale by applying a scaling factor to match it to the scaled blue-side spectrum over this wavelength range, and then produced mean and rms spectra from these rescaled red-side data. Figure 1 displays the mean

\footnotetext{
31 For clarity, throughout this paper we use the letter $\delta$ to denote measurement uncertainties, and $\sigma$ to denote dispersions of a sample of measurements or dispersions (widths) of line profiles.
} 


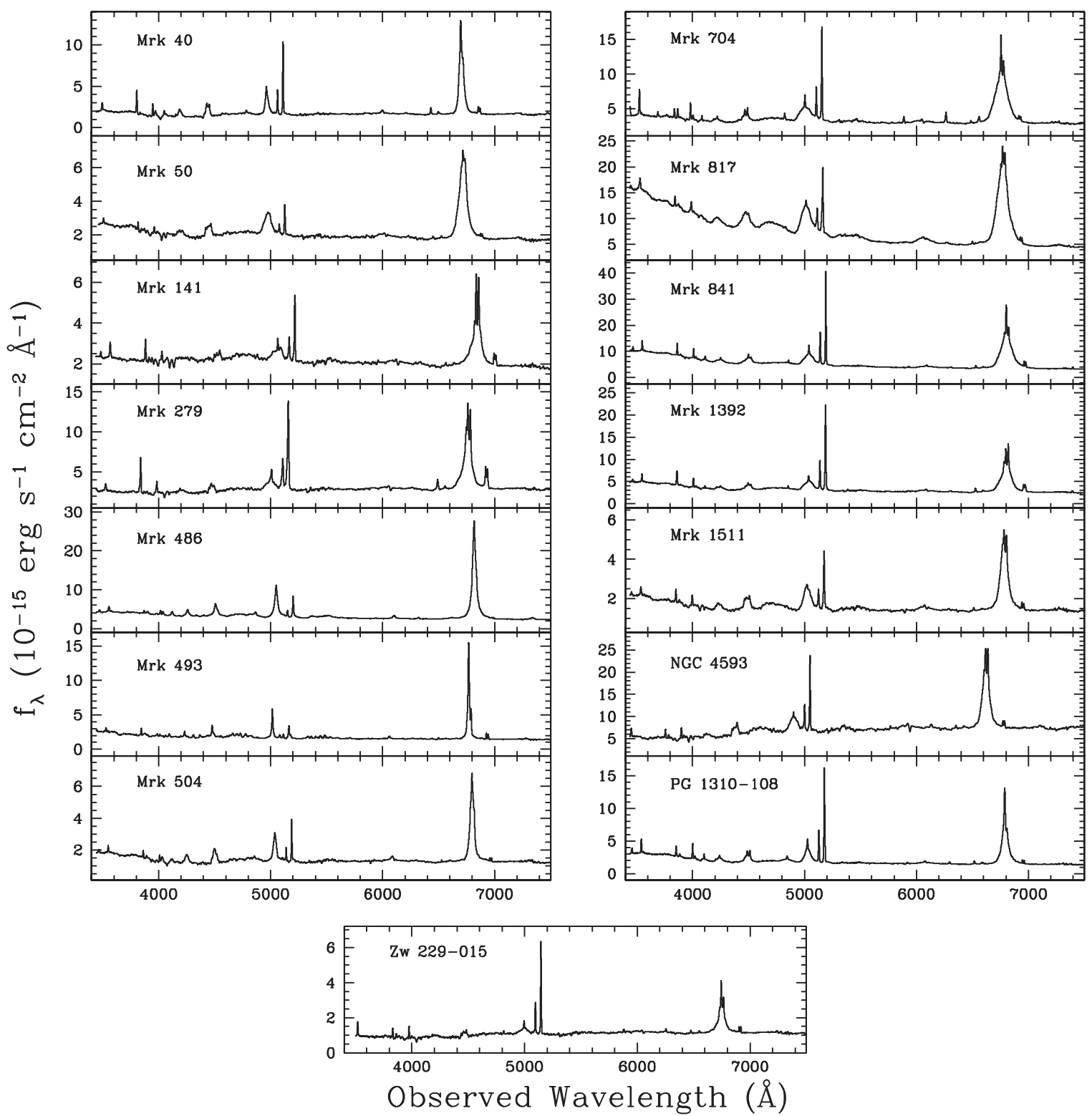

Figure 1. Mean spectra of the sample including data from the blue-side and red-side cameras. The displayed wavelength range is truncated at $7500 \AA$.

spectra of each AGN from the blue and red camera data combined.

For a few of the AGNs, we found that $\mathrm{H} \alpha$ light curves measured from these rescaled spectra had noticeably larger scatter in flux values than the $\mathrm{H} \beta$ light curves. This large excess scatter for the red-side data probably arises from a combination of the nonparallactic slit orientation, the seeing-dependent ratio of AGN to host-galaxy continuum flux, and the fact that the spectral normalization is optimized for wavelengths close to [O III]. Large excess scatter was particularly noticeable in the $\mathrm{H} \alpha$ light curves of Mrk 40 and Mrk 279. For both of these objects, we applied the van Groningen \& Wanders (1992) scaling method separately to the red-side spectra, using the $[\mathrm{O} \mathrm{I}]$ $\lambda 6300$ line as a reference. This significantly improved the quality of the $\mathrm{H} \alpha$ light curves for these two objects, although a few extreme outlier points were still present in the data. For other objects, we found that either rescaling using $\left[\mathrm{O}_{\mathrm{I}}\right]$ did not noticeably improve the $\mathrm{H} \alpha$ light curves, or, in some cases such as Mrk 50, $[\mathrm{O} \mathrm{I}]$ was too weak to use as a reference line in the spectral scaling. We experimented with using the $\left[\mathrm{S}_{\mathrm{II}}\right]$ doublet as the reference line, but this produced poor results because [S II] is very weak in most of these AGNs and is usually blended with the red wing of $\mathrm{H} \alpha$.

\subsection{Spectral Fitting}

Traditionally, measurements of emission-line fluxes for reverberation mapping have been carried out by selecting two regions nearly free of emission lines on either side of the emission line of interest, then fitting and subtracting a straight line to model and remove the continuum underlying the emission line. While this procedure is simple to implement and can generally produce accurate light curves for strong emission lines, there are several drawbacks associated with it. A straightline fit does not adequately represent the shape of the starlight continuum, which often makes a significant contribution to the spectra of Seyfert 1 nuclei (particularly when observed through a wide slit). Stellar absorption features in the host-galaxy 
starlight produce wiggles and bumps that become superposed on the broad $\mathrm{H} \beta$ line profile (including stellar $\mathrm{H} \beta$ absorption), and these contaminating features are not removed when a straight-line continuum is assumed. Furthermore, while there are some regions around $\mathrm{H} \beta$ where the flux from other emission lines is relatively low, there may still be contributions from weak features such as $\mathrm{Fe}$ II or He II in the selected continuum fitting regions, and it is effectively impossible to define a true continuum region that is entirely devoid of AGN emission features. These issues can compromise measurements of emission-line light curves and broad-line widths or profile shapes in the mean and rms spectra. Measurements of velocityresolved lags in the $\mathrm{H} \beta$ line can be compromised by blending with $\mathrm{He}$ II, $\mathrm{He}$ I, and $\mathrm{Fe}_{\mathrm{II}}$ if these features are not removed before measurement of the reverberation signals, and biased determination of the true continuum level owing to contamination by blended lines or starlight could affect measurements of the lag in the faint high-velocity wings of $\mathrm{H} \beta$. In addition to strong lines such as $\mathrm{H} \beta$, it is also of interest to measure light curves for weaker features including Fe II or He II, but these lines can be severely blended with each other as well as with starlight and other spectral components, and a straight-line continuum fit is usually not adequate for isolating the contributions of weaker broad lines to the spectrum.

In order to address these problems, we have implemented a spectral fitting method which, when applied to each nightly spectrum from a reverberation campaign, decomposes the data into independent spectral components. Fitting of AGN spectra to deblend line and continuum components (including the broad $\mathrm{Fe}$ II blends) has a long history with many different implementations in the literature (e.g., Wills et al. 1985; Dietrich et al. 2002; Woo et al. 2006; Greene \& Ho 2007; Shen et al. 2008; Kovačević et al. 2010), but only recently have such methods been applied to time-series spectroscopic data from reverberation campaigns. The procedure described below is an updated version of the method applied to our data in earlier papers in this series (Barth et al. 2011b, 2013) with several minor modifications and improvements. The method described by Park et al. (2012a) uses a different fitting procedure and a different set of model components, but we find that measurements of $\mathrm{H} \beta$ light curves and other parameters are very similar when carried out using the results of the two independent fitting codes when applied to the same set of input spectra.

The primary goal of our procedure is to provide an accurate fit to the region surrounding $\mathrm{H} \beta$ so that the broad $\mathrm{H} \beta$ profile can be isolated from other features. Consequently, accurate fitting over a small wavelength region is a higher priority than fitting the entire available wavelength range of the observations. Fits to a larger wavelength region (e.g., Denney et al. 2009a) would include several additional emission lines that are of little direct interest for the reverberation measurement, and extending the fitting region blueward could compromise the accuracy of the fit in the $\mathrm{H} \beta$ region owing to the complexity of the spectrum at bluer wavelengths. We fit only the blue-side spectra, and the redward limit of the fits is set by the red end of the blue-side CCD data. Fits were carried out over a rest wavelength range of approximately 4200-5300 $\AA$, with slight differences for each AGN depending on redshift, and the fitting procedure is applied to the data after scaling by the van Groningen \& Wanders (1992) procedure. The Levenberg-Marquardt routines in the IDL mpfit package (Markwardt 2009) are employed for $\chi^{2}$ minimization to optimize the fits, using the propagated error spectra for determination of $\chi^{2}$. The model components and parameters are as follows.

Starlight: we used an $11 \mathrm{Gyr}$, solar metallicity, single-burst spectrum from Bruzual \& Charlot (2003) to model the hostgalaxy starlight. We additionally carried out tests in which a younger (290 Myr) population component was added. Its contribution was generally small and not well constrained, and it was omitted in the final fits. During the fitting process, the starlight spectrum was rebinned to a log-linear wavelenth scale, broadened by convolution with a Gaussian kernel, and then rebinned again to match the linear wavelength scale of the data. Free parameters include the flux normalization of the starlight spectrum, the redshift, and the velocity width of the broadening kernel. Since the spectral resolution of the Bruzual \& Charlot (2003) spectra differs from the Lick data, the convolution kernel width does not directly correspond to the actual stellar velocity dispersion in the host galaxy. The velocity-broadening dispersion parameter was limited to a maximum value of $350 \mathrm{~km} \mathrm{~s}^{-1}$ in order to prevent it from increasing to unphysically large values; this limit was reached only for Mrk 486, in which stellar absorption features are essentially invisible over the wavelength range of the fits.

AGN featureless continuum: the AGN continuum was modeled as a power law, with free parameters including the flux normalization at rest wavelength $5100 \AA$ and the powerlaw spectral index. Over the wavelength range that was fitted, Balmer continuum emission does not contribute to the spectrum. We do not ascribe any physical meaning to nightto-night changes in the best-fitting spectral index, since these can be caused by wavelength-dependent slit losses resulting from differential atmospheric refraction and variable seeing.

$\left[O_{\text {III }}\right]$ : a fourth-order Gauss-Hermite function (van der Marel \& Franx 1993) was used to model the $\left[\mathrm{O}_{\text {III }}\right] \lambda 5007$ profile. Free parameters included the amplitude, centroid wavelength, line dispersion, and the higher-order moments $h_{3}$ and $h_{4}$. The $4959 \AA$ line was modeled with the same velocity profile and an amplitude fixed to $1 / 3$ of that the $5007 \AA$ line, and a fixed rest-frame wavelength separation of $47.9 \AA$. A fourth-order Gauss-Hermite function proved to be adequate for modeling the $[\mathrm{O}$ III] profiles well in all but one of the AGNs; as described below, we modified the set of fit components for Mrk 279.

$\mathrm{H} \beta$ : for the broad component of $\mathrm{H} \beta$, a fourth-order GaussHermite function was used. This provides sufficient flexibility to fit the broad-line profiles for nearly all of the AGNs well, with the exception of objects such as Mrk 141 having distinct shoulders or humps on their profiles. Free parameters include the broad component centroid, dispersion, $h_{3}, h_{4}$, and amplitude. The narrow component was set to have a profile identical to $[\mathrm{O} \mathrm{III}]$, but a small shift was allowed in the wavelength centroid of the narrow component as another free parameter.

$H e$ II: the He II $\lambda 4686$ line was modeled as the sum of two Gaussians representing the broad and narrow components. For each component, free parameters included the centroid, dispersion, and amplitude. Initial trial fits indicated that the broad and narrow components sometimes had offset centroid wavelengths, and for some AGNs the fits failed noticeably when the two components were forced to have the same centroid. In some cases this might be the result of broad He I $\lambda 4713$ being present in the data but not included as a fit 
component. If the broad $\mathrm{He}$ II model component incorporated some He I $\lambda 4713$ flux then this would induce a spurious but small redward shift relative to its true wavelength. Véron et al. (2002) illustrate examples of fits that include both He II $\lambda 4686$ and $\mathrm{He}_{\mathrm{I}} \lambda 4713$, but since these features are badly degenerate in broad-lined AGNs we do not attempt to include the He I $\lambda 4713$ line in our fits as a separate component.

$\mathrm{He}$ I: we included the possible contributions of $\mathrm{He}_{\mathrm{I}}$ broad lines at 4471, 4922, and $5016 \AA$ rest wavelength. In order to keep the number of free parameters to a minimum for these relatively weak lines, we used Gaussian profiles and followed Vestergaard \& Peterson (2005) in setting the 4922 and $5016 \AA$ lines to have the same amplitude, but we did not force any relationship between the amplitudes of these lines and the $4471 \AA$ line. All three He I lines were assumed to have identical velocity widths, and their velocity centroids were set to be identical to that of $\mathrm{H} \beta$. The He I components thus required only three additional free parameters to represent the amplitudes and widths of these features. The He I 4922 and $5016 \AA$ lines are in the "red shelf" of $\mathrm{H} \beta$, and are blended with the red wing of $\mathrm{H} \beta$, the [O III] lines, and with Fe II features (Véron et al. 2002). In fact the 4922 and $5016 \AA$ lines are nearly degenerate with two Fe II features that appear in the iron templates. The amplitude of these two features in the fits depends strongly on which Fe II template is chosen. While the sum of the Fe II and He I fluxes in this spectral region is fairly well determined by the fits, we do not attempt to extract $\mathrm{He}$ I light curves because of the ambiguity in separating these features from the Fe II emission. For most AGNs, the best-fitting amplitude of the $\mathrm{He}_{\mathrm{I}} \lambda 4471$ line is either zero or negligibly small, regardless of Fe II template choice.

$\mathrm{Fe}$ II: the blends of broad Fe II emission were modeled using template spectra, including those described by Boroson \& Green (1992), Véron-Cetty et al. (2004), and Kovačević et al. (2010). We carried out fits separately with each of these templates in order to examine systematic differences in the results. Template spectra were convolved with a Gaussian kernel in velocity space, similar to the broadening of the starlight spectrum described above. In each case, free parameters included the Fe II velocity shift relative to broad $\mathrm{H} \beta$, the broadening kernel width, and the normalization of the broadened template spectrum. The Boroson \& Green (1992) and Véron-Cetty et al. (2004) templates are monolithic and only one normalization parameter is required. The Kovačević et al. (2010) template (see also Shapovalova et al. 2012) is composed of five separate templates representing different emission multiplet groups, and a normalization parameter is required for each one. In a given spectral fit using the Kovačević et al. (2010) templates, each of the five templates was assumed to have the same velocity broadening and velocity shift. The Kovačević et al. (2010) templates are distributed with a discrete sequence of velocity broadenings, and we used the narrowest templates (corresponding to $700 \mathrm{~km} \mathrm{~s}^{-1}$ broadening) and then applied a Gaussian velocity broadening during the fitting process as described above. Examination of the fits for a very narrow-lined AGN such as Mrk 493 clearly illustrate the contribution of Fe II in the red wings of both $\mathrm{H} \beta$ and $[\mathrm{O} \mathrm{III}] \lambda 5007$, while in more typical broad-lined AGNs these Fe II features are more difficult to identify individually and their contribution to this spectral region may be less obvious unless a multicomponent fit is carried out.
Reddening: all model components were reddened by a Cardelli et al. (1989) reddening law, with $E(B-V)$ as a free parameter in the fit. This parameter accounts for possible reddening within the AGN host galaxy as well as Galactic reddening, and also accounts for possible errors in relative flux calibration resulting from the nonparallactic slit orientation or other wavelength-dependent flux calibration errors. For some AGNs, the best-fitting values of $E(B-V)$ varied significantly from night to night, and we do not interpret the fitted parameter values as actual measurements of the reddening toward the AGNs. In principle, the AGN continuum and BLR, NLR, and host-galaxy light could have different line of sight reddening, but the data would not provide sufficient constraints to obtain meaningful values of reddening for these components independently.

In the $\mathrm{H} \beta$ and $[\mathrm{O} \mathrm{III}]$ models, the wings of Gauss-Hermite functions can extend to negative values for certain values of $h_{3}$ and $h_{4}$. Negative values were clipped and replaced with zero. This only affects the distant wings of line profiles and has a very minor effect on the fits.

Other emission lines are present in the spectrum that could be fitted by adding components and free parameters to the model, but we chose to restrict the number of components to those listed above in order to avoid either slowing down the fitting procedure or introducing additional fitting degeneracies. Instead, other emission features were accounted for simply by setting a wavelength region around those features to have zero weight in the fits. The largest feature that was masked out was the blend of $\mathrm{H} \gamma$ and $[\mathrm{O}$ III $] \lambda 4363$. This blend typically has a complex shape that, if included in the fit, would require three overlapping Gauss-Hermite or Gaussian components and potentially nine or more additional free parameters. The masked region corresponded to $4280-4400 \AA$ (rest wavelength) but was adjusted in a few cases for objects having very broad lines. We also masked out a small region around the narrow $\left[\mathrm{N}_{\mathrm{I}}\right]$ lines at 5199 and $5201 \AA$.

Our procedure fits all of these spectral components to the data simultaneously, in contrast to other methods which fit and remove continuum and $\mathrm{Fe}$ II components prior to fitting the $\mathrm{H} \beta$ profile (e.g., Shen et al. 2008; Park et al. 2012a). Fits were carried out separately using each of the three Fe II templates. The total number of free parameters in the fits is 29 when the monolithic Fe II templates are used, or 33 when the multicomponent Kovačević et al. (2010) templates are used. For each AGN, the mean spectrum was fitted first, and then the best-fit parameters determined for the high $\mathrm{S} / \mathrm{N}$ mean spectrum were used as the initial parameters for the fits to each nightly spectrum. In most cases, fits using the Kovačević et al. (2010) templates returned the best $\chi_{\nu}^{2}$ values, and we use these templates for the final fit results presented in this paper. Figure 2 illustrates the systematic differences between the three templates when fitted to the spectrum of Mrk 493, which has the strongest and narrowest Fe II lines of the AGNs in our sample. The template differences are most pronounced at wavelengths underlying the broad $\mathrm{H} \gamma$ and $\mathrm{H} \beta$ lines and the $\mathrm{H} \beta$ red shelf region.

The fitting procedure was modified for Mrk 279. Its [O III] lines are unusually broad with strong asymmetric blue wings which contribute substantially to the "red shelf" region redward of $\mathrm{H} \beta$, and the 4th-order Gauss-Hermite model gave a poor fit to the $[\mathrm{O} \mathrm{III}]$ doublet leaving very strong residuals in this region. For this object, we removed the He I components from the 


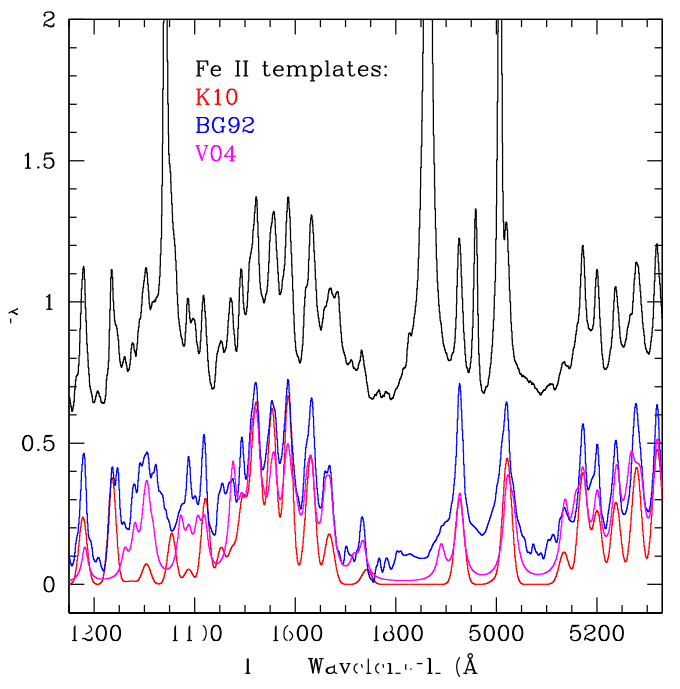

Figure 2. Illustration of the differences between the Fe II templates of Boroson \& Green (1992; blue), Véron-Cetty et al. (2004; magenta), and Kovačević et al. (2010; red) when fitted to the spectrum of Mrk 493. The Mrk 493 mean spectrum, after subtraction of the AGN featureless continuum and starlight components, is shown in black, and has been shifted upward by 0.7 units for clarity. The colored curves show the Fe II templates with velocity broadening and normalization as determined from the best fit to the mean spectrum.

model (since they did not appear to be necessary in this case) and replaced them with two additional Gaussian components representing the blue wings of the $[\mathrm{O}$ III $] \lambda \lambda 4959,5007$ lines. The $\mathrm{H} \beta$ model also required modifications. In order to prevent the broad $\mathrm{H} \beta$ width from blowing up to arbitrarily high values due to fitting degeneracy in the red wing, we restricted the broad component model to $\sigma<30 \AA$. We also found that the narrow component of $\mathrm{H} \beta$ had a significantly different profile from $[\mathrm{O}$ III] in Mrk 279, and allowed the width of narrow $\mathrm{H} \beta$ to vary rather than tying it to the [O III] width. This resulted in $\sigma$ $\left(\mathrm{H} \beta_{n}\right) \approx 0.75 \sigma([\mathrm{O} \mathrm{III}])$.

The best fits to the mean spectrum of each AGN, using the Kovačević et al. 2010 template, are shown in Figure 3.

For each spectrum, the best-fitting model components are saved. Additionally, we save the decomposed $\mathrm{H} \beta$ profile, made by subtracting all model components other than $\mathrm{H} \beta$ from the original spectrum. This spectrum also contains the $\mathrm{H} \gamma+[\mathrm{O}$ III $]$ blend as part of the fitting residual. We save two versions of this spectrum, one including and one excluding the narrow $\mathrm{H} \beta$ component. These decomposed spectra (rather than the noise-free models) are used for measurement of the $\mathrm{H} \beta$ light curves and line widths. Figure 4 illustrates examples of the decomposed $\mathrm{H} \beta$ profiles and component models for six AGNs from a single night's data.

The fitting procedure returns estimates of uncertainties on each of the free parameters. However, for measurements of emission-line light curves, what is needed is an estimate of the uncertainty on the flux density at each pixel for a particular spectral component such as $\mathrm{H} \beta$. We employed a Monte Carlo procedure to estimate the flux uncertainties. For each spectrum, after finding the best model fit, we created 100 modified versions of the spectrum by adjusting each pixel by a random Gaussian deviate drawn from a distribution with a Gaussian dispersion equal to the amplitude of the propagated error spectrum at that pixel. The spectral fit was then repeated for each of the 100 randomly deviated spectra, and the individual fit components were saved. For each spectral component, the value of the error spectrum at a given pixel was taken to be the standard deviation of the flux values from the 100 re-fitted versions of that spectral component. Similarly, we produced error spectra for the decomposed $\mathrm{H} \beta$ profiles by taking the standard deviation of the 100 Monte Carlo realizations of the decomposed profile (i.e., the deviated spectrum minus all best-fit components other than $\mathrm{H} \beta$ ).

From this procedure, we found that the error spectrum of the decomposed $\mathrm{H} \beta$ profile has an amplitude at each spectral pixel that is nearly equal to that of the original error spectrum, but noisier as a result of the limited number of Monte Carlo iterations. Based on the results of this procedure, we adopt the original error spectrum as our best estimate of the pixel uncertainties on the individual emission-line component spectra that we use to measure light curves. The error spectra do not incorporate possible systematic uncertainties due to differences between $\mathrm{Fe}$ II templates or the choice of a particular host-galaxy starlight model.

Using the fit results, we constructed revised mean and rms spectra. Figure 5 shows the original mean and rms spectra along with mean and rms spectra constructed after subtraction of the best-fitting starlight and AGN featureless continuum components from each nightly spectrum. In some cases such as Mrk 279, the $\mathrm{H} \beta$ profile in the rms spectrum changes significantly after subtraction of the continuum components; similar results have previously been discussed by Park et al. (2012a). Additionally, for some AGNs the He II profiles in rms spectra are significantly altered by removal of the continuum components; these changes are particularly prominent in Mrk 504 and Mrk 1511. Another version of the rms spectrum was constructed by subtracting all model components other than broad $\mathrm{H} \beta$ from the nightly spectra. This version isolates the variability of the broad $\mathrm{H} \beta$ line itself, and was used to measure the width of the $\mathrm{H} \beta \mathrm{rms}$ profile as described in Section 6 . Figure 6 shows multiple versions of the rms spectrum for Mrk 40 to illustrate the changes when different spectral components are subtracted prior to calculating the rms flux. One advantage of subtracting narrow emission-line components prior to constructing the rms spectrum is that the residual noise from these intrinsically nonvariable features is significantly reduced. This is clearly apparent for the [O III] lines in Mrk 40 (Figure 6).

In future work, a variety of possible extensions and improvements to the fitting method can be explored. These include (a) addition of model components for other emission lines such as $\mathrm{H} \gamma$ and [O III] $\lambda 4363$ instead of simply masking them out from the fit; (b) broadening the wavelength range of the fit blueward down to the near-UV atmospheric cutoff, and redward to $\mathrm{H} \alpha$; (c) inclusion of multiple-age stellar populations; (d) addition of a Balmer continuum component for fits extending below the Balmer break; (e) use of higherorder models to achieve more accurate fits to strong lines such as $[\mathrm{O}$ III $] \lambda 5007$ and for irregular or double-peaked broad Balmer lines; (f) exploration of different methods for constraining the decomposition in the $\mathrm{H} \beta$ red shelf region; (g) allowing for the possibility that different reddening values or even different reddening laws might apply to spectral components originating at different spatial scales. The possible benefits of these improvements must however be weighed against the increased complexity that comes with adding more free parameters and the degeneracies among 

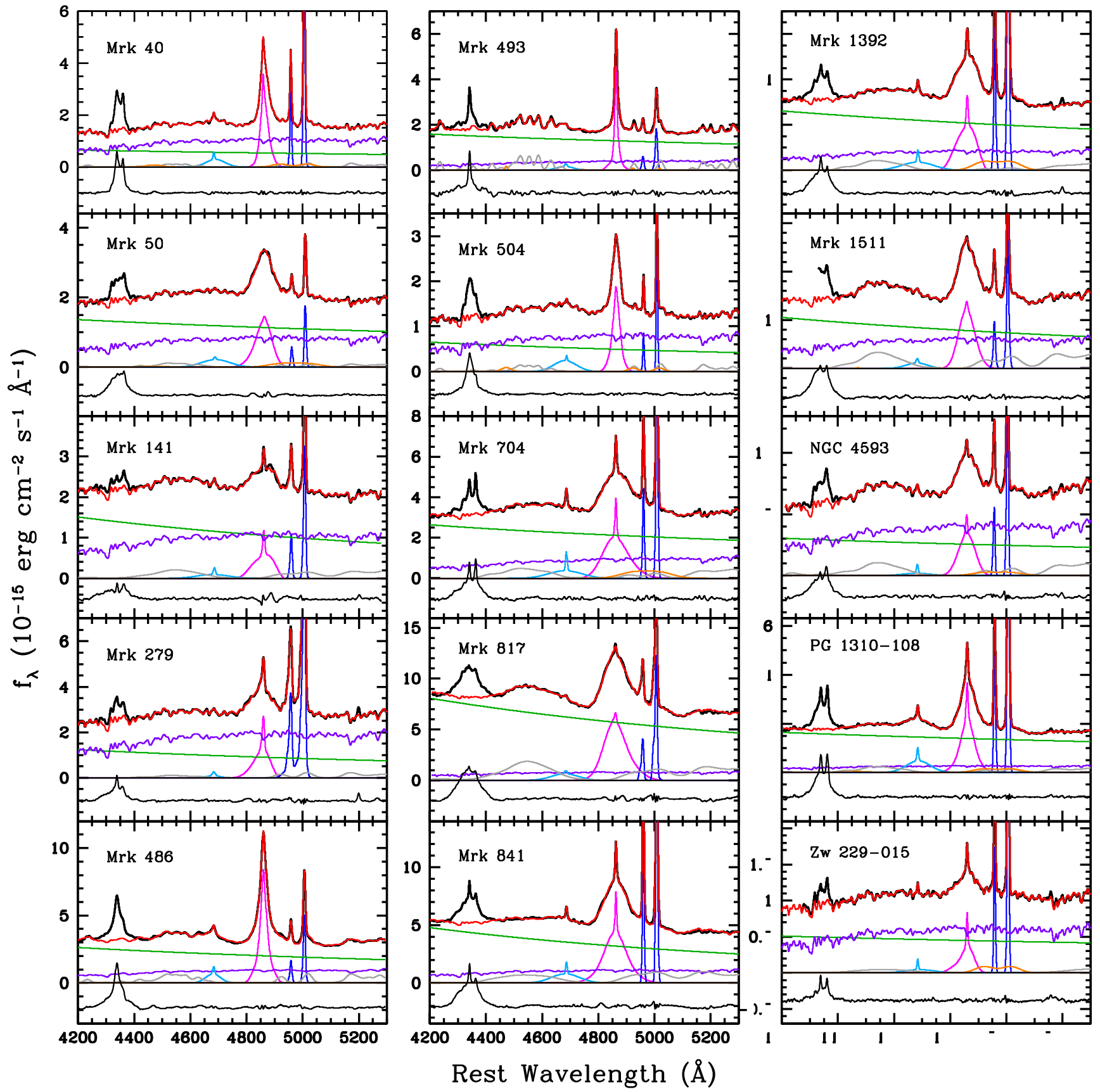

Figure 3. Fits to the blue-side mean spectra. The observed mean spectra are shown in black. Model components include starlight (purple), the AGN featureless continuum (green), $\mathrm{H} \beta$ (magenta), He II (cyan), He I (orange), Kovačević et al. (2010) Fe II template (gray), and [O III] (blue). The sum of all model components is shown in red, superposed on the observed spectrum. The residual spectrum (data-total model) is shown in black and is offset downward to negative values for clarity. This residual contains emission features including $\mathrm{H} \gamma,[\mathrm{O} \mathrm{II}] \lambda 4363$, and $[\mathrm{N} \mathrm{I}] \lambda 5200$ that are not incorporated as components in the model.

them, particularly for the continuum components. Furthermore, since the primary goal is often to achieve the most accurate fit to the wavelength region surrounding $\mathrm{H} \beta$, care must be taken to ensure that fitting a broader wavelength range does not worsen the fit in this region. The available set of $\mathrm{Fe}$ II templates remains a fundamental limitation and source of systematic uncertainty in the fits, and further work to develop more flexible sets of $\mathrm{Fe}$ II templates, both theoretical and emipirical, covering the full UV/optical wavelength range, would be extremely valuable for future reverberation mapping.

\section{EMISSION-LINE AND CONTINUUM LIGHT CURVES}

Light curves for broad emission lines were measured by direct integration of spectra. The epoch of each observation is given by the Heliocentric Julian Date (HJD) determined from the UT date saved in the FITS image headers, using the IRAF setjd routine. To measure the $\mathrm{H} \beta$ and $\mathrm{H} \gamma$ light curves for each night's observation of an AGN, we began with the observed spectrum and subtracted all of the best-fitting model components except for the broad and narrow $\mathrm{H} \beta$ models. This left a residual spectrum with a continuum level of zero (modulated by the fitting residuals) and the $\mathrm{H} \beta$ line and $\mathrm{H} \gamma$ 


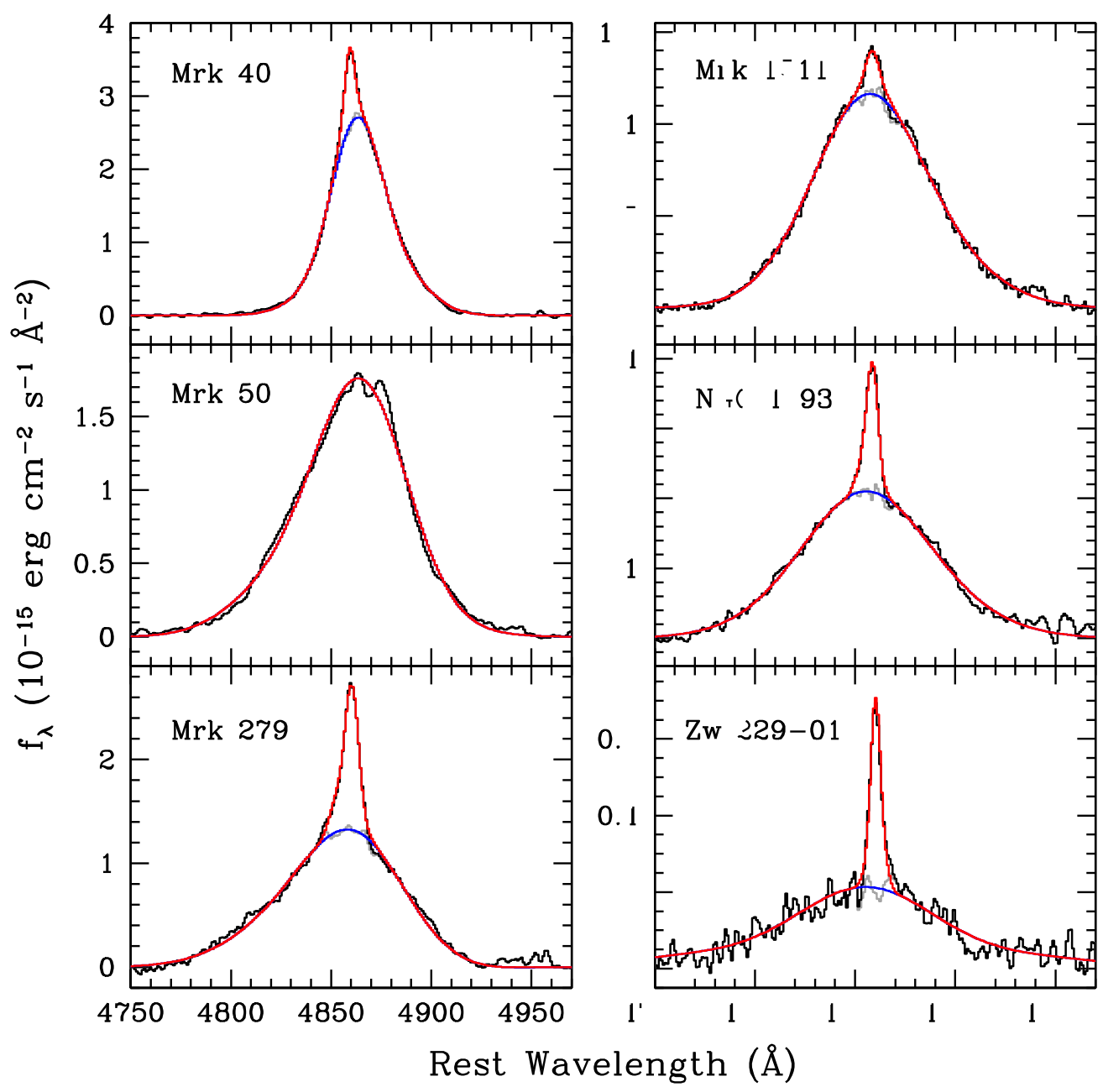

Figure 4. Examples of fits to the $\mathrm{H} \beta$ line in single-epoch observations. The displayed data are from observations taken on 2011 April 27. In each panel, the decomposed $\mathrm{H} \beta$ profile obtained by subtracting all model components other than $\mathrm{H} \beta$ from the spectrum is shown in black. This includes both the narrow and broad components of $\mathrm{H} \beta$. The broad component spectrum after additionally subtracting the narrow $\mathrm{H} \beta$ model component is shown in gray. Blue and red curves show the best-fitting broad $\mathrm{H} \beta$ and total $\mathrm{H} \beta$ (broad + narrow) model components.

$+[\mathrm{O}$ III $]$ blends as the remaining emission features. The flux in these features was integrated over wavelength ranges that were selected for each AGN to encompass the full width of the line profiles. Wavelength ranges for each light curve are listed in Table 3. We measure the total (broad + narrow) flux for $\mathrm{H} \beta$ in order to avoid introducing possible errors into the light curves due to degeneracy in decomposing the two components, consistent with methods typically used for reverberation measurements (e.g., Kaspi et al. 2000; Bentz et al. 2009b). The narrow component adds a constant flux pedestal to the light curves. The $\mathrm{H} \gamma$ measurement includes the flux of the broad and narrow $\mathrm{H} \gamma$ lines as well as $\left[\mathrm{O}_{\mathrm{III}}\right] \lambda 4363$.

We also measured light curves for He II $\lambda 4686$ and for the Fe II blends integrated over the rest wavelength range $4400-4700 \AA$. The light curves for these weak features were measured from the best-fitting model component spectra for each night's data, and for $\mathrm{He}$ II the light curves include both the broad and narrow components. The $\mathrm{Fe}$ II emission was integrated over the rest wavelength ranges of 4400-4700 and $5100-5400 \AA$, avoiding regions in which $\mathrm{Fe}_{\text {II }}$ emission is degenerate with $\mathrm{H} \beta$ or the possible $\mathrm{He}$ I emission in the $\mathrm{H} \beta$ red shelf.

For the $\mathrm{H} \alpha$ and $\mathrm{H} \delta$ lines, which lie outside the wavelength range of the spectral fits, we measured fluxes from the scaled spectra using the traditional approach of fitting a local linear continuum to surrounding line-free regions on either side of the line. The $\mathrm{H} \alpha$ light curves include the fluxes of the narrow [N II] $\lambda 6548,6583$ lines.

Light curves are displayed in Figures 7-14, and the light curve data are listed in Table 4.

Light curves were also measured for the AGN continuum in the $U$-band spectral region, since the AGN featureless continuum tends to dominate over starlight in this region and is more highly variable than at redder wavelengths. The mean continuum flux density was measured over $\lambda_{\text {rest }}=3500$ $3600 \AA$, and we refer to this spectroscopic continuum flux as the $U_{s}$ band. For a few objects, this wavelength range was adjusted in order to avoid emission lines. This measurement includes both AGN and starlight continuum. Table 3 lists the wavelength range that was integrated for each light curve. Since this wavelength range is far from the wavelength of [O III], which was used as the flux calibration reference for spectral scaling, the relative accuracy of the photometric calibration is sometimes poor. From the appearance of the $U_{s}$ light curves, the flux uncertainties in portions of the light curves are $\sim 10 \%$ or occasionally worse. The scatter in the $U_{s}$ light curves for some objects (such as Mrk 50) is highest at 

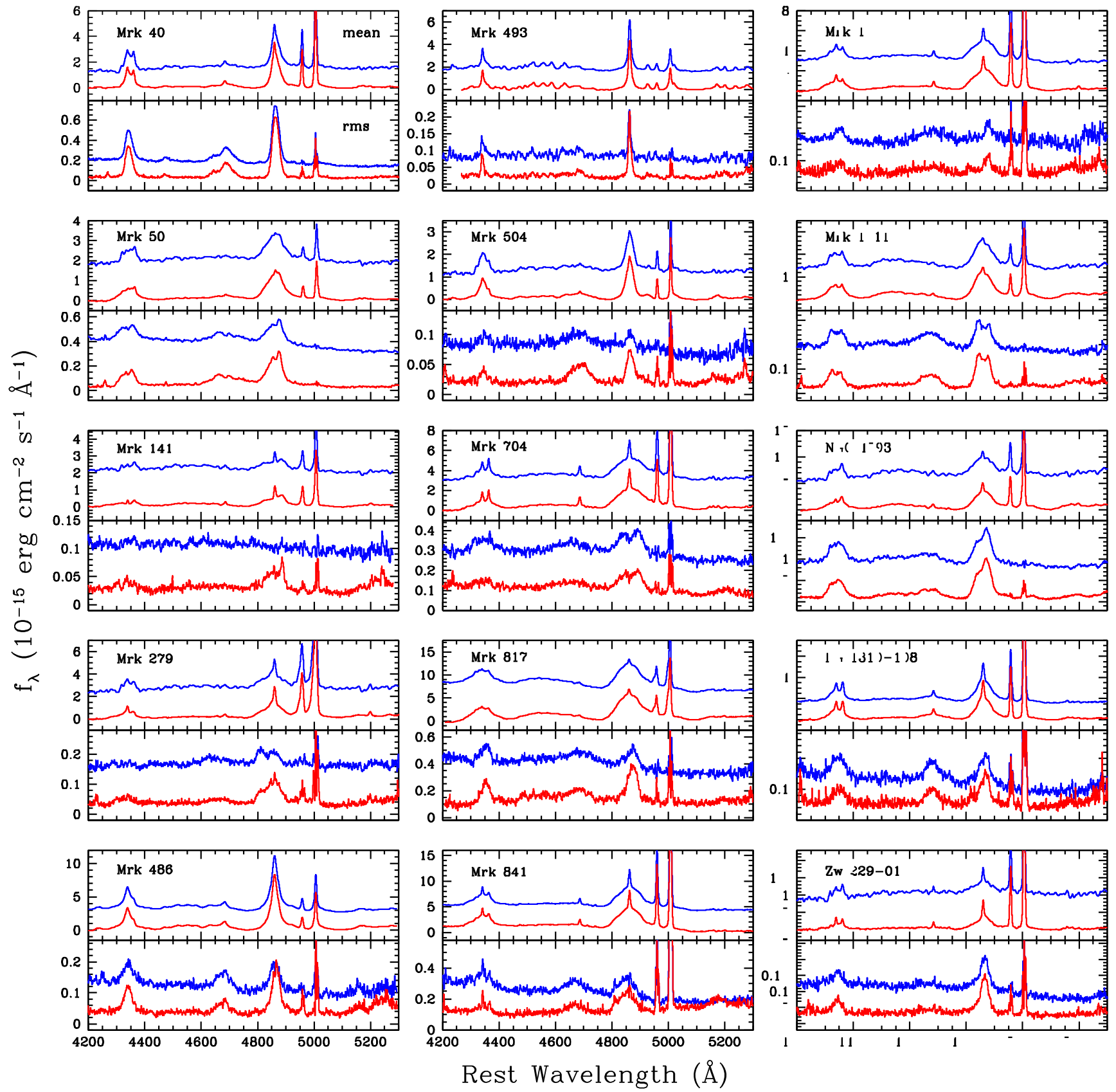

Figure 5. Mean and rms spectra for the blue-side fitting region. In each panel, the upper subpanel shows shows the standard mean spectrum (blue), and a "line-only" mean spectrum constructed after subtraction of the AGN featureless continuum and starlight components from each nightly spectrum (red). The lower subpanel shows the standard rms spectrum (blue), and an rms spectrum constructed after subtraction of the AGN featureless continuum and starlight components from each nightly spectrum (red).

times close to the start and end of the campaign, when targets were observed at higher airmasses, but this trend does not appear to hold for the entire sample.

From each light curve, we calculated the statistics $F_{\mathrm{var}}$ and $R_{\max }$ to characterize the variability amplitude, and for comparison with previous reverberation campaigns (e.g., Bentz et al. 2009b). The quantity $F_{\mathrm{var}}$ is similar to the normalized excess scatter and is defined as

$$
F_{\mathrm{var}}=\frac{\sqrt{\sigma_{f}^{2}-\delta_{\mathrm{rms}}^{2}}}{\langle f\rangle},
$$

where $\sigma_{f}^{2}$ is the variance of the flux values in the light curve, $\delta_{\mathrm{rms}}$ is the rms measurement uncertainty on the fluxes, and $\langle f\rangle$ is the mean flux (Rodríguez-Pascual et al. 1997; Edelson et al. 2002). $R_{\max }$ gives the maximum variability amplitude and is simply the ratio of maximum to minimum fluxes in the light curve. To account for the contribution of residual scatter from the spectral scaling to the error budget, we modified the flux uncertainties by taking the product of excess [O $\mathrm{III}]$ flux scatter with mean flux $\left(\sigma_{\mathrm{nx}} \times\langle f\rangle\right)$ and adding that quantity in quadrature to each individual flux uncertainty value prior to calculating $F_{\mathrm{var}}, R_{\max }$, and their uncertainties. As an overall measure of light-curve variability, $F_{\mathrm{var}}$ is preferable to $R_{\max }$ 
Table 3

Light Curve Wavelength Ranges and Variability Statistics

\begin{tabular}{|c|c|c|c|c|}
\hline Galaxy & $\begin{array}{l}\text { Light } \\
\text { Curve }\end{array}$ & $\begin{array}{l}\text { Rest Wave- } \\
\text { lengths }(\AA)\end{array}$ & $F_{\mathrm{var}}$ & $R_{\max }$ \\
\hline \multirow[t]{7}{*}{ Mrk 40} & $U_{s}$ & $3500-3600$ & $0.30 \pm 0.03$ & $2.78 \pm 0.04$ \\
\hline & $\mathrm{H} \alpha$ & $6493-6660$ & $0.14 \pm 0.02$ & $2.02 \pm 0.02$ \\
\hline & $\mathrm{H} \beta$ & $4799-4916$ & $0.20 \pm 0.02$ & $1.98 \pm 0.03$ \\
\hline & $\mathrm{H} \gamma$ & 4309-4387 & $0.21 \pm 0.02$ & $2.04 \pm 0.04$ \\
\hline & $\mathrm{H} \delta$ & $4074-4142$ & $0.27 \pm 0.03$ & $2.61 \pm 0.06$ \\
\hline & $\mathrm{He}_{\text {II }}$ & $4583-4789$ & $0.38 \pm 0.04$ & $3.57 \pm 0.21$ \\
\hline & $\mathrm{Fe}$ II & $\begin{array}{c}4400-4700 \\
5100-5400\end{array}$ & $0.15 \pm 0.02$ & $2.05 \pm 0.10$ \\
\hline \multirow[t]{7}{*}{ Mrk 50} & $U_{s}$ & $3500-3600$ & $0.34 \pm 0.03$ & $3.88 \pm 0.34$ \\
\hline & $\mathrm{H} \alpha$ & 6449-6693 & $0.10 \pm 0.01$ & $1.53 \pm 0.08$ \\
\hline & $\mathrm{H} \beta$ & $4758-4944$ & $0.20 \pm 0.02$ & $2.17 \pm 0.13$ \\
\hline & $\mathrm{H} \gamma$ & $4270-4417$ & $0.23 \pm 0.02$ & $2.50 \pm 0.17$ \\
\hline & $\mathrm{H} \delta$ & $4065-4133$ & $0.29 \pm 0.03$ & $2.93 \pm 0.23$ \\
\hline & $\mathrm{He}$ II & $4495-4817$ & $0.55 \pm 0.05$ & $12.02 \pm 2.09$ \\
\hline & $\mathrm{Fe}$ II & $\begin{array}{c}4400-4700 \\
5100-5400\end{array}$ & $0.35 \pm 0.03$ & $7.28 \pm 1.31$ \\
\hline \multirow[t]{2}{*}{ Mrk 141} & $U_{s}$ & $3500-3600$ & $0.09 \pm 0.01$ & $1.45 \pm 0.03$ \\
\hline & $\mathrm{H} \beta$ & $4782-4925$ & $0.08 \pm 0.01$ & $1.39 \pm 0.03$ \\
\hline \multirow[t]{6}{*}{ Mrk 279} & $U_{s}$ & $3500-3600$ & $0.13 \pm 0.02$ & $1.63 \pm 0.02$ \\
\hline & $\mathrm{H} \alpha$ & $6405-6667$ & $0.04 \pm 0.01$ & $1.22 \pm 0.01$ \\
\hline & $\mathrm{H} \beta$ & $4755-4920$ & $0.07 \pm 0.01$ & $1.31 \pm 0.01$ \\
\hline & $\mathrm{H} \gamma$ & $4251-4416$ & $0.07 \pm 0.01$ & $1.34 \pm 0.02$ \\
\hline & $\mathrm{H} \delta$ & $4047-4115$ & $0.09 \pm 0.01$ & $1.45 \pm 0.04$ \\
\hline & $\mathrm{He}_{\mathrm{II}}$ & $4590-4765$ & $0.30 \pm 0.04$ & $2.98 \pm 0.21$ \\
\hline \multirow[t]{2}{*}{ Mrk 486} & $U_{s}$ & $3500-3600$ & $0.07 \pm 0.01$ & $1.40 \pm 0.04$ \\
\hline & $\mathrm{H} \beta$ & $4784-4928$ & $0.02 \pm 0.01$ & $1.10 \pm 0.03$ \\
\hline \multirow[t]{2}{*}{ Mrk 493} & $U_{s}$ & $3500-3600$ & $0.04 \pm 0.01$ & $1.20 \pm 0.04$ \\
\hline & $\mathrm{H} \beta$ & 4809-4897 & $0.03 \pm 0.01$ & $1.17 \pm 0.04$ \\
\hline \multirow[t]{2}{*}{ Mrk 504} & $U_{s}$ & $3500-3600$ & $0.07 \pm 0.01$ & $1.36 \pm 0.03$ \\
\hline & $\mathrm{H} \beta$ & 4798-4914 & $0.04 \pm 0.01$ & $1.24 \pm 0.03$ \\
\hline \multirow[t]{6}{*}{ Mrk 704} & $U_{s}$ & $3500-3570$ & $0.13 \pm 0.02$ & $2.02 \pm 0.03$ \\
\hline & $\mathrm{H} \alpha$ & $6422-6704$ & $0.07 \pm 0.01$ & $1.37 \pm 0.01$ \\
\hline & $\mathrm{H} \beta$ & $4770-4936$ & $0.05 \pm 0.01$ & $1.23 \pm 0.01$ \\
\hline & $\mathrm{H} \gamma$ & $4256-4411$ & $0.08 \pm 0.01$ & $1.46 \pm 0.02$ \\
\hline & $\mathrm{H} \delta$ & $4052-4149$ & $0.10 \pm 0.01$ & $1.63 \pm 0.08$ \\
\hline & $\mathrm{He}$ II & $4489-4848$ & $0.28 \pm 0.04$ & $3.62 \pm 0.13$ \\
\hline \multirow[t]{2}{*}{ Mrk 817} & $U_{s}$ & $3500-3600$ & $0.08 \pm 0.01$ & $1.40 \pm 0.03$ \\
\hline & $\mathrm{H} \beta$ & $4750-4944$ & $0.04 \pm 0.01$ & $1.16 \pm 0.02$ \\
\hline \multirow[t]{2}{*}{ Mrk 841} & $U_{s}$ & $3500-3570$ & $0.14 \pm 0.02$ & $1.92 \pm 0.03$ \\
\hline & $\mathrm{H} \beta$ & $4757-4940$ & $0.05 \pm 0.01$ & $1.27 \pm 0.02$ \\
\hline \multirow[t]{2}{*}{ Mrk 1392} & $U_{s}$ & $3500-3600$ & $0.14 \pm 0.02$ & $1.88 \pm 0.03$ \\
\hline & $\mathrm{H} \beta$ & $4758-4932$ & $0.03 \pm 0.01$ & $1.16 \pm 0.01$ \\
\hline \multirow[t]{7}{*}{ Mrk 1511} & $U_{s}$ & $3500-3600$ & $0.19 \pm 0.02$ & $2.42 \pm 0.06$ \\
\hline & $\mathrm{H} \alpha$ & $6432-6693$ & $0.09 \pm 0.01$ & $1.45 \pm 0.03$ \\
\hline & $\mathrm{H} \beta$ & $4768-4933$ & $0.12 \pm 0.01$ & $1.43 \pm 0.03$ \\
\hline & $\mathrm{H} \gamma$ & $4236-4430$ & $0.13 \pm 0.01$ & $1.54 \pm 0.04$ \\
\hline & $\mathrm{H} \delta$ & $4062-4140$ & $0.20 \pm 0.02$ & $2.00 \pm 0.07$ \\
\hline & $\mathrm{He}$ II & $4556-4797$ & $0.54 \pm 0.06$ & $10.80 \pm 1.59$ \\
\hline & $\mathrm{Fe}$ II & $\begin{array}{c}4400-4700, \\
5100-5400\end{array}$ & $0.10 \pm 0.01$ & $1.43 \pm 0.03$ \\
\hline \multirow[t]{7}{*}{ NGC 4593} & $U_{s}$ & $3500-3570$ & $0.38 \pm 0.04$ & $4.54 \pm 0.25$ \\
\hline & $\mathrm{H} \alpha$ & $6442-6690$ & $0.16 \pm 0.02$ & $1.81 \pm 0.07$ \\
\hline & $\mathrm{H} \beta$ & 4757-4926 & $0.23 \pm 0.02$ & $2.11 \pm 0.08$ \\
\hline & $\mathrm{H} \gamma$ & $4242-4410$ & $0.25 \pm 0.03$ & $2.15 \pm 0.09$ \\
\hline & $\mathrm{H} \delta$ & $4058-4133$ & $0.43 \pm 0.05$ & $4.72 \pm 0.50$ \\
\hline & $\mathrm{He}$ II & 4539-4807 & $0.72 \pm 0.08$ & $19.28 \pm 4.41$ \\
\hline & $\mathrm{Fe}$ II & $\begin{array}{c}4400-4700 \\
5100-5400\end{array}$ & $0.16 \pm 0.02$ & $1.71 \pm 0.06$ \\
\hline \multirow[t]{2}{*}{ PG 1310-108 } & $U_{s}$ & $3500-3600$ & $0.13 \pm 0.02$ & $1.85 \pm 0.03$ \\
\hline & $\mathrm{H} \beta$ & $4787-4932$ & $0.05 \pm 0.01$ & $1.29 \pm 0.02$ \\
\hline \multirow[t]{2}{*}{ Zw 229-015 } & $U_{s}$ & $3500-3570$ & $0.25 \pm 0.03$ & $2.65 \pm 0.06$ \\
\hline & $\mathrm{H} \alpha$ & $6470-6664$ & $0.13 \pm 0.02$ & $1.54 \pm 0.02$ \\
\hline
\end{tabular}

Table 3

(Continued)

\begin{tabular}{ccccc}
\hline \hline Galaxy & $\begin{array}{l}\text { Light } \\
\text { Curve }\end{array}$ & $\begin{array}{l}\text { Rest Wave- } \\
\text { lengths }(\AA)\end{array}$ & $F_{\text {var }}$ & $R_{\max }$ \\
\hline & $\mathrm{H} \beta$ & $4767-4912$ & $0.25 \pm 0.03$ & $2.81 \pm 0.07$ \\
& $\mathrm{H} \gamma$ & $4281-4388$ & $0.27 \pm 0.04$ & $2.82 \pm 0.13$ \\
\hline
\end{tabular}

Note. These measurements include the constant narrow-line component contributions to the Balmer-line and $\mathrm{He}$ in light curves, and the constant hostgalaxy contribution to the $U_{s}$ light curve. The wavelength column lists the range over which flux density was integrated for the light curve measurement. As described in the text, the $\mathrm{H} \beta, \mathrm{H} \gamma, \mathrm{He}$ II, and Fe II light curves were measured from individual components of the decomposed blue-side data, while $U_{s}, \mathrm{H} \alpha$, and $\mathrm{H} \delta$ were measured from the total-flux spectra.

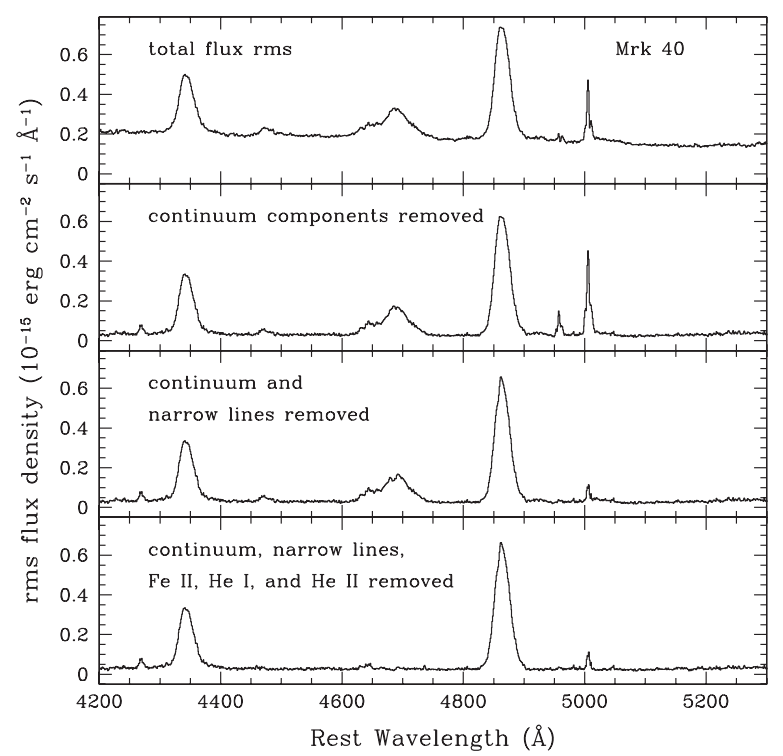

Figure 6. Different versions of the rms spectrum for Mrk 40. The top panel shows the standard rms spectrum constructed using the nightly total flux spectra. The second panel shows the rms after removing the AGN continuum and host-galaxy starlight components from each nightly spectrum. In the third panel, narrow emission lines have also been removed ([O III] $\lambda \lambda 44959,5007$, $\mathrm{H} \beta$, and $\mathrm{He}$ II), and in the bottom panel, the broad $\mathrm{He}$ II and $\mathrm{Fe}$ II components are also removed from the nightly spectra. This last version of the rms spectrum, with all model components removed other than broad $\mathrm{H} \beta$, is the one used to measure the width of broad $\mathrm{H} \beta$ in the rms spectrum.

because $R_{\max }$ is particularly sensitive to outlier values at the highest and lowest fluxes.

Emission-line light curves having $F_{\mathrm{var}}>0.1$ correspond to strong variability from which lags can generally be measured well. In our sample, the objects exceeding this threshold for $\mathrm{H} \beta$ variations are Mrk 40, Mrk 50, Mrk 1511, NGC 4593, and $\mathrm{Zw}$ 229-015. For these AGNs, we plot light curves of the $U_{s}$ flux density, the Balmer lines, and also $\mathrm{He}$ II and $\mathrm{Fe}_{\text {II }}$ in those cases where there is some distinct variability above the level of the noise for these components. We also plot multi-line light curves for Mrk 279 and Mrk 704. Mrk 279 has a lower $\mathrm{H} \beta$ variability amplitude of $F_{\mathrm{var}}=0.07$, but its light curve exhibits distinct up-and-down variations which provide useful diagnostics of the emission-line lags. Mrk 704 exhibits a downward trend in the continuum and emission-line light 


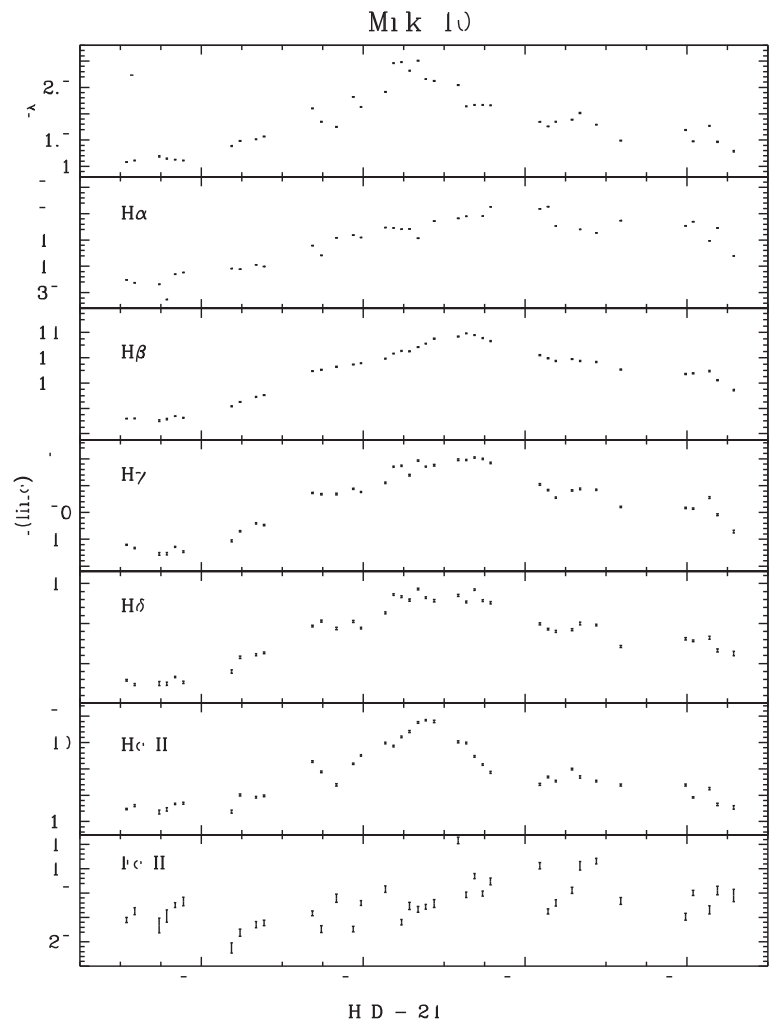

Figure 7. Light curves of Mrk 40. The $y$-axis units for this and subsequent light curve plots are $10^{-15} \mathrm{erg} \mathrm{cm}^{-2} \mathrm{~s}^{-1} \AA^{-1}$ for the $U_{s}$-band continuum flux density and $10^{-15} \mathrm{erg} \mathrm{cm}^{-2} \mathrm{~s}^{-1}$ for the emission-line fluxes. Plotted error bars represent the propagated uncertainties from the spectral extractions only, as listed in Table 4, and do not incorporate the estimated residual flux normalization scatter as determined from the $[\mathrm{O}$ III] emission line.

curves, but the nearly monotonic trend contains little shorttimescale structure and is not optimal for determination of lags. For the remaining objects in the sample, all of which have $F_{\mathrm{var}}$ $(\mathrm{H} \beta)<0.1$, light curves are shown only for $U_{s}$ and $\mathrm{H} \beta$ in Figure 14. Aside from $\mathrm{H} \beta$, the light curves for other emission lines in these objects are too noisy to be of much use. A few of these AGNs, such as Mrk 141, Mrk 493, and PG 1310-108, show distinct features in their light curves which may provide some limited information on the $\mathrm{H} \beta$ lag. Table 3 lists the lightcurve statistics $F_{\mathrm{var}}$ and $R_{\max }$ for each light curve displayed in Figures 7-14. For completeness, we list statistics measured for the $U_{s}$ continuum light curves for each AGN, including the low-variability objects shown in Figure 14.

Table 2 also presents measurements of $f_{\lambda}(5100 \AA)$, the AGN continuum flux density at $5100 \AA$ in the AGN rest frame. This quantity is most often used to determine AGN luminosities for use in the radius-luminosity relationship (e.g., Bentz et al. 2009a). This wavelength corresponds to a local minimum in Fe II flux, making it a good choice for measurement of the featureless continuum level in total-flux spectra. We used the AGN power-law component of the fit to the mean spectrum to measure $f_{\lambda}(5100 \AA)$ for each AGN. The formal fitting uncertainty on the power-law component flux for the mean spectrum is typically very small (a few percent) and not a good measure of the actual uncertainty. From examination of the fitting results using all three $\mathrm{Fe}$ II templates, we find that the rms scatter among the power-law component fluxes at $5100 \AA$ for the three fits ranges from 4 to $13 \%$ of the mean value. For uniformity, we adopt an estimated $10 \%$ uncertainty on the

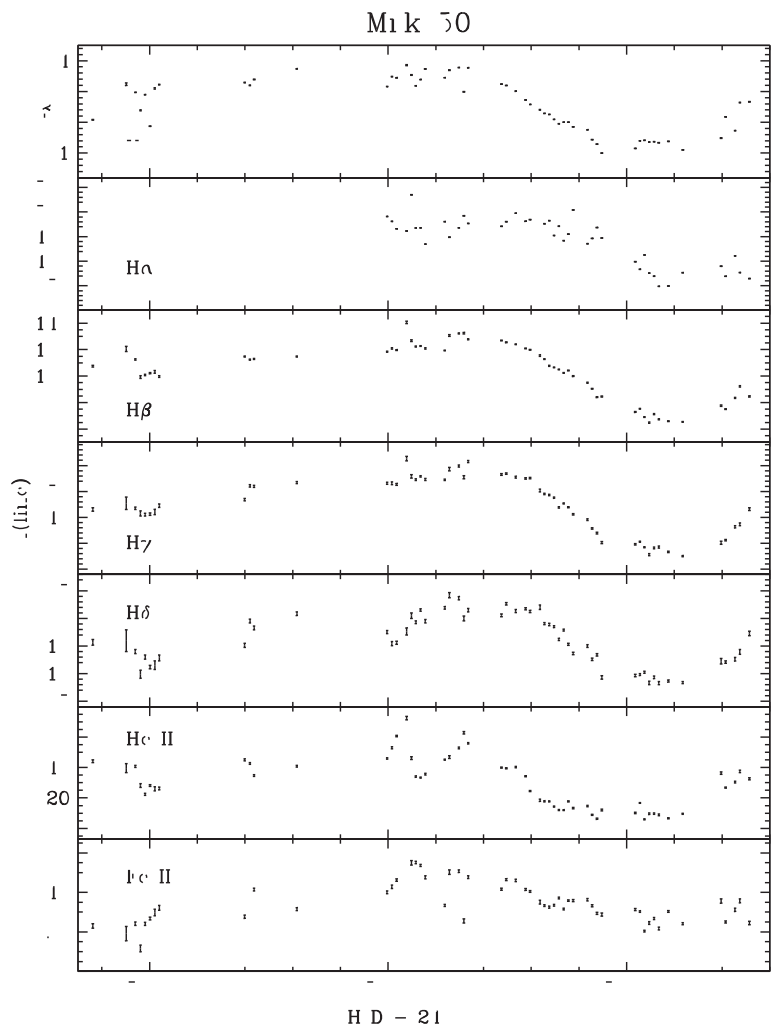

Figure 8. Light curves of Mrk 50, including observations taken during January-March before the start of the main campaign.

$5100 \AA$ f featureless continuum flux at this wavelength resulting from differences among the $\mathrm{Fe}$ II templates. We also combine with this (by addition in quadrature) an estimate of the overall photometric uncertainty on the continuum flux density given by $\delta\left(\left[\mathrm{O}_{\mathrm{III}}\right]\right) / f\left(\left[\mathrm{O}_{\mathrm{III}}\right]\right) \times f_{\lambda}(5100 \AA)$, where the $\left[\mathrm{O}_{\mathrm{III}}\right]$ flux and uncertainty values are listed in Table 2 . Improved values of $f_{\lambda}(5100 \AA)$ can be determined using $H S T V$-band images to remove the host-galaxy contribution more accurately from the total spectroscopic flux density. ${ }^{32}$

For the strongly variable AGNs, we generally find higher values of $F_{\mathrm{var}}$ for the higher-order Balmer lines: $F_{\mathrm{var}}(\mathrm{H} \delta)>F_{\mathrm{var}}$ $(\mathrm{H} \gamma)>F_{\mathrm{var}}(\mathrm{H} \beta)>F_{\mathrm{var}}(\mathrm{H} \alpha)$. This overall trend is consistent with results from previous work (e.g., Bentz et al. 2010a), and is in accord with expectations from photoionization modeling (Korista \& Goad 2004). The He II $\lambda 4686$ line is by far the most strongly variable line in the optical spectrum, following the trend first seen in NGC 5548 by Peterson \& Ferland (1986). For the six objects having $\mathrm{He}$ II light curves, the ratio $F_{\mathrm{var}}$ $(\mathrm{He}$ II $) / F_{\text {var }}(\mathrm{H} \beta)$ has a mean value of 3.7 . The strong responsivity and prompt response of $\mathrm{He}$ II is easily visible in the light curves: for example, in Mrk 40, the He II emission drops very steeply following the decline in continuum luminosity at about HJD 5685, while the decline in the Balmer-line fluxes is much shallower and more gradual (Figure 7).

While it is possible to measure reverberation lags based on the data presented here for the highly variable AGNs in our sample, we find that for some objects the cross-correlation

\footnotetext{
32 New images of Mrk 50, Mrk 704, Mrk 1511, and Zw 229-015 will be obtained with WFC 3 in the F547M filter as part of HST Cycle 22 program GO13816 (PI: Bentz) along with 10 other recently reverberation-mapped AGNs compiled from other programs.
} 


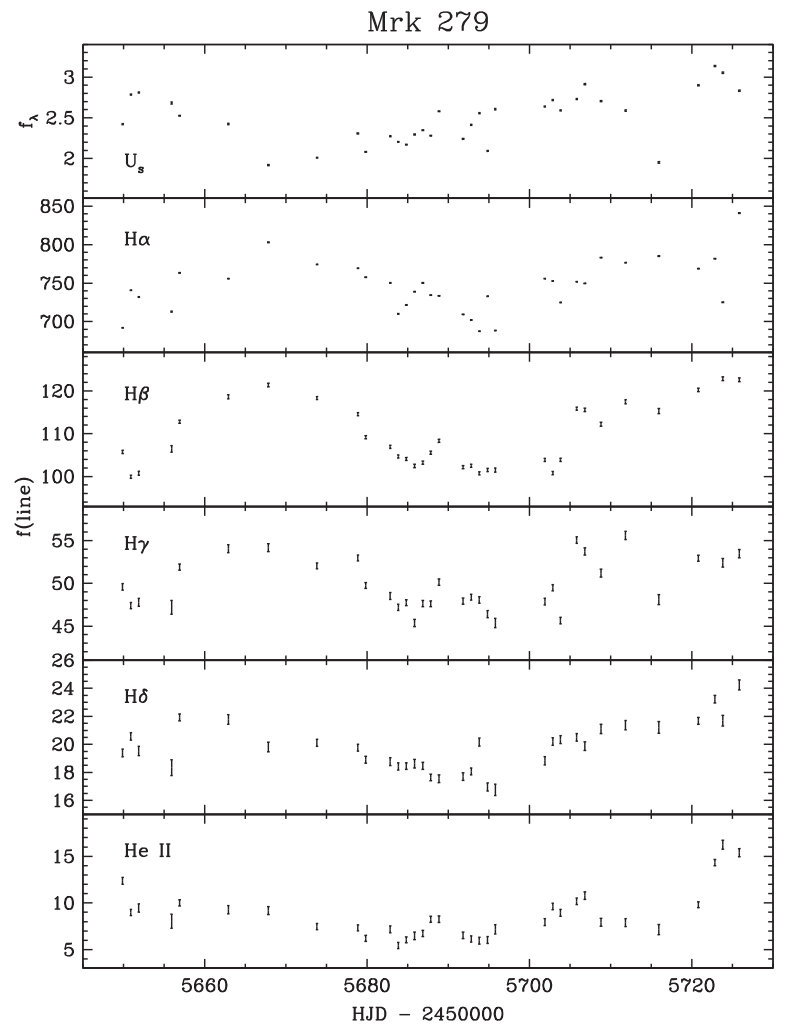

Figure 9. Light curves of Mrk 279.

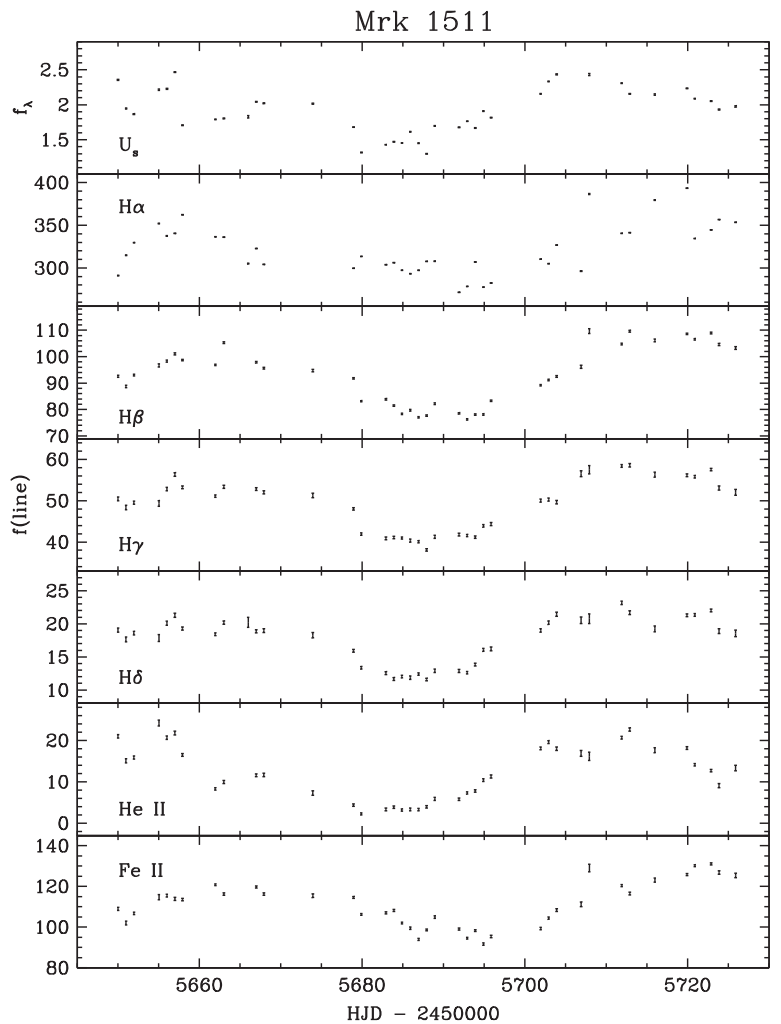

Figure 10. Light curves of Mrk 1511.

measurements show evidence for an excess and spurious signal at zero lag, caused by correlated flux-calibration errors in the continuum and emission-line light curves. Thus, we defer the measurement of lags to a later paper in this series, in which our

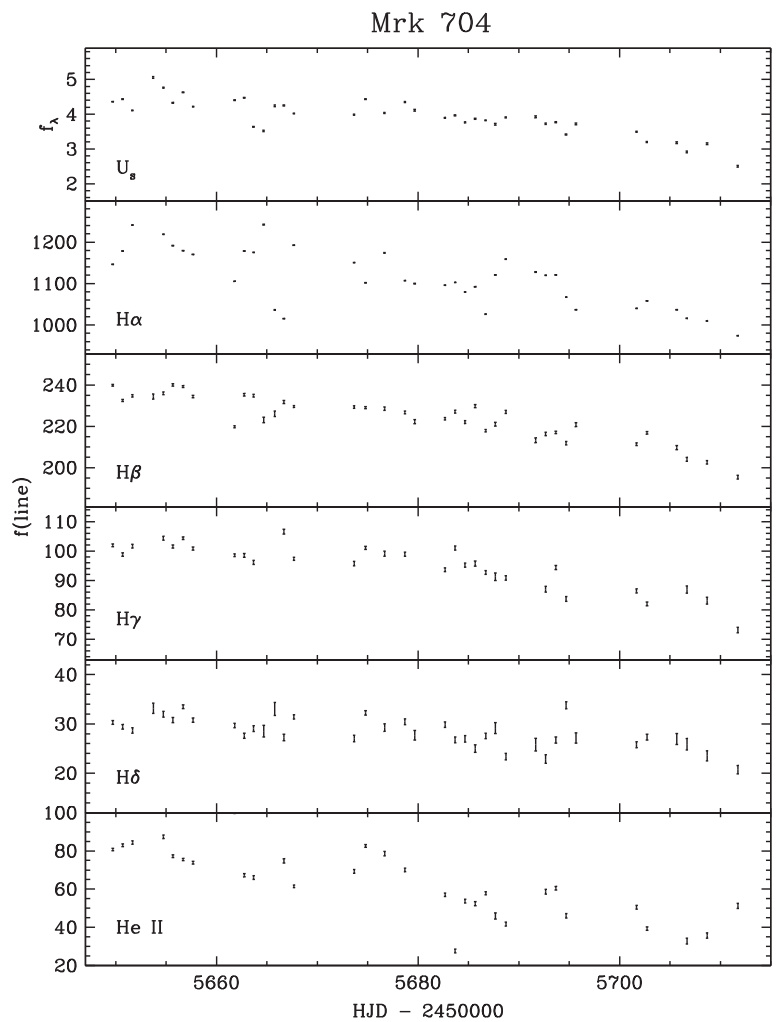

Figure 11. Light curves of Mrk 704.

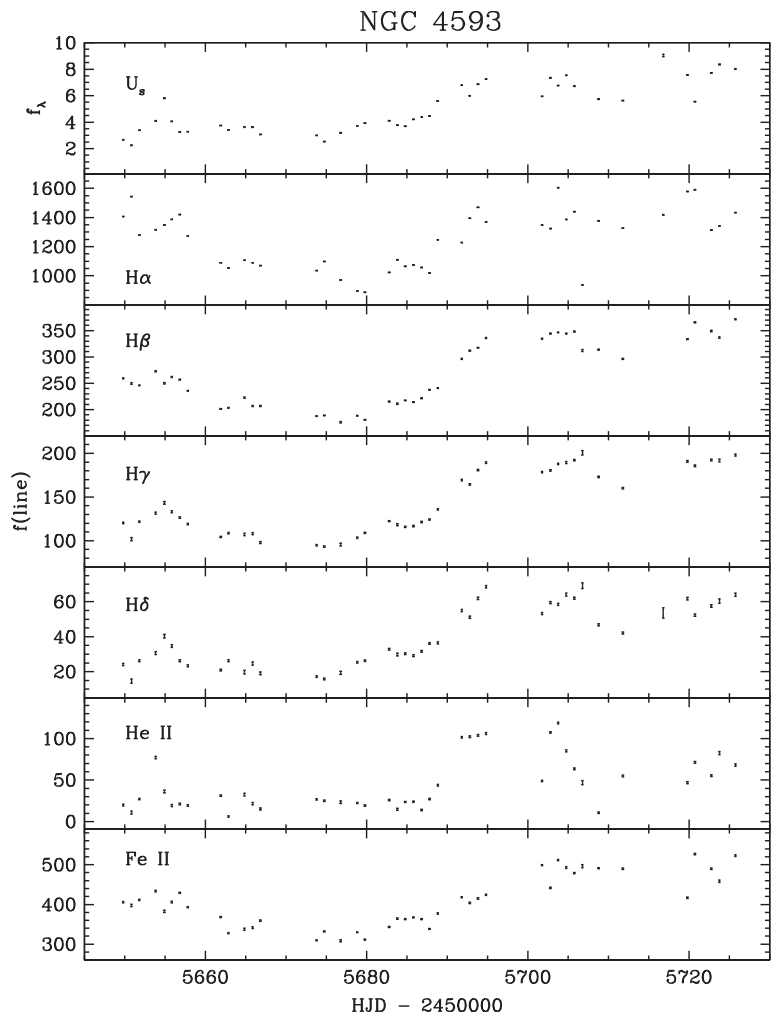

Figure 12. Light curves of NGC 4593.

emission-line light curves will be combined with $V$-band light curves which will be presented separately. The $V$-band light curves generally have a higher cadence and higher $\mathrm{S} / \mathrm{N}$ than the $U_{s}$ light curves. Determination of virial masses for the black 


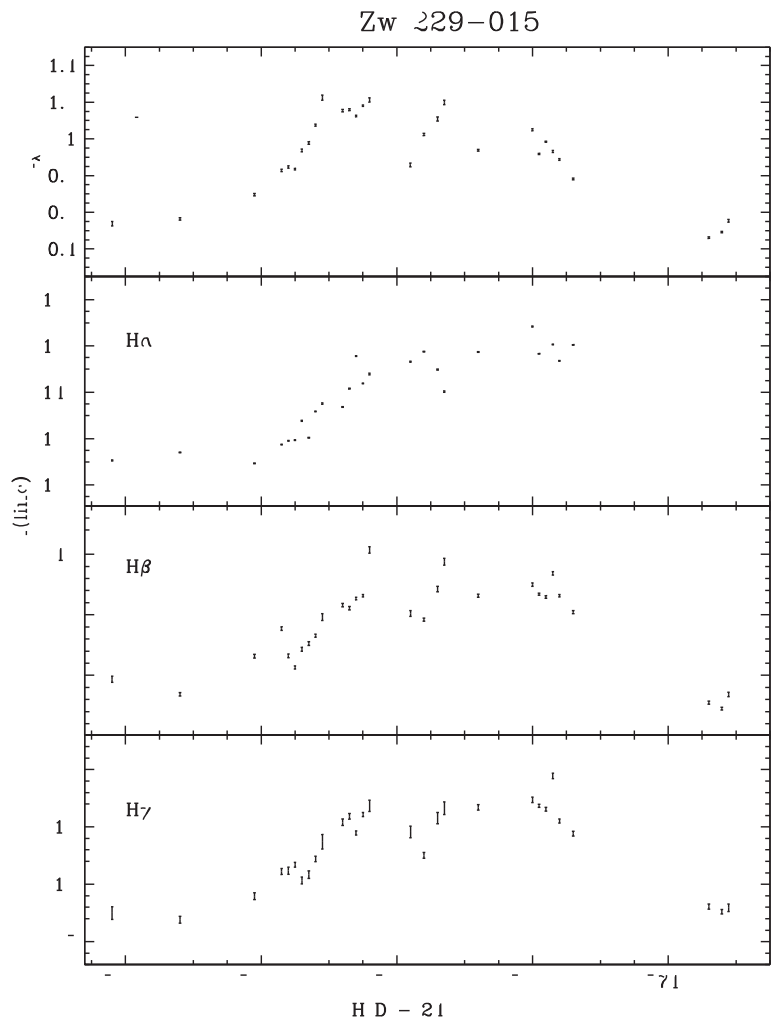

Figure 13. Light curves of $\mathrm{Zw} 229-015$, including the three data points taken after the end of the main campaign.

holes in these AGNs will be done using lags measured against the $V$-band light curves.

\section{BROAD H $\beta$ WIDTHS AND VELOCITY SHIFTS}

\subsection{Instrumental Resolution}

To quantify the broad-line widths, it is necessary to correct measurements for the instrumental broadening in observations with the $4^{\prime \prime}$-wide slit. The spectral resolution for observations of the intrinsically compact BLR as observed through a $4^{\prime \prime}$ slit will be seeing-dependent, and will vary slightly from night to night due to variations in spectrograph focus. However, the impact of instrumental broadening on the broad emission lines in these AGNs is very small, and we use a single representative value for the instrumental broadening for simplicity. For the blue-side data we follow the same method used by Bentz et al. (2009b) and Barth et al. (2011a) to estimate the instrumental FHWM by using observations of an AGN and an arc lamp in $2^{\prime \prime}$ and $4^{\prime \prime}$ slit widths. Mrk 40 was observed on the night of 2011 May 4 in both of these slit widths. For the spectral region around $\mathrm{H} \beta$, we use the $\operatorname{Cd}{ }_{\mathrm{I}} \lambda 5085 \AA$ line as the nearest reference calibration line. From the $2^{\prime \prime}$ observations of the AGN and the comparison lamp, we subtract the width of the $\mathrm{Cd}$ I line in quadrature from the observed [O III] width to derive an estimate of the intrinsic $[\mathrm{O}$ III] line width of FWHM $\approx 4.9 \AA$. Then, the instrumental broadening for the $4^{\prime \prime}$ slit is taken to be the difference in quadrature between the $[\mathrm{O}$ III] line width as observed through the $4^{\prime \prime}$ slit $(\mathrm{FWHM}=8.1 \AA)$ and the intrinsic [O III] width. This gives an instrumental FWHM of $6.45 \AA$ or $380 \mathrm{~km} \mathrm{~s}^{-1}$, and an instrumental dispersion of $\sigma_{\text {inst }} \approx$ $162 \mathrm{~km} \mathrm{~s}^{-1}$.
For the red-side data, there is no strong narrow line that can be used as a reference for comparison between the $2^{\prime \prime}$ and $4^{\prime \prime}$ observations, and instead we use the widths of comparison lamp lines as observed through the $4^{\prime \prime}$ slit as a rough estimate of the instrumental broadening, obtaining an instrumental FWHM of $7.7 \AA$ or $\sim 330 \mathrm{~km} \mathrm{~s}^{-1}$ for the region around the $\mathrm{H} \alpha$ line. These instrumental widths are much smaller than the broad emission-line widths in the AGNs, and the corrections to the observed line widths are very small.

\subsection{Broad $H \beta$ Widths in Mean and rms Spectra}

The width of the broad $\mathrm{H} \beta$ line is a primary quantity used for determination of $\mathrm{BH}$ masses from reverberation data, and it is of interest to measure both the FWHM and the dispersion (or second moment) of the $\mathrm{H} \beta$ line profile in both the mean and the rms spectra when possible. We use the results of our spectral decompositions to measure these width parameters for the broad $\mathrm{H} \beta$ line after isolating it from the contributions of other emission-line and continuum components. For each nightly spectrum, we subtract all model components other than broad $\mathrm{H} \beta$ from the data, leaving the broad $\mathrm{H} \beta$ profile as the residual. From this time series of residuals, we construct mean and rms spectra, and measure widths empirically from the mean and rms line profiles as described below. The mean spectrum has a continuum level of zero (modulated by residual noise from the spectral decompositions), while the continuum level in the rms spectrum is nonzero due to photon-counting noise and residual errors in the nightly spectral decompositions. This noise continuum level in the rms spectra is generally flat and featureless outside the $\mathrm{H} \beta$ profile (as seen in the bottom panel of Figure 6) and can be subtracted off with a simple linear fit to regions on either side of the line. For each AGN we selected broad continuum windows redward and blueward of the $\mathrm{H} \beta$ line to fit and subtracted this continuum from the rms spectrum, avoiding any residual spikes at the wavelengths of [O III] or other strong features.

Line width parameters were measured following methods similar to those of Peterson et al. (2004). The profile FWHM was measured by starting at the profile peak and moving redward until reaching a flux equal to half the peak value (using linear interpolation between pixels on the profile shoulder to determine the wavelength at half maximum), and similarly moving blueward until reaching half of the peak flux, and determining the separation in Angstroms between the two half-peak points. To measure the line dispersion $\sigma_{\text {line }}$ for either the mean or rms profile, we first determine the line centroid wavelength $\lambda_{0}$ :

$$
\lambda_{0}=\frac{\sum \lambda_{i} f_{i}}{\sum f_{i}},
$$

where $f_{i}$ is the flux density at pixel $i$ and the summation is carried out over all pixels in the profile. Then, the line dispersion is calculated according to

$$
\sigma_{\text {line }}^{2}=\left(\frac{\sum \lambda_{i}^{2} f_{i}}{\sum f_{i}}\right)-\lambda_{0}^{2} .
$$

The endpoints of the summation region are determined visually for each AGN by identifying the location where the line profile meets the surrounding continuum level. 

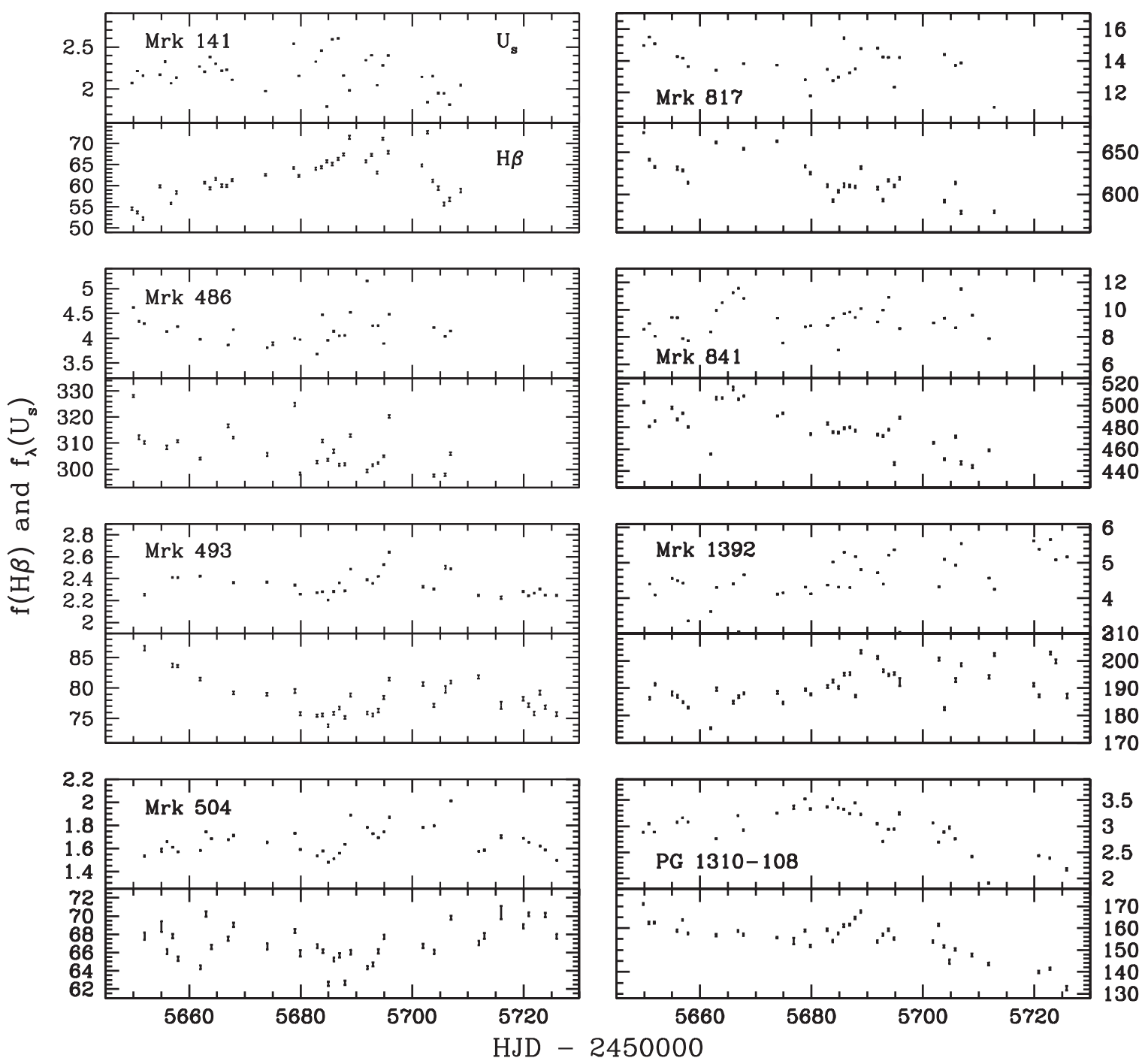

Figure 14. Light curves of the remaining objects in the sample. In each panel, the upper and lower subpanels show the $U_{s}$ and $\mathrm{H} \beta$ light curves, respectively.

In order to assess the uncertainty in the measured width parameters, we carry out a Monte Carlo procedure, following Peterson et al. (2004) and Bentz et al. (2009b), by creating $10^{4}$ modified realizations of the mean and rms profiles and measuring the width parameters for each one. Each realization is constructed from the time series of spectra by randomly selecting $N$ individual nightly spectra (where $N$ is the total number of spectra in the time series), allowing duplications, calculating the mean and rms from this revised set of spectra, and measuring the broad $\mathrm{H} \beta$ widths from each. For measurements of the rms profile, we allow the endpoints of the continuum fitting region in each realization to vary randomly within a range of $\pm 10 \AA$ from our initial selections, and for measurements of line dispersions from either the mean or rms profiles, we allow the endpoints of the summation region in each realization to vary randomly within $\pm 10 \AA$ from our initial selections. The final line width and uncertainty are taken to be the mean and standard deviation of the distribution of measurements over these $10^{4}$ iterations.

Finally, the line widths are converted to velocity units, and the instrumental FWHM or $\sigma$ are subtracted in quadrature from the measured FWHM or $\sigma$ values (respectively) to correct for
Table 4

Light Curve Data

\begin{tabular}{ccccc}
\hline \hline Galaxy & Light Curve & HJD & $f$ & $\delta_{f}$ \\
\hline Mrk 40 & $U_{s}$ & 5650.75 & 1.081 & 0.004 \\
& 5651.75 & 1.112 & 0.006 \\
& 5654.80 & 1.191 & 0.013 \\
& 5655.73 & 1.146 & 0.010 \\
& 5656.76 & 1.127 & 0.004 \\
& 5657.77 & 1.111 & 0.007 \\
& 5663.74 & 1.386 & 0.009 \\
& 5664.78 & 1.481 & 0.006 \\
& 5666.76 & 1.515 & 0.005 \\
& 5667.75 & 1.567 & 0.005 \\
& 5673.73 & 2.101 & 0.006 \\
& 5674.82 & 1.848 & 0.006 \\
\hline
\end{tabular}

Note. Dates are listed as HJD—2450000. Units are $10^{-15} \mathrm{erg} \mathrm{cm}^{-2} \mathrm{~s}^{-1} \AA^{-1}$ for $U_{s}$, and $10^{-15} \mathrm{erg} \mathrm{cm}^{-2} \mathrm{~s}^{-1}$ for emission-line light curves. The listed uncertainties $\delta_{f}$ correspond to the propagated uncertainties from photon counting and detector readout noise, and do not include the additional contribution from residual flux-scaling errors.

(This table is available in its entirety in machine-readable form.) 
Table 5

Broad $\mathrm{H} \beta$ Component Widths in Mean and rms Spectra

\begin{tabular}{llccc}
\hline \hline Galaxy & \multicolumn{2}{c}{ Mean Spectrum } & \multicolumn{2}{c}{ rms Spectrum } \\
& $\begin{array}{c}\text { FWHM } \\
\left(\mathrm{km} \mathrm{s}^{-1}\right)\end{array}$ & $\begin{array}{c}\sigma_{\text {line }} \\
\left(\mathrm{km} \mathrm{s}^{-1}\right)\end{array}$ & $\begin{array}{c}\text { FWHM } \\
\left(\mathrm{km} \mathrm{s}^{-1}\right)\end{array}$ & $\begin{array}{c}\sigma_{\text {line }} \\
\left(\mathrm{km} \mathrm{s}^{-1}\right)\end{array}$ \\
\hline Mrk 40 & $2021 \pm 17$ & $1058 \pm 9$ & $1688 \pm 26$ & $740 \pm 17$ \\
Mrk 50 & $4101 \pm 56$ & $2024 \pm 31$ & $3355 \pm 128$ & $2020 \pm 103$ \\
Mrk 141 & $5129 \pm 45$ & $2280 \pm 21$ & $\ldots$ & $\ldots$ \\
Mrk 279 & $4099 \pm 43$ & $1821 \pm 13$ & $3306 \pm 338$ & $1778 \pm 71$ \\
Mrk 486 & $2106 \pm 8$ & $1332 \pm 14$ & $\ldots$ & $\ldots$ \\
Mrk 493 & $1717 \pm 25$ & $1128 \pm 41$ & $\ldots$ & $\ldots$ \\
Mrk 504 & $1927 \pm 7$ & $1058 \pm 14$ & $\ldots$ & $\ldots$ \\
Mrk 704 & $6664 \pm 66$ & $2911 \pm 61$ & $\ldots$ & $\ldots$ \\
Mrk 817 & $5593 \pm 32$ & $2718 \pm 45$ & $\ldots$ & $\ldots$ \\
Mrk 841 & $6065 \pm 34$ & $2794 \pm 48$ & $\ldots$ & $\ldots$ \\
Mrk 1392 & $5421 \pm 31$ & $2103 \pm 17$ & $\ldots$ & $\ldots$ \\
Mrk 1511 & $4154 \pm 28$ & $1828 \pm 12$ & $3236 \pm 65$ & $1506 \pm 42$ \\
NGC 4593 & $4264 \pm 41$ & $1925 \pm 38$ & $3597 \pm 72$ & $1601 \pm 40$ \\
PG 1310-108 & $3422 \pm 21$ & $1823 \pm 20$ & $\ldots$ & $\ldots$ \\
Zw 229-015 & $3705 \pm 203$ & $1747 \pm 56$ & $1789 \pm 93$ & $1609 \pm 109$ \\
\hline
\end{tabular}

Note. Widths are measured from the mean and $\mathrm{rms}$ of the broad $\mathrm{H} \beta$ component spectra, as described in the text.

instrumental broadening. The results of these measurements are reported in Table 5. Width parameters for the rms profile are only reported for the five AGNs having $F_{\mathrm{var}}(\mathrm{H} \beta)>0.1$ and for Mrk 279. For the remainder of the sample, the rms spectra contain very little flux as a result of the overall low variability amplitude, and the Monte Carlo error analysis procedure produces many iterations in which the FWHM or $\sigma_{\text {line }}$ are not well-defined. Widths measured from the rms profiles for these low-variability AGNs have large uncertainties and would not be particularly useful for deriving estimates of black hole masses, despite the fact that some of these low-variability AGNs appear to show clear signal at $\mathrm{H} \beta$ in the rms spectra (Figure 5).

An important caveat for the line width measurements, particularly for the line dispersions, is that the red wing of $\mathrm{H} \beta$ is often blended with a substantial contribution of Fe II and possibly $\mathrm{He}_{\mathrm{I}}$ as well. As described above, the exact amount of this contamination is difficult to determine, and the endpoint of the red wing of the line is usually not well determined. Since the line dispersion values are highly sensitive to the amount of flux in the line wings, broad $\mathrm{H} \beta$ line dispersions are particularly susceptible to systematic errors resulting from this deblending. We note that in most past reverberation-mapping work, no deblending was attempted at all, potentially leading to overestimates of $\mathrm{H} \beta$ line dispersions. The FWHM may be modestly affected by this red-wing contamination as well.

\subsection{Broad $H \beta$ Width and Velocity Centroid Variations}

Width parameters (FWHM and $\sigma_{\text {line }}$ ) were also measured from each nightly spectrum for the high-variability objects in order to search for time-dependent changes in profile width, following the same methods described above. These widths were measured from the spectra of the broad $\mathrm{H} \beta$ component alone, constructed by subtracting all other model components (including the narrow $\mathrm{H} \beta$ component) from each total flux spectrum. To assess the uncertainty in FWHM and $\sigma_{\text {line }}$, we created 1000 modified realizations of each spectrum by

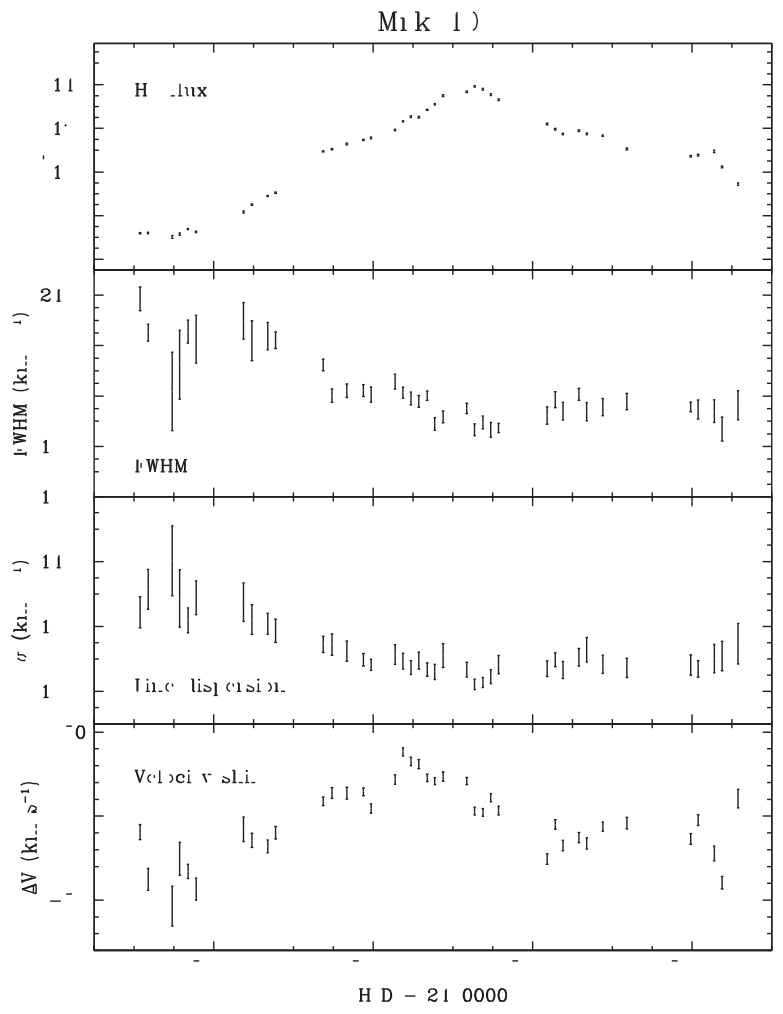

Figure 15. Broad $\mathrm{H} \beta$ parameters as a function of time for Mrk 40. The top panel shows the same $\mathrm{H} \beta$ light curve as in Figure 7, in units of $10^{-15} \mathrm{erg} \mathrm{cm}^{-2}$ $\mathrm{s}^{-1}$. Middle panels show the broad $\mathrm{H} \beta \mathrm{FWHM}$ and line dispersion $\sigma_{\text {line }}$, and the bottom panel shows the velocity shift $\Delta V$ measured relative to the mean spectrum.

adding Gaussian random noise with the noise dispersion equal to the amplitude of the error spectrum at each wavelength, and then measuring the FWHM and $\sigma_{\text {line }}$ from each modified spectrum. The uncertainties on FWHM and $\sigma_{\text {line }}$ were taken to be the standard deviations of the distributions of values measured from this procedure. In general, the line widths show an anticorrelation with flux; Section 7 discusses this trend further.

Using the same spectra of the broad $\mathrm{H} \beta$ component, we measured the broad-line velocity centroid shift relative to the broad $\mathrm{H} \beta$ component of the mean spectrum, using a crosscorrelation method based on the techniques described by Shen et al. (2013) and Ju et al. (2013) in the context of searching for binary black hole orbital motion in quasars. The wavelength centroid of the broad $\mathrm{H} \beta$ component is a parameter determined by our spectral fitting procedure, but the fitted line centroid can be strongly influenced by asymmetric wings on the line profile, particularly in the $\mathrm{H} \beta$ red shelf. In contrast, the crosscorrelation line shift as measured by Shen et al. (2013) and $\mathrm{Ju}$ et al. (2013) is a better measure of an overall wavelength shift of the line as a whole, and is less sensitive to noise in the high-velocity wings. The velocity shifts are discussed in Section 8.

The nightly width and velocity shift parameters are displayed in Figures 15-19 for Mrk 40, Mrk 50, Mrk 279, Mrk 1511, and NGC 4593, all of which show distinct time-dependent trends in line profile width and/or velocity. For the remainder of the sample, no clear signal was detected beyond random scatter in the measurements. 


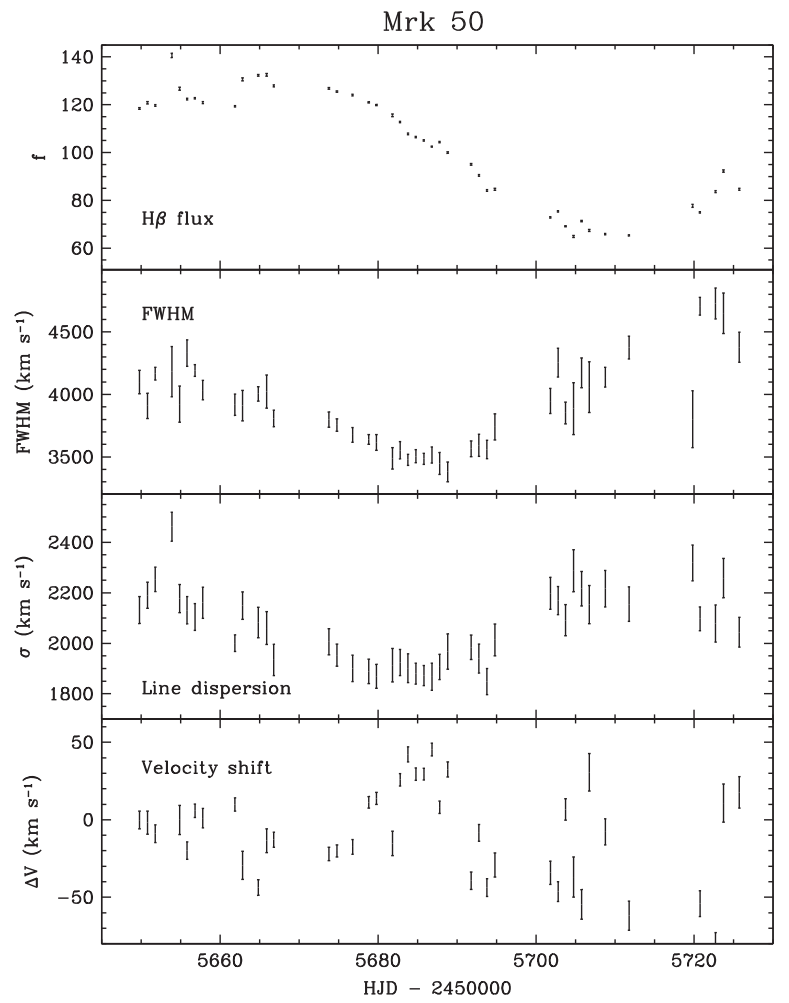

Figure 16. Broad $\mathrm{H} \beta$ parameters for Mrk 50. Only the time span of the main campaign is shown, because the data points from earlier observations are very noisy.

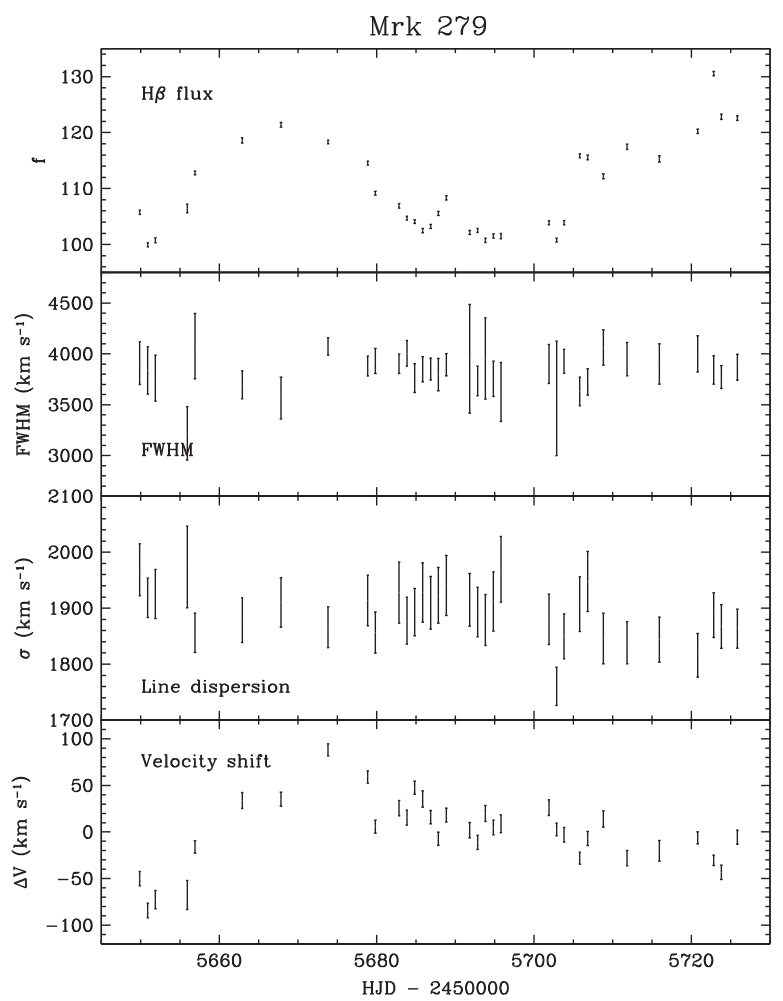

Figure 17. Broad $\mathrm{H} \beta$ parameters for Mrk 279. The nearly constant $\mathrm{H} \beta$ width for this AGN is an artifact of the constraint that had to be imposed in order to prevent the line width from reaching unphysically large values in the fits.

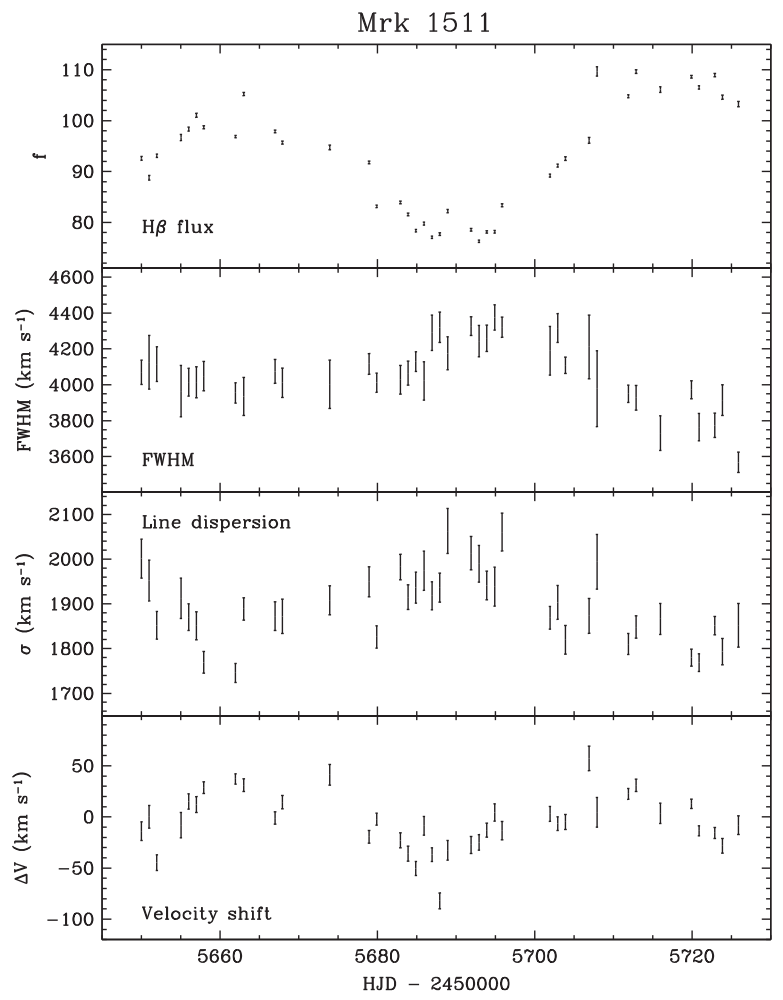

Figure 18. Broad $\mathrm{H} \beta$ parameters for Mrk 1511.

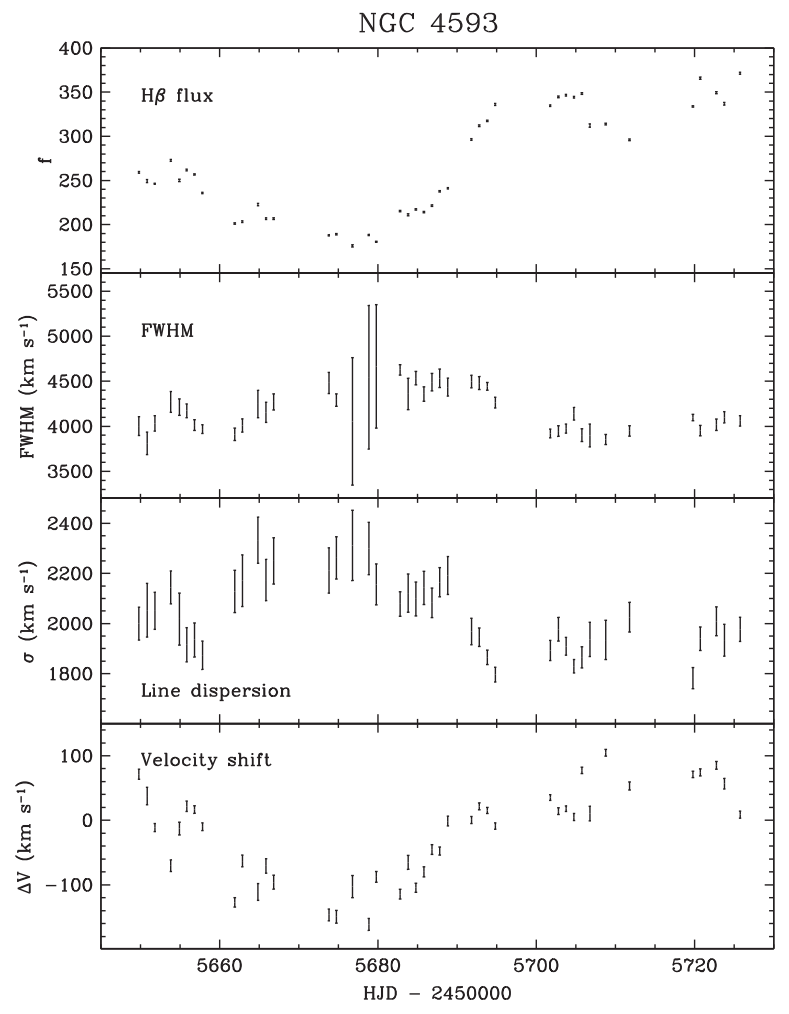

Figure 19. Broad $\mathrm{H} \beta$ parameters for NGC 4593. 


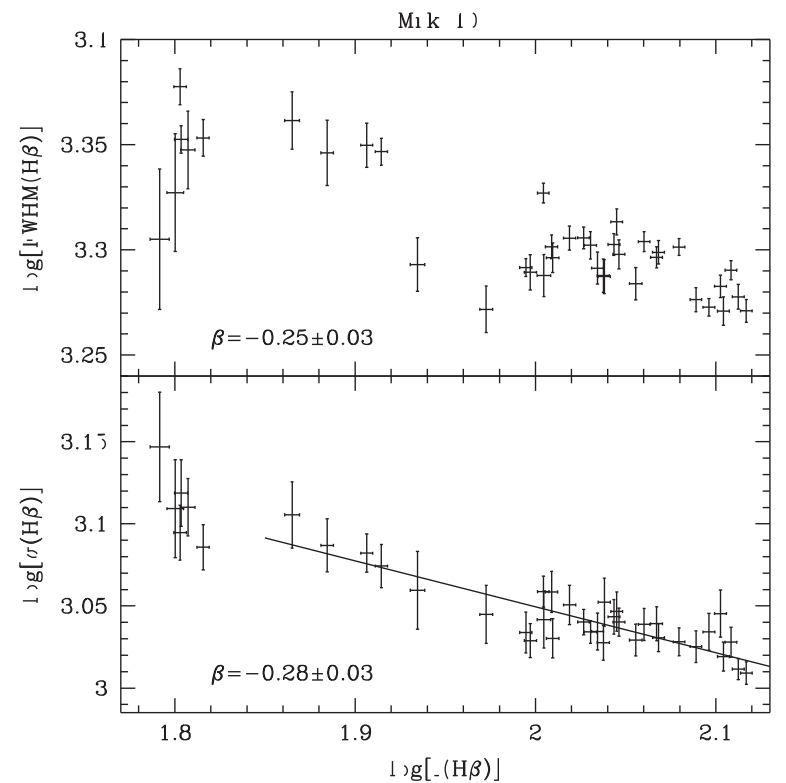

Figure 20. Relationship between broad $\mathrm{H} \beta$ width and flux for Mrk 40. In each panel, the solid line shows the best fit to the relationship with slope $\beta$. Line widths (FWHM and $\sigma_{\text {line }}$ ) are in $\mathrm{km} \mathrm{s}^{-1}$, and flux units are $10^{-15} \mathrm{erg} \mathrm{cm}^{-2} \mathrm{~s}^{-1}$.

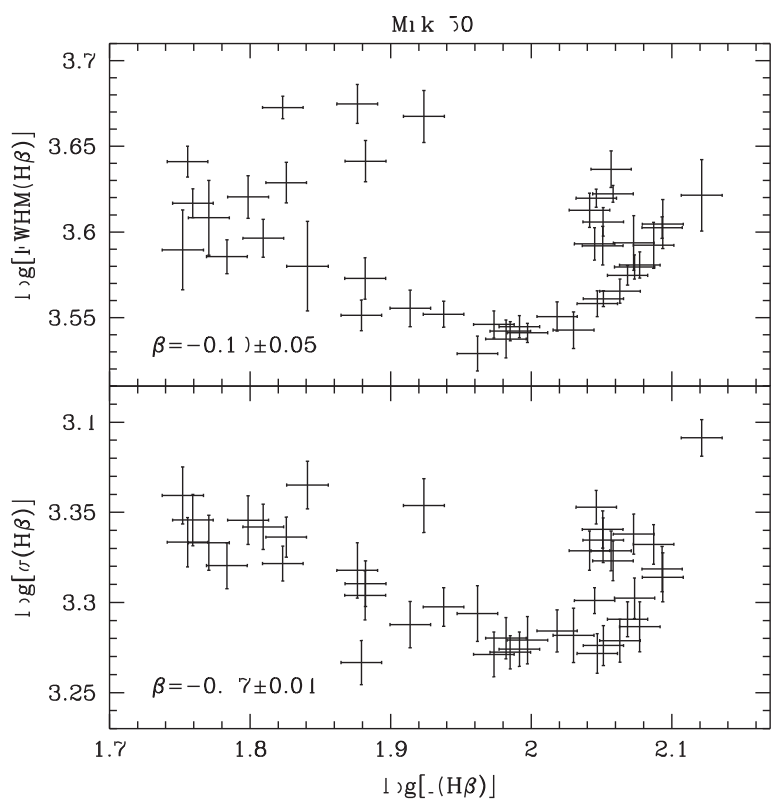

Figure 21. Relationship between broad $\mathrm{H} \beta$ width and flux for Mrk 50. Only data points from the main campaign are included.

\section{RELATIONSHIPS BETWEEN BROAD-LINE WIDTH AND LUMINOSITY}

Figures 20-23 display the relationship between broad $\mathrm{H} \beta$ width (FWHM or $\sigma_{\text {line }}$ ) and broad $\mathrm{H} \beta$ flux. The narrow $\mathrm{H} \beta$ component flux from the mean spectrum decomposition has been subtracted from the total $\mathrm{H} \beta$ light curve in each case, to obtain a light curve of the broad $\mathrm{H} \beta$ line alone. The flux uncertainties have been expanded by including the estimated uncertainty in spectral scaling determined from the [O III] excess scatter, using the results listed in Table 2 . In the case of Mrk 50 (Figure 21) we include only data points from the main observing campaign, since the observations taken prior to the start of the campaign are much noisier. Mrk 279 is excluded

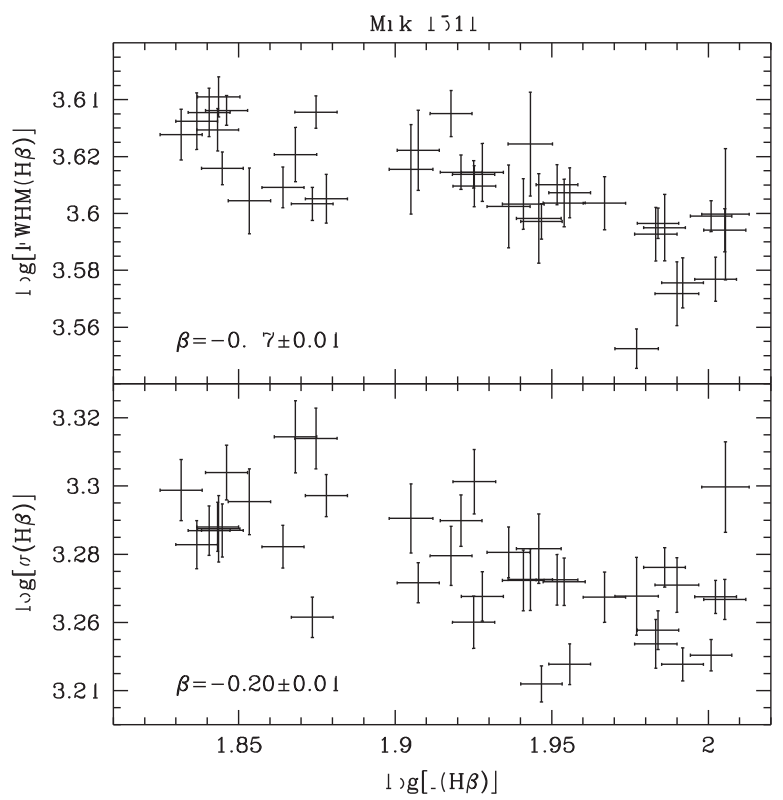

Figure 22. Relationship between broad $\mathrm{H} \beta$ width and flux for Mrk 1511 .

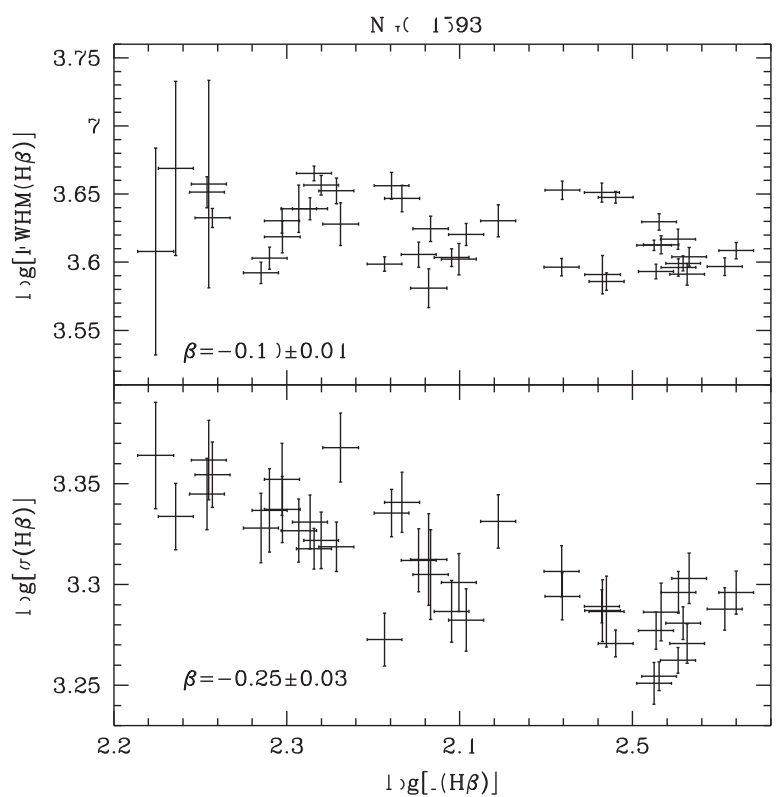

Figure 23. Relationship between broad $\mathrm{H} \beta$ width and flux for NGC 4593 .

since the broad $\mathrm{H} \beta$ width could not be measured accurately, as described previously.

Although there is substantial scatter in some of the plots, in each case we find an overall anticorrelation between the broad $\mathrm{H} \beta$ width and flux. To quantify this relationship, we use the Bayesian linmix err regression method of Kelly (2007), fitting a model of the form $\log [\Delta V(\mathrm{H} \beta)]=$ $\beta \log [f(\mathrm{H} \beta)]+\alpha$, where $\Delta V$ represents either FWHM or $\sigma_{\text {line }}$. The slopes determined by the linmix_err regression are shown in each figure and range from $\beta=-0.07$ to -0.28 .

Similar trends between broad $\mathrm{H} \beta$ width and luminosity have been seen previously in high-quality reverberation mapping datasets. Examples include NGC 5548 (Denney et al. 2009a) and Mrk 40 (Arp 151; Park et al. 2012a). The anticorrelation between broad $\mathrm{H} \beta$ width and luminosity arises as a result of the relationship between the ionizing flux and the local 
responsivity (or reprocessing efficiency) of BLR gas: the $\mathrm{H} \beta$ responsivity is highest in the outer portions of the BLR where incident continuum fluxes are lower (Korista \& Goad 2004; Goad \& Korista 2014). An increase in ionizing continuum luminosity will therefore lead to an increase in the emissivity-weighted BLR radius; this effect is known as "breathing." For a BLR having a radial velocity gradient with the highest-velocity gas at small radii close to the black hole, breathing will preferentially boost the flux of the low-velocity line core relative to the high-velocity wings of the broad line, making the line profile narrower.

BLR breathing has usually been considered in the context of large luminosity changes over sufficiently long timescales that emission-line lags can be seen to vary between widely separated epochs of monitoring (e.g., O'Brien et al. 1995; Korista \& Goad 2004; Cackett \& Horne 2006; Bentz et al. 2007). The $\mathrm{H} \beta$ width-luminosity anticorrelation seen in our data (and in recent work by Park et al. 2012a) further illustrates that BLR breathing occurs even on the shortest observed timescales (days to weeks) in response to modest variations in the AGN continuum. While our data do not directly reveal short-timescale changes in lag or BLR radius, the relationship between $\mathrm{H} \beta$ width and luminosity implies that the BLR radius responds very rapidly to continuum flux changes. A further consequence of this finding is that the reverberation transfer function will be nonstationary even on timescales shorter than a single typical monitoring campaign. Measured transfer functions (Bentz 2010b; Grier et al. 2013a) will thus represent temporally smeared averages of intrinsically time-varying functions.

Since broad-line flux variations are driven by continuum variations, it is also of interest to examine the relationship between broad-line width and continuum flux. However, the time delay between continuum and line variations introduces substantial additional scatter to the relationship, and in our data, the broad $\mathrm{H} \beta$ widths show a much clearer relationship with $\mathrm{H} \beta$ flux than with continuum flux. The temporal mismatch between line width and continuum flux measurements can be partially alleviated by selecting continuum and emission-line measurements that are separated by the measured emission-line lag time (Park et al. 2012a). Unfortunately, the frequent weather-related gaps in our light curves do not leave enough matched pairs of data points to obtain clear results. Another possibility would be to interpolate the data to obtain matched pairs of line widths and continuum fluxes after correcting for the broad-line lag time, but we will defer that investigation in order to use the higher-cadence $V$-band photometric light curves instead of the spectroscopic continuum measurements presented here.

The behavior of broad-line widths in response to luminosity variations has important ramifications for the accuracy of single-epoch spectroscopic determinations of black hole masses. These are derived by using the AGN continuum luminosity as a proxy for the BLR radius and computing a virial product based on $\mathrm{VP}=L^{\gamma}(\Delta V)^{2} / G$, where $\gamma$ represents the slope of the radius-luminosity relationship. Recent work finds $r_{\mathrm{BLR}} \propto L_{5100}^{0.53 \pm 0.04}$ for the full sample of reverberationmapped AGNs, where $L_{5100}$ is the AGN continuum luminosity at $\lambda=5100 \AA$ (Bentz et al. 2013). The luminosity of the broad Balmer emission lines can also be used as a proxy for continuum luminosity (Greene \& Ho 2005). The accuracy of these single-epoch methods for mass estimation relies implicitly on the assumption that line width and luminosity must be anticorrelated in a given AGN, such that the derived masses will not change dramatically as the luminosity varies. Our results provide additional reassuring evidence that this is generally the case; see Denney et al. (2009a) and Park et al. (2012a) for detailed discussion of the impact of variability on single-epoch mass determinations. If $r_{\mathrm{BLR}} \propto L_{5100}^{0.5}$ and the $\mathrm{H} \beta$ luminosity scales linearly with continuum luminosity, then an AGN maintaining a constant virial product would exhibit a line width-luminosity relationship having slope $\beta=-0.25$, similar to the slopes observed in Mrk 40, Mrk 1511, and NGC 4593. However, it is important to note that this naive prediction for the slope of the $\mathrm{H} \beta$ width-luminosity relationship is an oversimplification; for example, observations of NGC 5548 show that it exhibits more complex behavior. The broad $\mathrm{H} \beta$ flux in NGC 5548 does not scale linearly with continuum flux (the "intrinsic Baldwin effect"; Gilbert \& Peterson 2003), and the relationship between BLR radius and continuum luminosity measured from long-term monitoring of NGC 5548 exhibits a steeper slope than the radius-luminosity relationship for the reverberation-mapped sample as a whole (Kilerci Eser et al. 2015).

Recently, Guo \& Gu (2014) carried out line width measurements for a sample of 60 quasars having at least six epochs of SDSS spectroscopy, and they examined the correlation between line FWHM and luminosity for C IV, $\mathrm{Mg} 2, \mathrm{H} \beta$, and $\mathrm{H} \alpha$. Interestingly, $75 \%$ of their sources show a positive correlation between broad-line width and luminosity, and only three sources exhibited an anticorrelation at the $\geqslant 90 \%$ confidence level. Another example of a positive widthluminosity correlation for the Balmer lines was recently found in PG 1613+658 (Zhang 2014), using data from the Wise Observatory quasar monitoring campaign (Kaspi et al. 2000). This behavior is very different from that seen in our sample, and is inconsistent with expectations from the scenario of BLR breathing determined by radial gradients in responsivity (with higher responsivity at larger radius) as described above. It will be interesting to examine this trend for larger samples of quasars over long time baselines as more multi-epoch spectra become available for quasars over longer time baselines. If broad-line widths in high-luminosity quasars respond to continuum variations in a manner strongly different from what we observe in our sample of Seyfert galaxies, this would potentially compromise the reliability of single-epoch black hole mass estimates derived from quasar spectra.

\section{IMPLICATIONS OF BROAD-LINE VELOCITY SHIFTS FOR BINARY BLACK HOLE SEARCHES}

The spectral decompositions make it possible to measure night-to-night changes in the broad $\mathrm{H} \beta$ velocity centroids. Our results for Mrk 40, Mrk 50, Mrk 279, Mrk 1511, and NGC 4593 illustrate that systematic shifts of $\sim 100 \mathrm{~km} \mathrm{~s}^{-1}$, correlated with flux changes, are common and occur over timescales similar to the continuum variability timescale. The largest shifts are seen for NGC 4593, in which the broad $\mathrm{H} \beta$ centroid changed by $266 \pm 11 \mathrm{~km} \mathrm{~s}^{-1}$ over a 30 -day interval, as seen in Figure 19.

Broad-line velocity shifts are expected in any AGN for which the reverberation transfer function is asymmetric about the line center, which will occur when radial motions are present in the BLR (e.g., Blandford \& McKee 1982; Capriotti et al. 1982; Antonucci \& Cohen 1983; Ulrich et al. 1984; Stirpe 
et al. 1988; Rosenblatt \& Malkan 1990). The reverberation process can be described in terms of a convolution of the continuum light curve $C(t)$ by a velocity-dependent transfer function,

$$
L\left(v_{r}, t\right)=\int_{0}^{\infty} \Psi\left(v_{r}, \tau\right) C(t-\tau) d \tau
$$

where the transfer function $\Psi(v, \tau)$ is a function of line of sight velocity $v_{r}$ and of time delay $\tau$, and $L(v, t)$ gives the resulting time-dependent emission-line profile (Blandford \& McKee 1982; Peterson 2001). If BLR clouds are in circular orbits around the black hole, as in a circular Keplerian disk, then the transfer function will show short lags at high velocities on both the blueshifted and redshifted sides of the line profile, and at small line of sight velocities there will be a broad distribution of lag time. If the BLR gas is dominated by radial motions, however, the transfer function will be strongly asymmetric about the line center. For example, in a BLR dominated by radial inflow, the redshifted gas on the near side of the BLR will have near-zero lag, while the blueshifted gas on the far side of the BLR will have the longest lags. Representative transfer functions for simple kinematic models are shown by Welsh \& Horne (1991), Bentz (2010b), Grier et al. (2013a), and Pancoast et al. (2014b).

In addition to the imprint of BLR kinematics on the transfer function, the transverse Doppler shift and gravitational redshift in the BLR will produce redward asymmetries in the transfer function, particularly in the high-velocity wings (Goad et al. 2012). The combination of transverse Doppler shift and gravitational redshift, together with any radial motions, should ensure that all transfer functions are at least modestly asymmetric in structure even if the BLR cloud motions are predominantly rotational. Absorption or scattering within the BLR may also alter the shape of $\Psi(v, \tau)$ in an asymmetric fashion.

With the best-quality reverberation-mapping data obtained over the past several years, it has become possible to examine the velocity-dependent structure of the transfer function in several low-redshift AGNs by measuring the lag as a function of velocity across the line profile (Kollatschny 2003; Bentz et al. 2008; Denney et al. 2010; Barth et al. 2011b) and by reconstructing the full two-dimensional shape of the transfer function (Bentz 2010b; Grier et al. 2013a). Dynamical modeling of reverberation data has also begun to yield constraints on the transfer function shape (Pancoast et al. 2012, 2014b) A primary result that has emerged from these investigations is that the $\mathrm{H} \beta$ transfer functions for nearby Seyfert galaxies exhibit a broad diversity of structures: some objects have nearly symmetric transfer functions consistent with expectations for a Keplerian disk, while others have strong asymmetries suggestive of inflowing or outflowing motions.

As an illustration of why an asymmetric transfer function will lead to broad-line velocity centroid changes, consider a radially inflowing BLR. If the continuum luminosity increases, the prompt red-wing response will shift the line centroid redward initially until the blue wing response from gas on the far side of the BLR is seen at a later date. Similarly, a decrease in continuum luminosity will initially shift the line centroid blueward. The opposite behavior would be seen in an outflowing BLR. Line centroid variability will depend on the continuum light curve shape and variability amplitude, the broad-line width, and the degree of asymmetry in the transfer function. For a specified transfer function, the direction of line profile shifts will depend on the recent gradient in the continuum light curve rather than on the absolute luminosity of the continuum. Either blueshifts or redshifts can occur at a given continuum flux level, depending on the recent history of continuum variations.

These considerations are relevant for binary black hole searches because one proposed method for identification of binary black holes involves searching for temporal variations in the line of sight velocity of broad emission lines in quasars. (See Dotti et al. 2012; Popović 2012, for recent reviews of binary black hole phenomenology, optical spectroscopic signatures, and search techniques). If one member of a binary black hole is active and possesses a BLR, then the orbital motion of the binary will cause the broad emission lines to oscillate in velocity over an orbital period. A substantial amount of effort has been invested recently in searching for broad-line velocity changes in quasars having two or more epochs of spectroscopic observations. One strategy is to search for objects having very large broad-line velocity offsets (i.e., $|\Delta V| \gtrsim 1000 \mathrm{~km} \mathrm{~s}^{-1}$ relative to the host galaxy), and then obtain follow-up spectroscopy to check whether the broad-line velocity drifts over time in a manner consistent with expectations for orbital motion (Tsalmantza et al. 2011; Eracleous et al. 2012; Decarli et al. 2013; Liu et al. 2014). An alternate method is to start with the general quasar population, rather than restricting to objects showing strong velocity offsets initially, and to search for changes in broad-line centroid velocity between two widely separated epochs of spectroscopic observations (Ju et al. 2013; Shen et al. 2013). The discussion in this section is primarily (but not exclusively) relevant to this latter method.

Such searches are still in their initial stages. In recent searches using SDSS data, Shen et al. (2013) examined $\mathrm{H} \beta$ velocity shifts in a sample of 1347 quasars having two or more epochs of spectroscopy, finding 28 objects with significant velocity shifts (of typically $\sim 100-300 \mathrm{~km} \mathrm{~s}^{-1}$ ) and identifying seven of these as being promising candidate binaries. Ju et al. (2013) carried out a similar search using $\mathrm{Mg}$ 2, finding seven candidate sub-pc binaries with velocity changes of $>280 \mathrm{~km} \mathrm{~s}^{-1}$ from a starting sample of 1523 quasars having spectroscopy with $\mathrm{S} / \mathrm{N}>10$ at two epochs. For these candidates, continuing long-duration follow-up spectroscopy will be required in order to rule out alternative explanations and conclusively demonstrate binary motion.

In discussion of these search efforts, it is generally recognized that broad-line velocity changes can happen as a result of structural changes in the BLR occurring over a dynamical timescale (years to decades) or longer (Shen et al. 2013), and such changes have been seen in multi-year monitoring observations of NGC 5548, for example (Wanders $\&$ Peterson 1996; Sergeev et al. 2007). The appearance or fading of hotspots in the BLR has also been suggested as a possible mechanism to induce line profile changes and apparent velocity shifts (Decarli et al. 2013). However, asymmetric reverberation seems to have been overlooked as a possible cause of the detected velocity shifts in these binary black hole searches. Our data provide a clear demonstration that broadline velocity centroids can vary significantly on short timescales solely as a result of reverberation, and do not provide direct evidence for bulk acceleration of the BLR. 
Based on our small sample of nearby, low- to moderateluminosity AGNs, it is difficult to extrapolate our results to make any useful predictions for the distribution of broad-line velocity shifts expected as a function of timescale for quasar surveys. We have carried out simple simulations using asymmetric transfer functions similar to those observed in Arp 151 (Bentz 2010b) or NGC 5548 (Pancoast et al. 2014b) and find that velocity shifts of $\gtrsim 300-400 \mathrm{~km} \mathrm{~s}^{-1}$ can occasionally occur for plausible AGN variability amplitudes. However, the range of transfer function shapes and asymmetries is completely unknown for the quasar population. For a given AGN variability model and transfer function model, it would be straightforward to predict the expected distribution of velocity changes as a function of timescale, but such an effort is beyond the scope of this paper.

Here, our goal is simply to call attention to the fact that reverberation does indeed produce significant velocity shifts on short timescales as a result of the BLR response to continuum fluctuations. This has been understood since the earliest reverberation mapping studies, and our spectral decompositions provide a way to examine broad $\mathrm{H} \beta$ velocity shifts in Seyfert 1 nuclei with high-cadence sampling over a 2.5 month baseline. Considering that we find one object showing a velocity change of $\gtrsim 250 \mathrm{~km} \mathrm{~s}^{-1}$ in our small sample over this brief monitoring duration, it seems entirely plausible that, in large AGN samples observed over long time baselines, the tail of the distribution of reverberation-induced velocity shifts could extend to substantially larger velocities than the values we observe here. It is difficult to exclude the possibility that most or all of the recently identified candidate binaries might simply be exhibiting velocity changes due to this relatively prosaic effect, rather than exhibiting acceleration due to binary orbital motion. In large samples of quasars with two-epoch spectroscopy, selection of targets with the largest velocity shifts will preferentially select objects from the tail of the distribution of large reverberation-induced velocity shifts, and we suggest that the majority of candidate binaries selected via this method will most likely be false positives.

This reverberation effect will also contribute random velocity noise or jitter that could impede the detection of genuine binaries (particularly wide-separation or nearly face-on binaries), rather analogous to the impact of stellar activity on the detectability of exoplanets via the radial-velocity method. Furthermore, the radial-velocity curve for NGC 4593 (Figure 19) illustrates another potential concern. In this case, the velocity curve could be mistaken for a near-complete cycle of sinusoidal oscillation, when in reality the line centroid is simply responding to a down-and-up variation in the continuum luminosity. In order to convincingly demonstrate binary orbital motion and rule out reverberating impostors similar to this example, it will be necessary to monitor broadline velocity variations over at least two orbital cycles, with sufficient cadence to sample the orbit well, and also to demonstrate that the velocity variations are uncorrelated with continuum flux variations. The expected orbital periods for quasars hosting supermassive black hole binaries span years to decades (e.g., Shen et al. 2013), thus confirmation of black hole binaries by this method will require a very long-term effort.

\section{CONCLUSIONS}

We present spectroscopic data, light curves, and other measurements from a 69-night reverberation mapping campaign carried out at the Lick $3 \mathrm{~m}$ telescope in Spring 2011 for a sample of 15 low-redshift Seyfert 1 galaxies. Although our observing run was hampered by worse-than-average weather, we obtained data of excellent quality for a subset of our sample, suitable for measurement of light curves for multiple emission lines. Our primary results can be summarized as follows.

1. Expanding on our team's recent work, we have carried out multicomponent decompositions of the nightly blueside spectra from our campaign. The decompositions enable measurement of light curves for blended features including $\mathrm{Fe}$ II and $\mathrm{He}$ II, and measurements of $\mathrm{H} \beta$ light curves and widths are improved by deblending $\mathrm{H} \beta$ from other contaminating emission-line and continuum components. Obtaining a time series of $\mathrm{H} \beta$ line profiles without contamination from other overlapping features is also important for measurement of velocity-resolved reverberation signals and transfer functions, and for application of dynamical modeling methods to derive black hole masses (Pancoast et al. 2014b). In Appendix C below, we demonstrate that fitting and removal of continuum components prior to constructing the rms spectrum is also necessary in order to avoid a potentially severe bias that can occur when measuring line widths from the rms spectrum. Spectral decompositions should be carried out as a routine part of reverberation mapping data analysis, provided that the $\mathrm{S} / \mathrm{N}$ of the data is sufficient for multicomponent fits to yield useful results.

2. General variability properties for our sample are consistent with trends seen in previous reverberationmapping programs, including higher responsivity for the higher-order Balmer lines, and very high responsivity for He II. While cross-correlation lags can be measured between the emission-line and continuum light curves presented here, lags measured against $V$-band photometric light curves will have better reliability as a result of the higher $\mathrm{S} / \mathrm{N}$ of the photometric data and the absence of correlated flux-calibration errors between the photometric and spectroscopic data.

3. The anticorrelation between broad-line width and flux observed in four objects demonstrates that the BLR "breathes" (i.e., the emissivity-weighted BLR radius increases as a function of AGN luminosity) even on short timescales of days to weeks in response to continuum variations. This implies that reverberation transfer functions will be nonstationary even during the course of a single monitoring campaign. This breathing effect is responsible for the relationship between broad-line profile widths measured in mean and rms spectra, as we illustrate by means of simulations in Appendix B.

4. In NGC 4593 we observe a broad $\mathrm{H} \beta$ velocity shift of $\sim 250 \mathrm{~km} \mathrm{~s}^{-1}$ over the course of one month, and other AGNs in our sample exhibit $\mathrm{H} \beta$ velocity shifts of order $100 \mathrm{~km} \mathrm{~s}^{-1}$ over similar timescales. Such velocity shifts caused by asymmetric reverberation represent an important source of confusion noise for binary black hole searches based on multi-epoch quasar spectroscopy. When only two (or a few) observations of a given 
AGN are available, line-profile centroid shifts due to velocity-dependent reverberation can mimic the expected acceleration signature of binary orbital motion, producing a spurious identification of a candidate binary. Typical velocity changes caused by asymmetric reverberation will be up to a few hundred $\mathrm{km} \mathrm{s}^{-1}$, similar to the velocity shifts detected in many of the recently identified candidate binaries (Ju et al. 2013; Shen et al. 2013). In general, reverberation will produce a velocity-jitter noise floor that could limit the detectability of binary black holes in spectroscopic searches for radial-velocity oscillations.

Additional papers in this series will present $V$-band photometric light curves, measurements of broad-line lags and velocity-resolved reverberation using both cross-correlation and JAVELIN (Zu et al. 2011) methodology, black hole virial mass estimates, and BLR dynamical modeling using the methods described by Pancoast et al. (2011) and Pancoast et al. (2014a). Following the completion of this program, our reduced and scaled spectra will be made available to the community for archival use. ${ }^{33}$

We thank the Lick Observatory staff for their tireless efforts during our observing run. We gratefully acknowledge contributions of observing time and data from Dawoo Park, Donghoon Son, Matthew Auger, Alessandro Sonnenfeld, Robert da Silva, Michele Fumagalli, Jessica Werk, Michael Gregg, Chelsea Harris, Jeffrey Lee, Liliana Lopez, Nao Suzuki, Jonathan Trump, Hassen Yesuf, Peter Nugent, David Tytler, Xavier Prochaska, Gábor Worseck, Melissa Graham, and Michael Childress. We thank Daeseong Park for many very helpful discussions about data-analysis methods, and the anonymous referee for many helpful suggestions that improved the presentation of this paper. This work has been supported by National Science Foundation (NSF) grants AST-1108835 and 1412693 (UC Irvine), 1107865 (UCSB), 1107812 and 1412315 (UCLA), and 1108665 (UC Berkeley). A.P. acknowledges support from the NSF through the Graduate Research Fellowship Program. A.V.F.'s group at UC Berkeley received additional funding through NSF grant AST-1211916, Gary \& Cynthia Bengier, the Richard \& Rhoda Goldman Fund, the TABASGO Foundation, and the Christopher R. Redlich Fund. T.T. acknowledges a Packard Research Fellowship. The work of D.S. and R.J.A. was carried out at the Jet Propulsion Laboratory, California Institute of Technology, under a contract with the National Aeronautics and Space Administration (NASA). R.J.A. was also supported by Gemini-CONICYT grant number 32120009. Research by J.L.W. was supported by NSF grant AST-1102845. J.H.W. acknowledges support by the National Research Foundation of Korea (NRF) grant funded by the Korea government (MEST; No.2012-006087). V.N.B. acknowledges assistance from NSF Research at Undergraduate Institutions (RUI) grant AST-1312296. J.M.S. is supported by an NSF Astronomy and Astrophysics Postdoctoral Fellowship under award AST-1302771. A.M.D. acknowledges support from The Grainger Foundation. Portions of the data analysis were completed during the Summer 2013 workshop "A Universe of Black Holes" at the Kavli Institute for Theoretical

\footnotetext{
33 The Lick AGN Monitoring Project 2008 spectroscopic dataset (Bentz et al. 2009b, 2010a) has been recently released for public use, and is currently available at the web site of A.J.B. at UC Irvine.
}

Physics, attended by A.J.B., T.T., and J.-H.W., and we are grateful for the hospitality of the KITP during this program. This research has made use of the NASA/IPAC Extragalactic Database (NED), which is operated by the Jet Propulsion Laboratory, California Institute of Technology, under contract with NASA. We thank Jelena Kovačević and collaborators for making their new Fe II templates available to the community. Funding for the SDSS and SDSS-II has been provided by the Alfred P. Sloan Foundation, the Participating Institutions, the NSF, the U.S. Department of Energy, NASA, the Japanese Monbukagakusho, the Max Planck Society, and the Higher Education Funding Council for England. The SDSS Web Site is http://www.sdss.org/. The SDSS is managed by the Astrophysical Research Consortium for the Participating Institutions. The Participating Institutions are the American Museum of Natural History, Astrophysical Institute Potsdam, University of Basel, University of Cambridge, Case Western Reserve University, University of Chicago, Drexel University, Fermilab, the Institute for Advanced Study, the Japan Participation Group, Johns Hopkins University, the Joint Institute for Nuclear Astrophysics, the Kavli Institute for Particle Astrophysics and Cosmology, the Korean Scientist Group, the Chinese Academy of Sciences (LAMOST), Los Alamos National Laboratory, the Max-Planck-Institute for Astronomy (MPIA), the Max-Planck-Institute for Astrophysics (MPA), New Mexico State University, Ohio State University, University of Pittsburgh, University of Portsmouth, Princeton University, the United States Naval Observatory, and the University of Washington.

Facilities: Shane (Kast)

\section{APPENDIX A ADDITIONAL SPECTRA}

During the first few clear nights of our Lick program (2011 March 29-31), we observed several additional galaxies that were initially selected as possible reverberation targets based on SDSS or other spectra or on AGN classifications from the literature, but which were subsequently discarded from our monitoring sample. Some of these objects were too faint for regular monitoring, and others appeared to be in a low flux state compared with previous observations. In one case we did not detect any emission lines in a galaxy previously classified as a Seyfert 1. Interestingly, three AGNs selected from SDSS based on their strong broad $\mathrm{H} \beta$ emission had significantly different spectral properties when observed at Lick: Mrk 474, Mrk 728, and Mrk 1494 all appeared to be excellent reverberation mapping targets based on SDSS spectra observed in 2003-2006, but by 2011 the previously strong broad $\mathrm{H} \beta$ lines faded so completely that they would be classified as Type 1.9 Seyferts. Transitions like these are not unprecedented (e.g., Tohline \& Osterbrock 1976; Aretxaga et al. 1999; Denney et al. 2014; LaMassa et al. 2014; Scott et al. 2014; Shappee et al. 2014), but little statistical information is available on the timescale or duty cycle of such dramatic changes. For completeness, we present notes on the discarded targets below, along with plots of the spectra (Figure A1) and comparisons with the earlier SDSS spectra for the three AGNs that exhibited a near-complete disappearance of broad $\mathrm{H} \beta$ (Figure A2).

$K A 1925+50$ : this Seyfert 1 galaxy at $z=0.067$ is one of the brightest AGNs in the field observed during the main Kepler mission, and initial Kepler light curves were presented by 


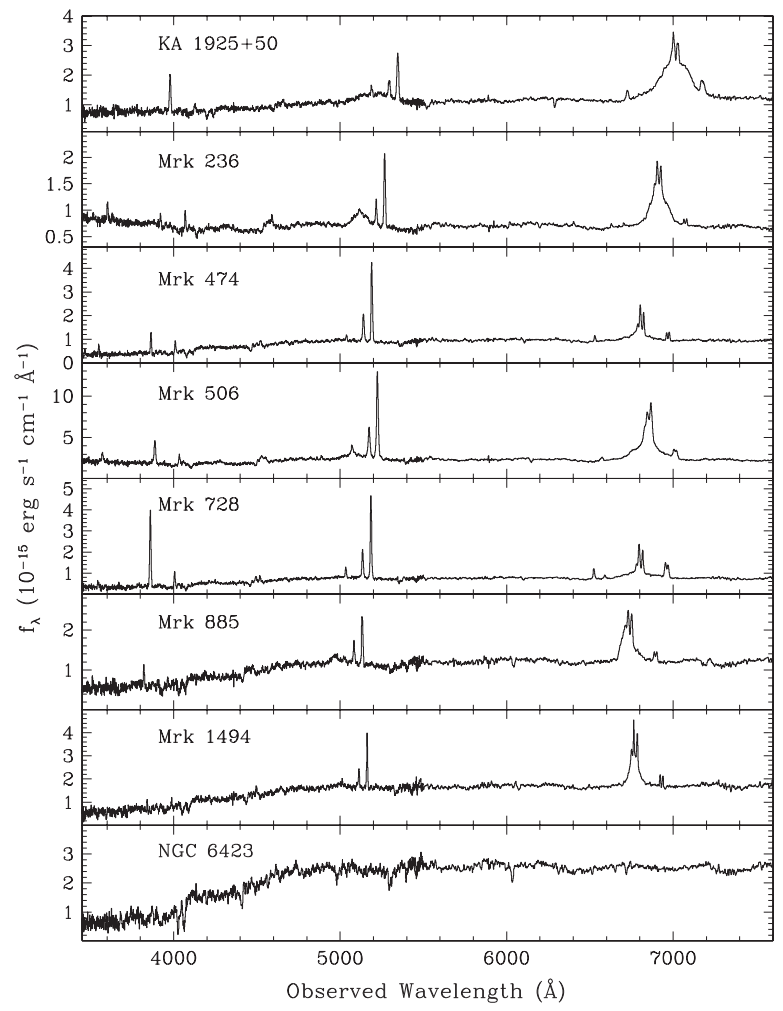

Figure A1. Kast spectra of objects observed at the start of the campaign but not selected for continued monitoring.

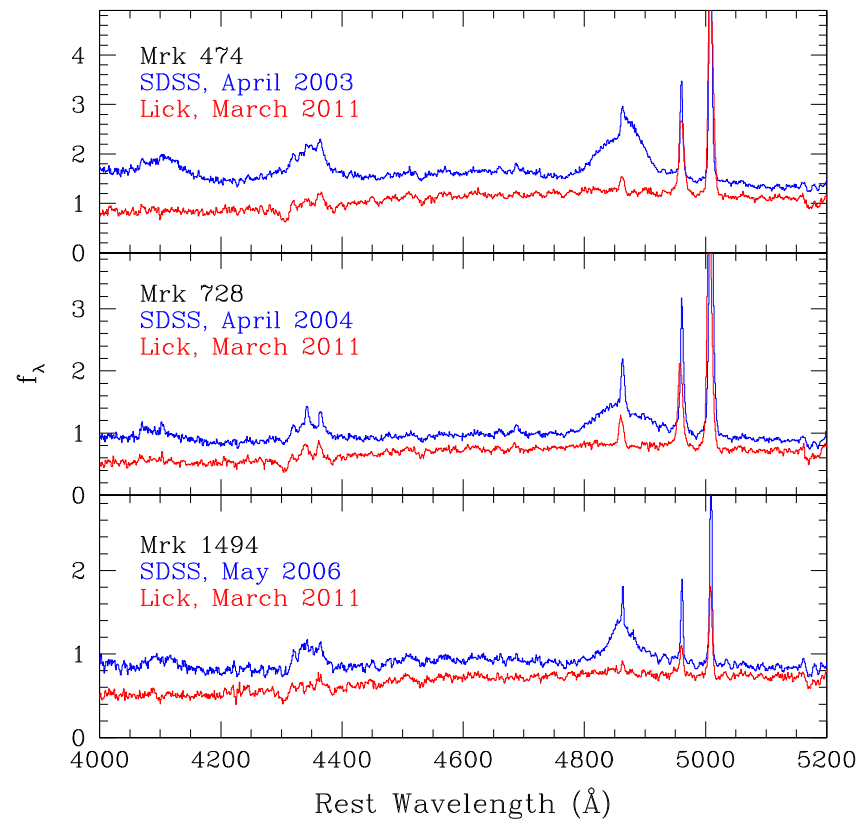

Figure A2. Comparison between SDSS and Kast spectra for Mrk 474, Mrk 728, and Mrk 1494, illustrating dramatic fading of the broad $\mathrm{H} \beta$ emission lines over a span of 5-8 yr. The Lick spectra were not taken under photometric conditions, and have been scaled by an arbitrary factor to facilitate comparison.

Mushotzky et al. 2011. We confirm its Seyfert 1 classification, but its broad $\mathrm{H} \beta$ emission line has a low amplitude relative to the host-galaxy continuum, so it is not an ideal target for $\mathrm{H} \beta$ reverberation mapping although $\mathrm{H} \alpha$ emission is very strong. Its full designation is 2MASX J19250215 + 5043134 .
See Edelson \& Malkan (2012) for further information about this object.

Mrk 236: this AGN appeared to be in a fainter state than in an earlier SDSS spectrum, and was discarded in favor of other objects in the same R.A. range.

Mrk 474 (NGC 5683): in the SDSS spectrum (observed 2003 April 4), this is a Seyfert 1 galaxy with very strong broad $\mathrm{H} \beta$ emission. Our Lick spectrum shows that the AGN has faded dramatically, and broad $\mathrm{H} \beta$ is no longer visible.

Mrk 506: the broad $\mathrm{H} \beta$ emission in this galaxy is too weak to be useful for reverberation mapping, and our spectrum appears very similar to the SDSS spectrum.

Mrk 728: this is another example of an object having strong broad $\mathrm{H} \beta$ in the SDSS spectrum (observed 2004 April 24) but showing little or no detectable broad $\mathrm{H} \beta$ in our Lick data.

Mrk 885: this object is classified in NED as a Seyfert 1.0, but has relatively weak broad $\mathrm{H} \beta$ and is too faint and starlightdominated to be a good reverberation-mapping target.

Mrk 1494: the broad $\mathrm{H} \beta$ line in this AGN also appears to have faded dramatically in comparison with the SDSS spectrum, observed on 2006 May 28. The narrow [O III] and $\mathrm{H} \beta$ lines are also significantly fainter in the Lick spectrum than in the earlier SDSS data.

NGC 6423 (Ark 524): this galaxy is listed in the VéronCetty and Véron (2006) catalog and in NED as a Type 1.5 Seyfert, but we do not detect emission lines in its spectrum.

\section{APPENDIX B \\ ON THE RELATIONSHIP BETWEEN MEAN AND RMS PROFILE WIDTHS}

Broad-line widths measured from rms spectra are commonly used to derive black hole masses from reverberation data. In light of the very widespread use of rms profile widths for determination of black hole masses and for calibrating the overall normalization of the AGN black hole mass scale (Onken et al. 2004; Peterson et al. 2004; Collin et al. 2006; Woo et al. 2010; Graham et al. 2011; Park et al. 2012b; Grier et al. 2013b; Ho \& Kim 2014) we include in the following sections a description of simulations which may be helpful in clarifying certain aspects of the behavior of the rms profile in time-series spectroscopy of AGNs.

We first examine the relationship between broad-line profile widths in mean and rms spectra. The mean line profile gives a useful representative view of the typical emission-line width during an observational campaign. However, the mean profile incorporates the complex and nonlinear response of the line profile to continuum variations (including profile width variations and centroid shifts as well as flux variations, as illustrated in Section 6.3), so it is not identical to the singleepoch profile that would be observed when the AGN is at its mean flux. The interpretation of the rms spectrum is less straightforward. It is sometimes stated that the rms spectrum represents the variable portion of an emission line, because nonvarying components do not contribute to the rms (e.g., Peterson et al. 1998; Peterson \& Wandel 1999; Fromerth \& Melia 2000). However, the BLR is not composed of distinct variable and nonvariable components; rather, different regions of the BLR have different responsivities to continuum variations, and the rms spectrum does not correspond directly to the spectrum emitted by any specific physical subcomponent of the BLR. Essentially, the rms spectrum is useful as a tool for visualization of the relative degree of variability as a function 
of wavelength across the line profile, due to spatial gradients in emission-line responsivity across the BLR combined with the effects of light-travel time across the BLR, but it is not obvious how to interpret the physical meaning of the velocity width of an rms line profile. Since reverberation-mapping campaigns are unable to acquire continuously sampled observations as an AGN varies, the detailed shape of both the mean and rms spectra depends additionally on the observational sampling pattern used during the campaign. Finally, continuum variability and noise in the data will have an impact on the line profiles and widths measured from rms spectra; we examine these effects in Appendix $\mathrm{C}$ below.

The width of broad $\mathrm{H} \beta$ is typically found to be slightly narrower in rms profiles than in mean profiles (e.g., Sergeev et al. 1999; Collin et al. 2006; Bentz et al. 2010a). Combining our sample with the previous 2008 Lick AGN Monitoring Project sample (using measurements from Park et al. 2012a based on spectral fitting methods similar to those employed here) plus additional Lick observations of Zw 229-015 (Barth et al. 2011a) and KA1858 + 4850 (Pei et al. 2014), we have 17 independent measurements of the ratio $\sigma(\mathrm{rms}) / \sigma$ (mean) for $\mathrm{H} \beta$ in 15 AGNs, including two measurements each for Mrk 40 and $\mathrm{Zw}$ 229-015. This ratio ranges from 0.43 to 1.06 , with a mean value of 0.85 and a standard deviation of 0.15 among these 17 measurements. Similarly, in the sample of 15 AGNs examined by Sergeev et al. (1999), the average value of the ratio $\sigma(\mathrm{rms}) / \sigma$ (mean) for the broad $\mathrm{H} \beta$ line is 0.87 .

The narrower widths of rms profiles for $\mathrm{H} \beta$ can be understood as a consequence of high-ionization gas in the inner BLR having a lower responsivity to continuum variations than lowionization gas more distant from the black hole; Korista \& Goad (2004) predict a factor of $\sim 5$ change in $\mathrm{H} \beta$ responsivity across the radial range of the BLR. The high-velocity wings of the line profile are emitted primarily by low-responsivity portions of the BLR, while higher-responsivity gas contributes mainly to the line core. As a result, the core of the $\mathrm{H} \beta$ profile varies more strongly than the wings, making the rms profile narrower than the mean profile.

Why is it that $\sigma(\mathrm{rms}) / \sigma$ (mean) typically takes the value $\sim 0.85$ ? The purpose of this Appendix is to illustrate by means of simple numerical simulations that this ratio is dictated by the relationship between the broad $\mathrm{H} \beta$ single-epoch luminosity and line width (as described in Section 6), which is fundamentally a consequence of the variation in emission-line responsivity across the BLR. The calculations presented here are not derived from a physical model of BLR variations; this is merely a numerical experiment designed to examine the behavior of rms profiles when the line width responds in a well-defined way to flux variations.

We begin with a Gaussian line profile as the baseline profile for steady-state emission. Using the method of Timmer and König (1995), we generate a random light curve of some specified duration, sampling, power spectrum shape, and rms variability amplitude (or $F_{\text {var }}$ ). As we describe below, the details of the light curve construction have little or no effect on the ratio of rms profile width to mean profile width.

Then, we generate a time series of line profiles in which both the line luminosity and its width vary in response to continuum fluctuations. Assuming a delta function with lag $\tau$ for the velocity-integrated transfer function, at each time $t$ the integrated luminosity $L$ of the emission line is made to scale linearly with the input continuum light curve luminosity at time

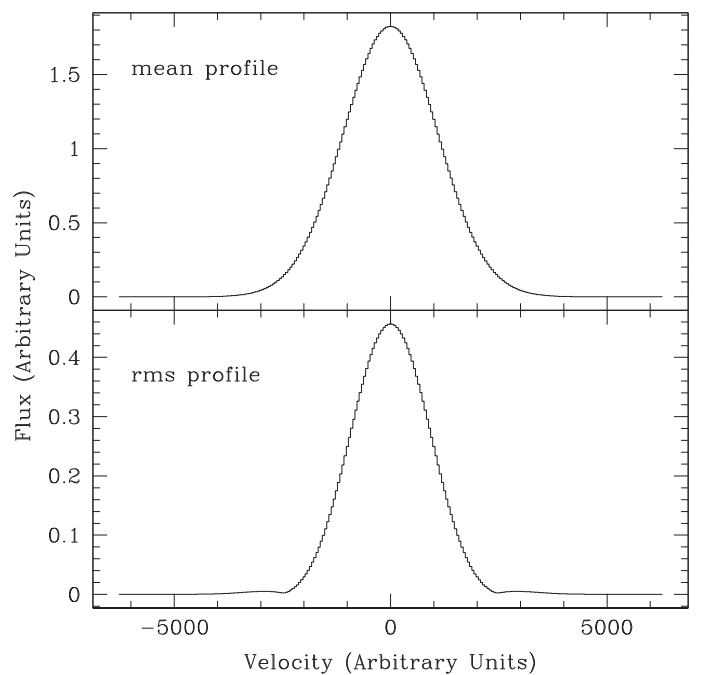

Figure A3. Example from a simulation of a mean and rms line profile, constructed from a time series of Gaussian profiles having variable luminosities and widths as described in the text, with $\sigma^{2} L^{0.5}=$ constant. The amplitude of the rms profile is set by the overall variability amplitude of the input light curve in the simulation, but its shape is nearly independent of the light curve properties.

$t-\tau$. The width of the Gaussian profile is also made to vary, such that the product $\sigma^{2} L^{0.5}$ remains constant as the line luminosity varies. This represents the most common behavior seen in our sample [i.e., $\sigma(\mathrm{H} \beta) \propto f(\mathrm{H} \beta)^{-0.25}$ ], and corresponds to a situation in which the single-epoch virial product remains roughly constant as the AGN luminosity varies. From the time series of resultant line profiles, we construct the mean and rms profiles and calculate $\sigma_{\text {line }}$ for each.

The result of this experiment is surprisingly simple. In the mean spectrum, the emission line has a profile that is very nearly Gaussian. As expected for a broad line showing higher variability amplitude in the core than in the wings, the rms profile is narrower than the mean profile. The rms profile has an approximately Gaussian shape, but exhibits additional structure in the faint high-velocity wings, and this profile shape is nearly independent of the input light curve properties. The peak height of the rms profile depends on the variability amplitude of the light curve, while its width is only very weakly dependent on variability amplitude. In the limit of small but nonzero light curve variance, the ratio $\sigma(\mathrm{rms}) / \sigma$ (mean) converges to 0.824 . As the light curve variance is increased, we find that this ratio becomes very slightly sensitive to the input light curve properties. For example, when the input light curve is scaled to have $F_{\mathrm{var}}=0.2$, the simulations produce a result of $\sigma(\mathrm{rms}) / \sigma($ mean $)=0.83 \pm 0.01$, where the uncertainty represents the standard deviation of values from 1000 simulations carried out with different random light curves. Figure A3 shows an example of a mean and rms profile obtained from one such simulation with $F_{\text {var }}=0.2$ over a light curve having 500 equally spaced points, with a fluctuation power spectrum of the form $P(\nu) \propto \nu^{-2.7}$ (where $\nu$ is the temporal frequency of fluctuations). The rms profile shape would appear virtually identical for different choices of light curve duration or sampling, power spectrum slope, or variability amplitude, although its absolute normalization depends on $F_{\mathrm{var}}$.

These simulations produce a ratio $\sigma(\mathrm{rms}) / \sigma$ (mean) that is remarkably close to the observed mean ratio of $0.85 \pm 0.15$ 
found from our Lick Observatory monitoring campaigns. In other words, the typical ratio of line widths observed in rms and mean line profiles simply reflects the fact that in most AGNs, the broad $\mathrm{H} \beta$ line maintains a roughly constant value of the product $L^{0.5} \sigma^{2}$ as the AGN luminosity varies. The larger dispersion of values observed in real AGNs most likely results from the range of different (non-Gaussian) profile shapes in different AGNs, and also differences in the scaling relationship between single-epoch luminosity and profile width. Changing the power-law relationship between line width and luminosity does alter the width of the rms profile relative to the mean profile. For example, if we calculate models for a flatter singleepoch scaling relationship of $\sigma_{\text {line }} \propto L^{-0.07}$ (as observed in Mrk $50)$, the simulations yield $\sigma(\mathrm{rms}) / \sigma($ mean $)=0.92 \pm 0.01$.

The prevailing use of rms profile widths as the basis for calibrating the AGN black hole mass scale underscores the need for further work to understand the rms spectrum by means of of detailed simulations of BLR line profiles incorporating BLR dynamics and photoionization physics, including luminosity-dependent changes in emission-line responsivity (Korista \& Goad 2004; Goad \& Korista 2014). The dynamical modeling approach for interpretation of reverberation-mapping data (Pancoast et al. 2012, 2014a; Li et al. 2013) avoids the problem of relying on simplistic measures of line width from either mean or rms spectra, and can provide more direct constraints on black hole masses. For future development of these models, it should be a high priority to incorporate a spatially variable and luminosity-dependent emission-line responsivity based on photoionization modeling of BLR structure, in order to more accurately model an observed time series of variable line profiles. If the responsivity is assumed to be spatially and/or temporally constant across the BLR, it will not generally be possible for these models to replicate the observed anticorrelation between line width and luminosity, or the relationship between mean and rms profile widths.

\section{APPENDIX C BIASES IN RMS PROFILE WIDTHS}

Since line widths measured from rms spectra are used to derive black hole masses, it is useful to examine possible sources of systematic error in these measurements. The rms spectrum blends information about emission-line variations, continuum variations, and random noise in the data, and it can deviate substantially from the "ideal" rms spectrum that would be derived if a single broad emission line could be observed without contamination from other spectral components or noise. In this appendix, we describe simulations designed to examine the impact of continuum variability and photoncounting noise on line widths measured from rms spectra.

\section{C.1 Bias due to Continuum Variability}

This same simulation methodology described above allows us to examine a potential source of bias in the rms profile width that can occur when the rms spectrum is constructed from a set of spectra that have not had the variable AGN continuum removed. Components of constant flux, such as narrow emission lines or starlight, do not contribute to the rms spectrum except to the extent that they show some night-tonight variations due to seeing differences, miscentering of the AGN in the slit, random wavelength or flux calibration errors, or Poisson noise. However, it is not widely appreciated that the variable AGN continuum underlying an emission line can affect the shape of the rms profile of the line, and that this can potentially introduce significant biases into black hole virial masses determined from rms profile widths.

The reason this bias occurs is that the continuum and emission-line variations are out of phase with one another: there will be portions of the light curves where the continuum is rising while the emission-line flux is falling, and vice versa. This out-of-phase variability alters the rms amplitude of the total (line plus continuum) flux, and tends to preferentially suppress the rms flux in the line profile wings relative to the line core. Consequently, the rms profile constructed from totalflux spectra can sometimes have a significantly different width than it would if the emission line could be observed in isolation without continuum contamination. Even a modest bias in determining the rms profile width can have a substantial and deleterious effect on the derivation of black hole masses, because black hole mass scales as the square of the line width. The typically employed procedure of fitting and subtracting the continuum from the total-flux rms spectrum prior to measuring the profile width does not alleviate this problem.

To quantify this bias, we carried out simulations using Gaussian line profiles as described above. As before, the total line luminosity responds linearly to continuum variations, and the line luminosity and width vary such that $\sigma^{2} L^{0.5}$ remains constant. For the input light curves, we assumed a fluctuation power spectrum of the form $P(\nu) \propto \nu^{-2.7}$, similar to the properties of nearby AGNs monitored by the Kepler mission (Mushotzky et al. 2011), and we carried out simulations with light curves of varying duration and various values of $F_{\text {var }}$. The velocity-integrated transfer function was assumed to be a delta function at a lag of 10 days. The emission line was set to have a mean equivalent width of $120 \AA$ relative to the mean AGN continuum flux density (typical for the broad $\mathrm{H} \beta$ lines in our sample) and an initial width of $\sigma_{\text {line }}=2000 \mathrm{~km} \mathrm{~s}^{-1}$, and the continuum flux level was assumed to be flat over the wavelength range of interest. Spectra were constructed for velocity bins of $60 \mathrm{~km} \mathrm{~s}^{-1}$, corresponding closely to the $1 \AA$ wavelength bins of the Kast blue-side data. These simulations do not include the effects of photon-counting noise, random wavelength or flux calibration errors, contributions of narrow-line or host-galaxy components to the spectra, or nonuniform sampling cadence, all of which can further alter the rms spectrum. (The impact of photon-counting noise is described in the next section.)

Starting from each simulated continuum light curve, we generated a time series of emission-line profiles. At each time step, we created a spectrum of the emission line alone, and a total-flux spectrum including both the line and the continuum. Two versions of the rms spectrum were then computed from the time series of line-only and total-flux spectra. Then, the line dispersion $\sigma_{\text {line }}$ was measured from each of these. For the totalflux rms spectrum, the surrounding continuum level was removed prior to measuring the line dispersion by subtracting the minimum value of the rms spectrum. In order to minimize the impact of rms flux in the very distant high-velocity line wings on the $\sigma_{\text {line }}$ measurements, the dispersion was measured only over the wavelength range between the two minima on either side of the profile, in cases where the rms profile exhibited local minima straddling the line profile. Similarly, for the line-only rms spectrum we also measured the dispersion only over the wavelength range between the two local minima 

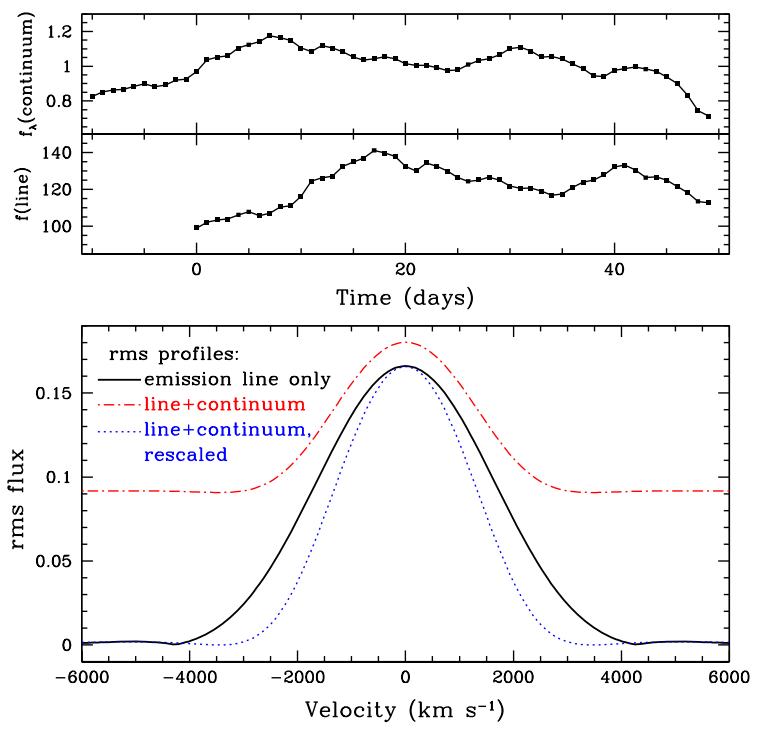

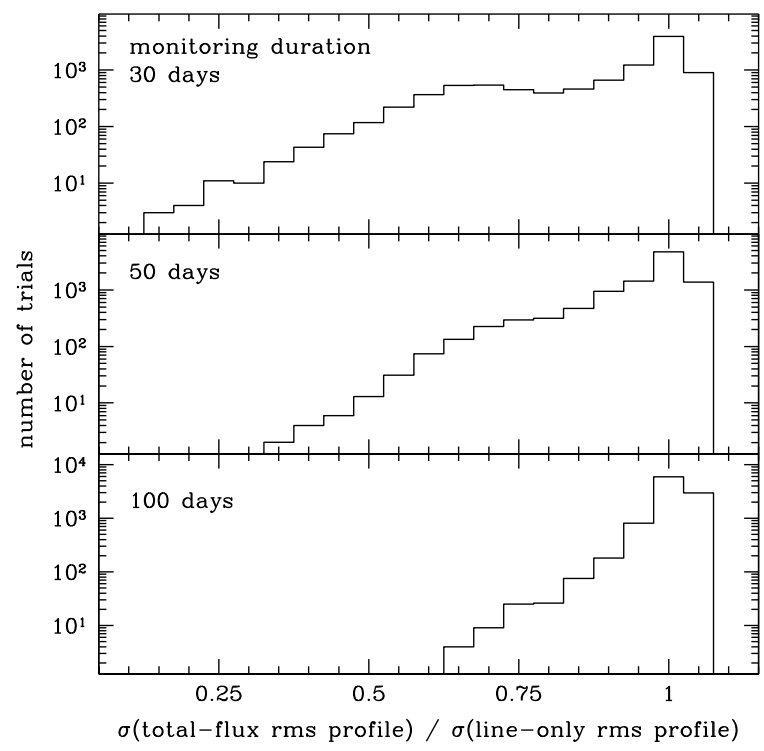

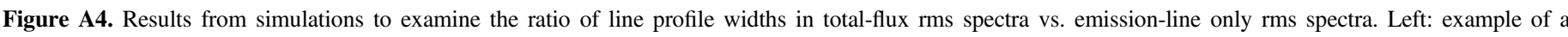

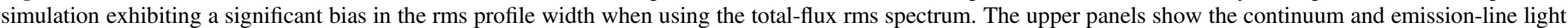

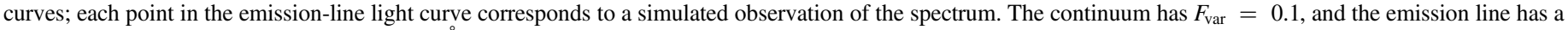

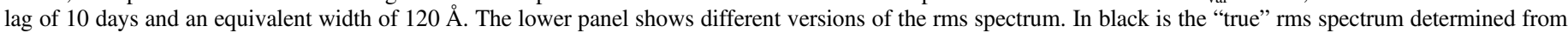

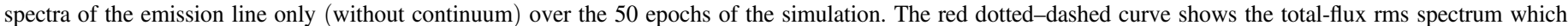

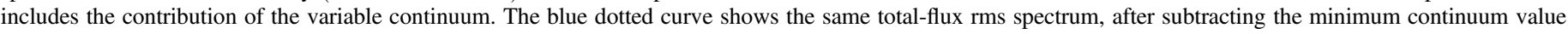

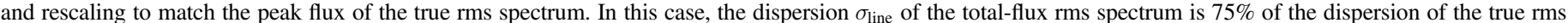

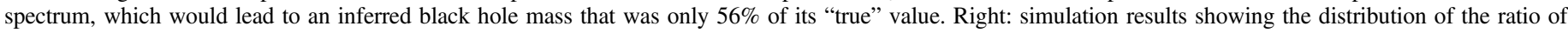

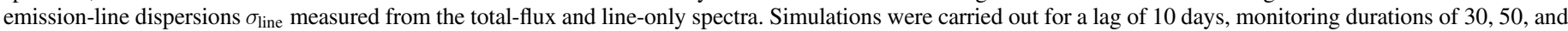
100 days with daily sampling, and $F_{\mathrm{var}}=0.1$.

that occur in the profile wings. In real data, these very shallow minima would most likely not be visible as a result of the additional contributions to the continuum level of the rms spectra from other noise sources, and the rms profile width would be measured over some visually defined wavelength range corresponding roughly to the full width at zero intensity of the rms profile.

The simulations show that the width of the total-flux rms line profile can often deviate very substantially from the width of the line-only rms profile, particularly when the monitoring duration is only a few times longer than the emission-line lag. Figure A4 (left panel) shows an example of one simulation, with a 50 day spectroscopic monitoring duration (i.e., 5 times longer than the lag, and assuming nightly observations with no gaps). The figure illustrates both the line-only rms profile, which should be considered the "true" rms profile for this simulation, and the total-flux (line plus continuum) rms profile. By subtracting the continuum level from the total-flux rms spectrum and rescaling it to match the peak of the true rms profile, it is immediately apparent that the total-flux rms profile is much narrower than the true profile. In this instance, $\sigma($ total $) /$ $\sigma($ true $)=0.75$, which would bias the inferred black hole mass by a factor of $0.75^{2}=0.56$.

To examine the distribution of this bias factor, we carried out a set of these simulations for campaigns of duration 30,50, and 100 days, assuming nightly sampling in each case and a fixed value of $F_{\mathrm{var}}=0.1$ for the AGN continuum. In Figure A4 (right panel), we plot the distribution of the ratio $\sigma$ (total $) / \sigma$ (true) determined from a set of $10^{4}$ simulations at each campaign duration. The bias distribution is a strong function of campaign duration. For example, we find that the percentage of simulations returning $\sigma$ (total) $/ \sigma$ (true) $<0.75$ is 22,6 , and $0.3 \%$ for campaign durations of 30,50 , and 100 days, respectively. In most of the recent large reverberation-mapping programs carried out by our team and others, the monitoring duration is usually at least $\sim 5$ times longer than the measured $\mathrm{H} \beta$ lags of the targets, but as future programs push toward higher-luminosity AGNs at higher redshifts there may be increasing numbers of AGNs for which the spectroscopic time series is sampled less intensively, over a monitoring duration that might be just barely sufficient to measure the lag (typically about $\sim 3$ times the lag). In that situation, the total-flux rms spectrum can frequently give a misleading measure of the emission-line width.

In actual reverberation data sets, the magnitude of this bias will depend on the details of the light curve shape and variability amplitude, the transfer function shape, the profile shape and equivalent width of the emission line, and the sampling cadence of the observations, so the outcome will be different for every time series of data for every AGN. Our intention is not to carry out exhaustive simulations for a broad range of AGN parameters, but simply to demonstrate that this bias can occur under typical circumstances of a reverberation measurement when the total-flux rms spectrum is used. The simulation results shown in Figure A4 illustrate that $\sigma_{\text {line }}$ can sometimes be severely underestimated even when the light curves have sufficient duration and sampling that the emissionline lag could easily be measured accurately.

We propose that the best way to avoid this problem is to carry out continuum fitting and subtraction prior to construction of the rms spectrum, so that line widths can be measured from an rms spectrum that is free of continuum variability contamination. Other advantages of carrying out spectral decompositions for measurement of $\mathrm{H} \beta \mathrm{rms}$ profile widths have been discussed by Park et al. (2012a); they focused on the importance of removing He II, Fe II, narrow-line components, 

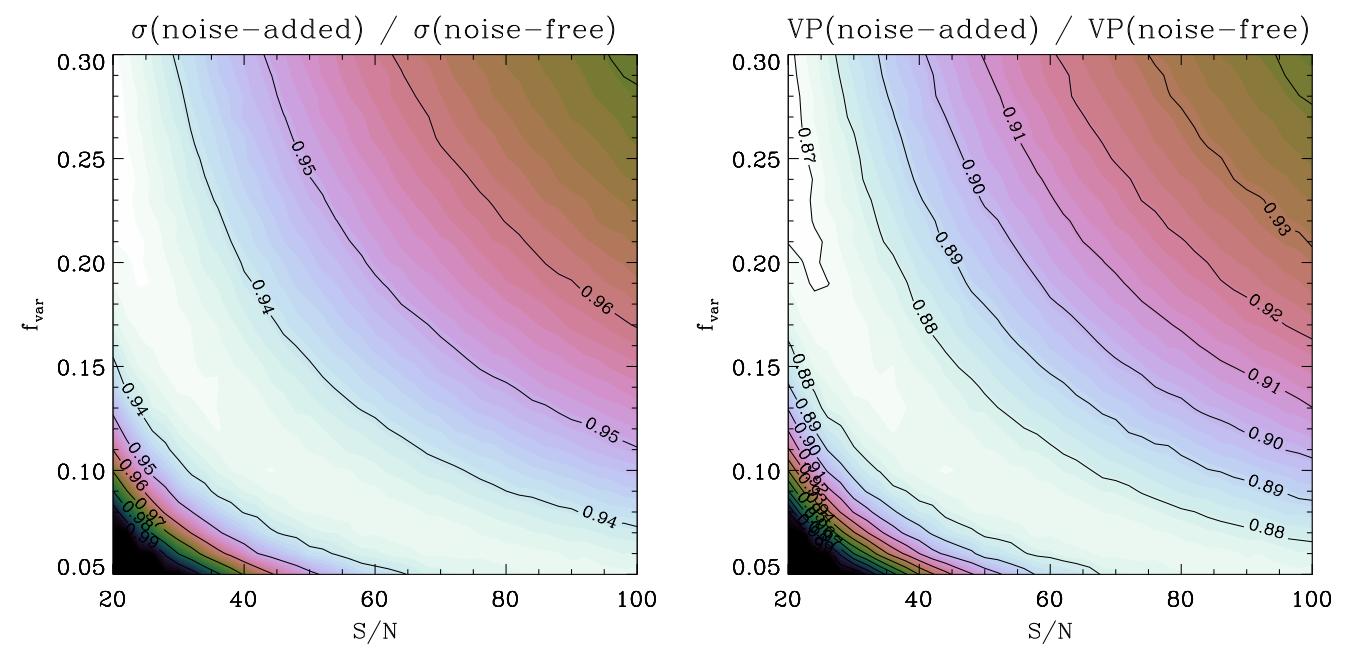

Figure A5. Results of simulations testing the impact of $\mathrm{S} / \mathrm{N}$ on line widths measured from the rms spectrum. Tests were carried out for a range of values of $\mathrm{S} / \mathrm{N}$ and $F_{\mathrm{var}}$ as indicated on the $x$ and $y$ axes. Contour levels depict the median result from 1000 simulations done at each value of $\mathrm{S} / \mathrm{N}$ and $F_{\mathrm{var}}$. Left panel: Ratio of the line dispersion of the rms profile including noise contributions, to the line dispersion of the rms profile constructed from noise-free spectra. Right panel: Ratio of the virial product (VP) for the noise-added and noise-free cases, where the virial product is proportional to the square of the line dispersion. The lower left corner of each panel, at $F_{\mathrm{var}}<0.1$ and low values of $\mathrm{S} / \mathrm{N}$, corresponds to reverberation measurements that would most likely fail to detect a lag.

and starlight prior to constructing rms spectra, but they did not remove the nonstellar featureless continuum. Figure 6 illustrates the construction of an rms spectrum for the broad $\mathrm{H} \beta$ line in isolation, with other emission-line and continuum components removed, and in this paper we have measured the profile widths from rms spectra constructed in this way. It is still necessary to carry out a local continuum subtraction by fitting a linear continuum model to the rms flux on either side of the $\mathrm{H} \beta$ line in order to remove the small residual rms flux level due to random noise in the data. For the case of Mrk 40 illustrated in Figure 6, the values of $\sigma_{\text {line }}$ for the total-flux rms spectrum and the line-only rms spectrum differ by just $2 \%$. Based on our simulations, only a very small difference would be expected given that the campaign duration was $\sim 20$ times longer than the $\mathrm{H} \beta$ lag of $\sim 4$ days for this AGN (Bentz et al. 2009b).

\section{C.2 Bias Due to Photon Statistics}

The finite $\mathrm{S} / \mathrm{N}$ of spectroscopic observations is the source of a different bias that can afflict profile widths in rms spectra. The pixel values in an rms spectrum are determined not just by variability in the AGN, but also by the noise level in the spectra. A nonvarying object would still be observed to have a nonzero rms spectrum due to photon-counting noise and detector noise. The contribution of these noise sources can affect the profile width in the rms spectrum simply because the flux density is largest in the core of the emission line, hence the noise level will be highest there as well. The larger contribution of noise in the line core will elevate the peak of the line profile in the rms spectrum relative to the wings, making the line slightly narrower than it would appear in the absence of noise. While this is not a large effect, its impact is amplified because virial masses scale as the square of the line width.

To examine the impact of $\mathrm{S} / \mathrm{N}$ on rms profile widths, we carried out further simulations using the same methodology described above. Continuum light curves and the resulting time series of line profiles were created using the same prescriptions and parameters described in the previous section. The continuum and emission-line spectra for each simulation epoch were added to give the total flux spectrum at each epoch. Then, Gaussian random noise was added to each spectrum in the time series. The Gaussian dispersion for this pixel-to-pixel noise level was set by fixing the continuum $\mathrm{S} / \mathrm{N}$ to a given value, and scaling the noise level by $S_{i}^{1 / 2}$, where $S_{i}$ is the flux density at pixel $i$ in the spectrum. Each simulation was run for a total spectroscopic monitoring duration of 100 days with nightly sampling, an emission-line lag of 10 days, and a specified value of $F_{\text {var }}$ for the continuum variability amplitude.

From each simulation, we computed a "true" rms spectrum from the time series of noise-free emission-line spectra only, with no continuum contributions. Using the noise-added data, we subtracted the continuum flux level from each nightly spectrum and constructed a line-only rms spectrum that included the noise contribution. The line dispersion of the true rms profile was measured as described previously, by including the flux only between the two local minima on either side of the rms profile. To measure the width of the noise-added rms profile, we fit and subtracted the flat noise continuum using a straight-line fit, and then calculated the line dispersion over the same velocity range that was used for the true rms profile measurement. The ratio of the noise-added rms line width to the true rms line width gives the bias factor, $\sigma$ (noise-added $) / \sigma$ (noise-free). Since the continuum flux was subtracted from each noise-added spectrum prior to constructing the noiseadded rms spectrum, this measurement is immune to the continuum-variability bias described in the previous section.

The degree of bias in the rms profile width depends primarily on $F_{\mathrm{var}}$ and on the $\mathrm{S} / \mathrm{N}$ of the data. We carried out simulations for continuum $\mathrm{S} / \mathrm{N}$ ranging from 20 to 100 (in increments of 2) and for $F_{\text {var }}$ ranging from 0.05 to 0.30 (in increments of 0.01 ). For each set of these parameters, we carried out 1000 simulation trials and determined the median bias factor. Figure A5 displays the results, illustrating the ratio $\sigma$ (noiseadded $) / \sigma$ (noise-free) as a function of $F_{\text {var }}$ and the continuum S/ $\mathrm{N}$. We also display the bias in the virial product, $\mathrm{VP}$ (noiseadded $) / \mathrm{VP}($ noise-free $)=[\sigma(\text { noise-added }) / \sigma(\text { noise-free })]^{2}$. For typical parameters of a successful reverberation measurement, corresponding to $F_{\mathrm{var}} \gtrsim 0.1$ and $\mathrm{S} / \mathrm{N}$ of 30-100 $\mathrm{pixel}^{-1}$, the rms 
profile width is biased low by $\sim 3-7 \%$, and virial products are biased low by $\sim 6-13 \%$. The bias vanishes in the limit of high $\mathrm{S} / \mathrm{N}$ and high variability amplitude, but even at $\mathrm{S} / \mathrm{N}=100$ per pixel the virial products are biased low by $\sim 5-10 \%$. Unlike the continuum-variability bias described in the previous section, this effect is not alleviated by having a long and well-sampled campaign with a large number of observations. For given values of $\mathrm{S} / \mathrm{N}$ and $F_{\mathrm{var}}$, increasing the number of spectra in the time series will not remove this systematic effect of photoncounting noise on the rms profile width, although it will make the rms spectrum less noisy.

Given the data quality for typical reverberation campaigns, these simulations suggest that virial products calculated using rms spectrum profile widths will be systematically low typically by $\sim 10 \%$. The standard practice of normalizing reverberation-based black hole masses to the local $M_{\mathrm{BH}}-\sigma$ relation of inactive galaxies (Onken et al. 2004; Woo et al. 2010) will compensate for this offset, and consequently the the virial $f$-factor derived from this procedure (e.g., Onken et al. 2004; Woo et al. 2010) will be biased high by 10\%. The impact of this effect is much smaller than the overall uncertainty on the mean $f$ factor for the set of reverberationmapped AGNs, so this does not currently represent a major problem for derivation of AGN black hole masses, but nevertheless it is a real effect that is present in all reverberation-mapping data. Park et al. (2012a) presented a maximum-likelihood method to calculate rms spectra that was designed to be immune to this problem; further tests of this or other alternative methods to calculate rms spectra would be worthwhile.

\section{REFERENCES}

Abazajian, K. N., Adelman-McCarthy, J. K., Agüeros, M. A., et al. 2009, ApJS, 182, 543

Antonucci, R. R. J., \& Cohen, R. D. 1983, ApJ, 271, 564

Aretxaga, I., Joguet, B., Kunth, D., Melnick, J., \& Terlevich, R. J. 1999, ApJL, 519, L123

Barth, A. J., Nguyen, M. L., Malkan, M. A., et al. 2011, ApJ, 732, 121

Barth, A. J., Pancoast, A., Thorman, S. J., et al. 2011, ApJL, 743, L4

Barth, A. J., Pancoast, A., Bennert, V. N., et al. 2013, ApJ, 769, 128

Bentz, M. C., Denney, K. D., Cackett, E. M., et al. 2007, ApJ, 662, 205

Bentz, M. C., Walsh, J. L., Barth, A. J., et al. 2008, ApJL, 689, L21

Bentz, M. C., Peterson, B. M., Netzer, H., Pogge, R. W., \& Vestergaard, M. 2009, ApJ, 697, 160

Bentz, M. C., Walsh, J. L., Barth, A. J., et al. 2009, ApJ, 705, 199

Bentz, M. C., Walsh, J. L., Barth, A. J., et al. 2010, ApJ, 716, 993

Bentz, M. C., et al. 2010, ApJL, 720, L46

Bentz, M. C., Denney, K. D., Grier, C. J., et al. 2013, ApJ, 767, 149

Bian, W.-H., Huang, K., Hu, C., et al. 2010, ApJ, 718, 460

Blandford, R. D., \& McKee, C. F. 1982, ApJ, 255, 419

Boroson, T. A., \& Green, R. F. 1992, ApJS, 80, 109

Brewer, B. J., Treu, T., Pancoast, A., et al. 2011, ApJL, 733, L33

Bruzual, G., \& Charlot, S. 2003, MNRAS, 344, 1000

Cackett, E. M., \& Horne, K. 2006, MNRAS, 365, 1180

Capriotti, E. R., Foltz, C. B., \& Peterson, B. M. 1982, ApJ, 261, 35

Cardelli, J. A., Clayton, G. C., \& Mathis, J. S. 1989, ApJ, 345, 245

Carini, M. T., \& Ryle, W. T. 2012, ApJ, 749, 70

Collin, S., Kawaguchi, T., Peterson, B. M., \& Vestergaard, M. 2006, A\&A, 456, 75

Decarli, R., Dotti, M., Fumagalli, M., et al. 2013, MNRAS, 433, 1492

Denney, K. D., Bentz, M. C., Peterson, B. M., et al. 2006, ApJ, 653, 152

Denney, K. D., Peterson, B. M., Dietrich, M., Vestergaard, M., \& Bentz, M. C. 2009, ApJ, 692, 246

Denney, K. D., Peterson, B. M., Pogge, R. W., et al. 2009, ApJL, 704, L80

Denney, K. D., Peterson, B. M., Pogge, R. W., et al. 2010, ApJ, 721, 715

Denney, K. D., de Rosa, G., Croxall, K., et al. 2014, ApJ, 796, 134

Dietrich, M., Kollatschny, W., Alloin, D., et al. 1994, A\&A, 284, 33
Dietrich, M., Appenzeller, I., Vestergaard, M., \& Wagner, S. J. 2002, ApJ, 564,581

Dotti, M., Sesana, A., \& Decarli, R. 2012, AdAst, 2012, 940568

Edelson, R., Turner, T. J., Pounds, K., et al. 2002, ApJ, 568, 610

Edelson, R., \& Malkan, M. 2012, ApJ, 751, 52

Edelson, R., Vaughan, S., Malkan, M., et al. 2014, arXiv:1409.1613

Eracleous, M., Boroson, T. A., Halpern, J. P., \& Liu, J. 2012, ApJS, 201, 23 Filippenko, A. V. 1982, PASP, 94, 715

Foley, R. J., Challis, P. J., Chornock, R., et al. 2013, ApJ, 767, 57

Fromerth, M. J., \& Melia, F. 2000, ApJ, 533, 172

Gilbert, K. M., \& Peterson, B. M. 2003, ApJ, 587, 123

Goad, M. R., Korista, K. T., \& Ruff, A. J. 2012, MNRAS, 426, 3086

Goad, M. R., \& Korista, K. T. 2014, MNRAS, 444, 43

Graham, A. W., Onken, C. A., Athanassoula, E., \& Combes, F. 2011, MNRAS, 412, 2211

Greene, J. E., \& Ho, L. C. 2005, ApJ, 630, 122

Greene, J. E., \& Ho, L. C. 2007, ApJ, 670, 92

Grier, C. J., Peterson, B. M., Horne, K., et al. 2013, ApJ, 764, 47

Grier, C. J., Martini, P., Watson, L. C., et al. 2013, ApJ, 773, 90

Guo, H., \& Gu, M. 2014, ApJ, 792, 33

Hillenbrand, L. A., Miller, A. A., Covey, K. R., et al. 2013, AJ, 145, 59

Hinshaw, G., Larson, D., Komatsu, E., et al. 2013, ApJS, 208, 19

Ho, L. C., \& Kim, M. 2014, ApJ, 789, 17

Horne, K. 1986, PASP, 98, 609

Horne, K., Peterson, B. M., Collier, S. J., \& Netzer, H. 2004, PASP, 116, 465

Ju, W., Greene, J. E., Rafikov, R. R., Bickerton, S. J., \& Badenes, C. 2013, ApJ, 777, 44

Kaspi, S., Smith, P. S., Netzer, H., et al. 2000, ApJ, 533, 631

Kelly, B. C. 2007, ApJ, 665, 1489

Kilerci Eser, E., Vestergaard, M., Peterson, B. M., Denney, K. D., \& Bentz, M. C. 2015, ApJ, 801, 8

Kollatschny, W. 2003, A\&A, 407, 461

Korista, K. T., \& Goad, M. R. 2004, ApJ, 606, 749

Kovačević, J., Popović, L. Č., \& Dimitrijević, M. S. 2010, ApJS, 189, 15

Krolik, J. H. 2001, ApJ, 551, 72

LaMassa, S. M., Cales, S., Moran, E. C., et al. 2014, arXiv:1412.2136

Li, Y.-R., Wang, J.-M., Ho, L. C., Du, P., \& Bai, J.-M. 2013, ApJ, 779, 110

Liu, X., Shen, Y., Bian, F., Loeb, A., \& Tremaine, S. 2014, ApJ, 789, 140

Maoz, D., Netzer, H., Leibowitz, E., et al. 1990, ApJ, 351, 75

Markwardt, C. B. 2009, adass XVIII, 411, 251

Matheson, T., Filippenko, A. V., Ho, L. C., Barth, A. J., \& Leonard, D. C. 2000, AJ, 120, 1499

Miller, J. S., \& Stone, R. P. S. 1993, Lick Obs. Tech. Rep. 66 (Santa Cruz, CA: Lick Observatory)

Mushotzky, R. F., Edelson, R., Baumgartner, W., \& Gandhi, P. 2011, ApJL, 743, L12

O’Brien, P. T., Goad, M. R., \& Gondhalekar, P. M. 1995, MNRAS, 275, 1125

Onken, C. A., Peterson, B. M., Dietrich, M., Robinson, A., \& Salamanca, I. M. 2003, ApJ, 585, 121

Onken, C. A., Ferrarese, L., Merritt, D., et al. 2004, ApJ, 615, 645

Pancoast, A., Brewer, B. J., \& Treu, T. 2011, ApJ, 730, 139

Pancoast, A., Brewer, B. J., Treu, T., et al. 2012, ApJ, 754, 49

Pancoast, A., Brewer, B. J., \& Treu, T. 2014, arXiv:1407.2941

Pancoast, A., Brewer, B. J., Treu, T., et al. 2014, MNRAS, 445, 3073

Park, D., Woo, J.-H., Treu, T., et al. 2012, ApJ, 747, 30

Park, D., Kelly, B. C., Woo, J.-H., \& Treu, T. 2012, ApJS, 203, 6

Pei, L., Barth, A. J., Aldering, G. S., et al. 2014, ApJ, 795, 38

Peterson, B. M., \& Ferland, G. J. 1986, Natur, 324, 345

Peterson, B. M., Wanders, I., Bertram, R., et al. 1998, ApJ, 501, 82

Peterson, B. M., \& Wandel, A. 1999, ApJL, 521, L95

Peterson, B. M. 2001, Advanced Lectures on the Starburst-AGN Connection, ed. I. Aretxaga, D. Kunth, \& R. Mújica (Singapore: World Scientific), 3

Peterson, B. M., Ferrarese, L., Gilbert, K. M., et al. 2004, ApJ, 613, 682

Peterson, B. M., Denney, K. D., de Rosa, G., et al. 2013, ApJ, 779, 109

Popović, L. Č. 2012, NewAR, 56, 74

Richards, G. T., Kruczek, N. E., Gallagher, S. C., et al. 2011, AJ, 141, 167

Rodríguez-Pascual, P. M., Alloin, D., Clavel, J., et al. 1997, ApJS, 110, 9

Rosenblatt, E. I., \& Malkan, M. A. 1990, ApJ, 350, 132

Santos-Lleó, M., Clavel, J., Schulz, B., et al. 2001, A\&A, 369, 57

Schlafly, E. F., \& Finkbeiner, D. P. 2011, ApJ, 737, 103

Scott, B., Bennert, V., Komossa, S., et al. 2014, BAAS, 223, 250.16

Sergeev, S. G., Pronik, V. I., Sergeeva, E. A., \& Malkov, Y. F. 1999, AJ, 118,2658

Sergeev, S. G., Doroshenko, V. T., Dzyuba, S. A., et al. 2007, ApJ, 668, 708 Seyfert, C. K. 1943, ApJ, 97, 28

Shapovalova, A. I., Popović, L. Č., Burenkov, A. N., et al. 2012, ApJS, 202, 10 
Shappee, B. J., Prieto, J. L., Grupe, D., et al. 2014, ApJ, 788, 48

Shen, Y., Greene, J. E., Strauss, M. A., Richards, G. T., \& Schneider, D. P. 2008, ApJ, 680, 169

Shen, Y. 2013, BASI, 41, 61

Shen, Y., Liu, X., Loeb, A., \& Tremaine, S. 2013, ApJ, 775, 49

Silverman, J. M., Ganeshalingam, M., \& Filippenko, A. V. 2013, MNRAS, 430, 1030

Smith, N., Silverman, J. M., Filippenko, A. V., et al. 2012, AJ, 143, 17

Stirpe, G. M., de Bruyn, A. G., \& van Groningen, E. 1988, A\&A, 200, 9

Timmer, J., \& König, M. 1995, A\&A, 300, 707

Tohline, J. E., \& Osterbrock, D. E. 1976, ApJL, 210, L117

Tsalmantza, P., Decarli, R., Dotti, M., \& Hogg, D. W. 2011, ApJ, 738, 20

Ulrich, M. H., Boksenberg, A., Bromage, G. E., et al. 1984, MNRAS, 206, 221

van der Marel, R. P., \& Franx, M. 1993, ApJ, 407, 525

van Dokkum, P. G. 2001, PASP, 113, 1420 van Groningen, E., \& Wanders, I. 1992, PASP, 104, 700

Véron, P., Gonçalves, A. C., \& Véron-Cetty, M.-P. 2002, A\&A, 384, 826

Véron-Cetty, M.-P., Joly, M., \& Véron, P. 2004, A\&A, 417, 515

Véron-Cetty, M.-P., \& Véron, P. 2006, A\&A, 455, 773

Vestergaard, M., \& Peterson, B. M. 2005, ApJ, 625, 688

Wade, R. A., \& Horne, K. 1988, ApJ, 324, 411

Wanders, I., \& Peterson, B. M. 1996, ApJ, 466, 174

Wang, J.-M., Du, P., Hu, C., et al. 2014, arXiv:1408.2337

Welsh, W. F., \& Horne, K. 1991, ApJ, 379, 586

Wills, B. J., Netzer, H., \& Wills, D. 1985, ApJ, 288, 94

Woo, J.-H., Treu, T., Malkan, M. A., \& Blandford, R. D. 2006, ApJ, 645, 900

Woo, J.-H., Treu, T., Barth, A. J., et al. 2010, ApJ, 716, 269

Wright, E. L. 2006, PASP, 118, 1711

Zhang, X. 2014, arXiv: 1410.7537

Zu, Y., Kochanek, C. S., \& Peterson, B. M. 2011, ApJ, 735, 80 